\title{
Visible-light-initiated Cross-Dehydrogenative Coupling of Quinoxalin-2(1H)-ones and Simple Amides with Air as An Oxidant
}

Long-Yong Xie ${ }^{\mathrm{a}}$, Jia-Li Hu ${ }^{\mathrm{a}}$, Yan-Xi Song ${ }^{\mathrm{a}}$, Guo-Kai Jia ${ }^{\mathrm{a}}$, Ying-Wu Lin ${ }^{\mathrm{c}}$, Jun-Yi He ${ }^{\mathrm{b}}$, Zhong $\mathrm{Cao}^{\mathrm{b}}$ and Wei-Min $\mathrm{He}^{\mathrm{a} *}$

${ }^{a}$ Department of Chemistry, Hunan University of Science and Engineering, Yongzhou, Yangzi Tang Road No. 130, 425100, China

${ }^{b}$ Hunan Provincial Key Laboratory of Materials Protection for Electric Power and Transportation, Changsha University of Science and Technology, Changsha, Wanjiali Road No. 960, 410114, China

${ }^{c}$ School of Chemistry and Chemical Engineering, University of South China, HengYang, Changshen Road No.28, 421001, China

weiminhe2016@yeah.net

Number of pages: 43

Number of figures: 64

Number of schemes: 2

Table of Content

General information S2

Experimental Section

Characterization data of products

References

${ }^{1} \mathrm{H}$ and ${ }^{13} \mathrm{C}$ NMR spectra of products 


\section{General Information}

Unless otherwise specified, all reagents and solvents were obtained from commercial suppliers and used without further purification. ${ }^{1} \mathrm{H}$ NMR spectra were recorded at $400 \mathrm{MHz}$ and ${ }^{13} \mathrm{C}$ NMR spectra were recorded at $100 \mathrm{MHz}$ by using a Bruker Avance 400 spectrometer. Chemical shifts were calibrated using residual undeuterated solvent as an internal reference $\left({ }^{1} \mathrm{H}\right.$ NMR: $\mathrm{CDCl}_{3}$ 7.26 ppm, ${ }^{13} \mathrm{C}$ NMR: $\left.\mathrm{CDCl}_{3} 77.0 \mathrm{ppm}\right)$, the chemical shifts $(\delta)$ were expressed in ppm and $\mathrm{J}$ values were given in Hz. The following abbreviations were used to describe peak splitting patterns when appropriate: $\mathrm{s}=$ singlet, $\mathrm{d}=$ doublet, $\mathrm{t}=$ triplet, $\mathrm{q}=$ quartet, $\mathrm{m}=$ multiplet, $\mathrm{dd}=$ doublet of doublets. Mass spectra were performed on a spectrometer operating on ESI-TOF. Column chromatography was performed on silica gel (200-300 mesh). Light irradiation device: Xi'an WATTECS experimental equipment co. LTD; Model: WP-VLH-1020.

\section{Experimental Section}

Typical Procedure for the Synthesis of $\mathrm{N}$-acylated 3-aminoquinoxalin-2(1H)-ones

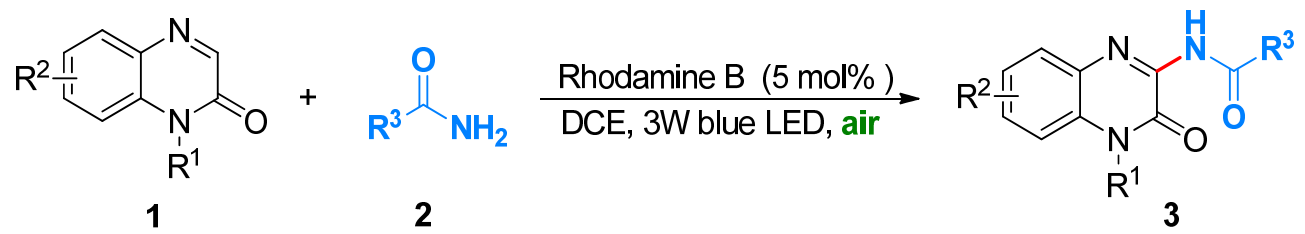

\section{Scheme S1}

To a solution of quinoxalin-2(1H)-ones $1(0.3 \mathrm{mmol})$ and amides $2(0.6 \mathrm{mmol})$ in 1,2-dichloroethane $(1.5 \mathrm{~mL})$ was added Rhodamine B $(7.2 \mathrm{mg}, 0.015 \mathrm{mmol})$. The reaction mixture was open to the air and stirred at room temperature under the irradiation of $3 \mathrm{~W}$ blue LED lamps, The progress of the reaction was monitored by TLC. The reaction typically took within 24 hours, After completion, the resulting mixture was extracted with EtOAc $(3 \mathrm{~mL} \times 3)$ and the solvent was then removed under vacuum. The residue was purified by flash column chromatography using a mixture of petroleum ether and ethyl acetate as eluent to give the desired products $\mathbf{3}$.

\section{Gram-scale synthesis of 3aa}

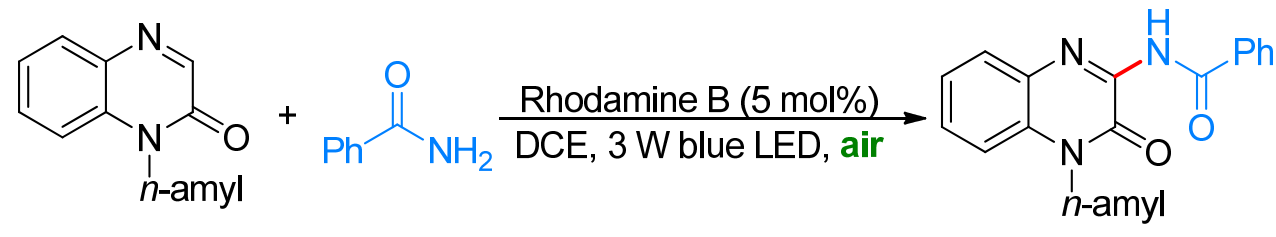

$1 \mathrm{a}, 1.08 \mathrm{~g}, 5 \mathrm{mmol}$

3aa, $83 \%, 1.39 \mathrm{~g}$ 


\section{Scheme S2}

To a solution of 1-pentylquinoxalin-2(1H)-one 1a (1.08 g, 5 mmol) and benzamide $2 \mathrm{a}(1.21 \mathrm{~g}, 10$ mmol) in 1,2-dichloroethane (40 mL) was added Rhodamine B (192 mg, $0.4 \mathrm{mmol})$. The reaction mixture was open to the air and stirred at room temperature under the irradiation of $3 \mathrm{~W}$ blue LED lamps for about $30 \mathrm{~h}$. After completion, the DCE was recovered through distillation $(94 \%$ recovery rate) and the resulting residue was extracted with EtOAc $(30 \mathrm{~mL} \times 3)$ and the solvent was then removed under vacuum. The residue was purified by flash column chromatography using a mixture of petroleum ether and ethyl acetate as eluent to give 1.39 gram of 3aa, yield $83 \%$. Performing the large-scale reaction of $\mathbf{1 a}$ in the recovered DCE provided the 3aa in $82 \%$ yield.

\section{Characterization data of products}

$N$-(3-oxo-4-pentyl-3,4-dihydroquinoxalin-2-yl)benzamide (3aa) ${ }^{1}$<smiles>CCCCCn1c(=O)c(NC(=O)c2ccccc2)nc2ccccc21</smiles>

${ }^{1} \mathrm{H}$ NMR $\left(400 \mathrm{MHz}, \mathrm{CDCl}_{3}\right): \delta=9.96(\mathrm{~s}, 1 \mathrm{H}), 8.01-7.95(\mathrm{~m}, 3 \mathrm{H}), 7.61-7.58(\mathrm{~m}, 1 \mathrm{H}), 7.53-7.46(\mathrm{~m}, 3 \mathrm{H})$, $7.39-7.31$ (m, 2 H), 4.32 (t, $J=7.6 \mathrm{~Hz}, 2 \mathrm{H}), 1.85-1.77$ (m,2 H), 1.49 - 1.38 (m, 4 H), 0.93 (t, J=6.8 Hz, $3 \mathrm{H}$ ); ${ }^{13} \mathrm{C}$ NMR $\left(100 \mathrm{MHz}, \mathrm{CDCl}_{3}\right): \delta=164.2,151.1,144.3,133.9,132.6,132.4,129.9,129.7,128.9,128.3,127.5$, 124.5, 113.6, 43.2, 29.0, 27.0, 22.3, 13.9; HRMS (ESI) $\mathrm{m} / \mathrm{z}$ calcd. for $\mathrm{C}_{20} \mathrm{H}_{22} \mathrm{~N}_{3} \mathrm{O}_{2}[\mathrm{M}+\mathrm{H}]^{+}$: 336.1707, found 336.1706 .

$N$-(4-methyl-3-oxo-3,4-dihydroquinoxalin-2-yl)benzamide (3ba) ${ }^{1}$<smiles>Cn1c(=O)c(NC(=O)c2ccccc2)nc2ccccc21</smiles>

${ }^{1} \mathrm{H}$ NMR (400 MHz, $\left.\mathrm{CDCl}_{3}\right): \delta=9.94(\mathrm{~s}, 1 \mathrm{H}), 8.00-7.98(\mathrm{~m}, 2 \mathrm{H}), 7.54-7.37$ (m, $\left.6 \mathrm{H}\right), 7.32\left(\mathrm{dd}, J_{1}=8.4 \mathrm{~Hz}, J_{2}\right.$ $=1.2 \mathrm{~Hz}, 1 \mathrm{H}), 3.79(\mathrm{~s}, 3 \mathrm{H}) ;{ }^{13} \mathrm{C} \mathrm{NMR}\left(100 \mathrm{MHz}, \mathrm{CDCl}_{3}\right): \delta=164.2,151.4,144.3,132.7,131.9,129.4,128.9$, $128.6,128.4,127.5,127.3,124.7,113.6,29.9$.

$\mathrm{N}$-(4-ethyl-3-oxo-3,4-dihydroquinoxalin-2-yl)benzamide (3ca)<smiles>CCn1c(=O)c(NC(=O)c2ccccc2)nc2ccccc21</smiles>

${ }^{1} \mathrm{H}$ NMR $\left(400 \mathrm{MHz}, \mathrm{CDCl}_{3}\right): \delta=9.95(\mathrm{~s}, 1 \mathrm{H}), 8.02-7.96(\mathrm{~m}, 3 \mathrm{H}), 7.62-7.58(\mathrm{~m}, 1 \mathrm{H}), 7.54-7.48(\mathrm{~m}, 3 \mathrm{H})$, 
$7.41-7.34$ (m, $2 \mathrm{H}), 4.41(\mathrm{q}, J=7.2 \mathrm{~Hz}, 2 \mathrm{H}), 1.43(\mathrm{t}, J=7.2 \mathrm{~Hz}, 3 \mathrm{H}) ;{ }^{13} \mathrm{C} \mathrm{NMR}\left(100 \mathrm{MHz}, \mathrm{CDCl}_{3}\right): \delta=164.2$, $150.9,144.3,133.9,132.7,132.5,129.8,129.7,128.9,128.4,127.5,124.5,113.5,38.2,12.4$.

$\mathrm{N}$-(4-benzyl-3-oxo-3,4-dihydroquinoxalin-2-yl)benzamide (3da) ${ }^{1}$<smiles>O=C(Nc1nc2ccccc2n(Cc2ccccc2)c1=O)c1ccccc1</smiles>

${ }^{1} \mathrm{H}$ NMR $\left(400 \mathrm{MHz}, \mathrm{CDCl}_{3}\right): \delta=9.97(\mathrm{~s}, 1 \mathrm{H}), 8.02-7.95(\mathrm{~m}, 2 \mathrm{H}), 7.97-7.95(\mathrm{~m}, 1 \mathrm{H}), 7.60-7.57(\mathrm{~m}, 1 \mathrm{H})$, $7.53-7.49$ (m, $2 \mathrm{H}), 7.37-7.23(\mathrm{~m}, 8 \mathrm{H}), 5.56$ (s, $2 \mathrm{H}) ;{ }^{13} \mathrm{C} \mathrm{NMR}\left(100 \mathrm{MHz}, \mathrm{CDCl}_{3}\right): \delta=164.3,151.6,144.4$, $134.5,133.8,132.7,132.4,130.2,129.6,129.1,128.9,128.4,128.0,127.6,126.7,124.7,114.4,46.7$.

$\mathrm{N}$-(4-(4-methoxybenzyl)-3-oxo-3,4-dihydroquinoxalin-2-yl)benzamide (3ea)<smiles>COc1ccc(Cn2c(=O)c(NC(=O)c3ccccc3)nc3ccccc32)cc1</smiles>

${ }^{1} \mathrm{H}$ NMR $\left(400 \mathrm{MHz}, \mathrm{CDCl}_{3}\right): \delta=9.99(\mathrm{~s}, 1 \mathrm{H}), 8.02-8.00(\mathrm{~m}, 2 \mathrm{H}), 7.97-7.95(\mathrm{~m}, 1 \mathrm{H}), 7.62-7.59(\mathrm{~m}, 1 \mathrm{H})$, $7.54-7.51$ (m, $2 \mathrm{H}), 7.40-7.32$ (m, $3 \mathrm{H}), 7.23-7.20$ (m, $2 \mathrm{H}), 6.87-6.84(\mathrm{~m}, 2 \mathrm{H}), 5.50(\mathrm{~s}, 2 \mathrm{H}), 3.76(\mathrm{~s}, 3 \mathrm{H})$; ${ }^{13} \mathrm{C} \mathrm{NMR}\left(100 \mathrm{MHz}, \mathrm{CDCl}_{3}\right): \delta=164.3,159.2,151.6,144.4,133.8,132.7,132.4,130.2,129.6,128.9,128.4$, 128.3, 127.6, 126.6, 124.7, 114.4, 55.3, 46.3; HRMS (ESI) m/z calcd. for $\mathrm{C}_{23} \mathrm{H}_{20} \mathrm{~N}_{3} \mathrm{O}_{3}[\mathrm{M}+\mathrm{H}]^{+}: 386.1499$, found 386.1504 .

ethyl 2-(3-benzamido-2-oxoquinoxalin-1(2H)-yl)acetate (3fa)<smiles>CCOC(=O)Cn1c(=O)c(NC(=O)c2ccccc2)nc2ccccc21</smiles>

${ }^{1} \mathrm{H}$ NMR $\left(400 \mathrm{MHz}, \mathrm{CDCl}_{3}\right): \delta=9.85(\mathrm{~s}, 1 \mathrm{H}), 7.80-7.97(\mathrm{~m}, 3 \mathrm{H}), 7.62-7.59(\mathrm{~m}, 1 \mathrm{H}), 7.54-7.37(\mathrm{~m}, 4 \mathrm{H})$, $7.10\left(\mathrm{dd}, J_{1}=8.4 \mathrm{~Hz}, J_{2}=0.8 \mathrm{~Hz}, 1 \mathrm{H}\right), 5.09(\mathrm{~s}, 2 \mathrm{H}), 4.26(\mathrm{q}, J=7.2 \mathrm{~Hz}, 2 \mathrm{H}), 1.27(\mathrm{t}, J=7.2 \mathrm{~Hz}, 3 \mathrm{H}) ;{ }^{13} \mathrm{C} \mathrm{NMR}$ $\left(100 \mathrm{MHz}, \mathrm{CDCl}_{3}\right): \delta=166.5,164.2,151.3,144.1,133.8,132.7,132.2,130.0,129.8,128.9,128.6,127.5,125.0$, 113.0, 62.3, 44.3, 14.1; HRMS (ESI) $\mathrm{m} / \mathrm{z}$ calcd. for $\mathrm{C}_{19} \mathrm{H}_{18} \mathrm{~N}_{3} \mathrm{O}_{4}[\mathrm{M}+\mathrm{H}]^{+}: 352.1292$, found 352.1288 .

tert-butyl 2-(3-benzamido-2-oxoquinoxalin-1(2H)-yl)acetate (3ga)<smiles>CCCCOC(=O)Cn1c(=O)c(NC(=O)c2ccccc2)nc2ccccc21</smiles> 
${ }^{1} \mathrm{H}$ NMR $\left(400 \mathrm{MHz}, \mathrm{CDCl}_{3}\right): \delta=9.87(\mathrm{~s}, 1 \mathrm{H}), 8.00-7.96(\mathrm{~m}, 3 \mathrm{H}), 7.62-7.58(\mathrm{~m}, 1 \mathrm{H}), 7.54-7.44(\mathrm{~m}, 3 \mathrm{H})$, $7.41-7.37(\mathrm{~m}, 1 \mathrm{H}), 7.10\left(\mathrm{dd}, J_{1}=8.4 \mathrm{~Hz}, J_{2}=1.2 \mathrm{~Hz}, 1 \mathrm{H}\right), 5.00(\mathrm{~s}, 2 \mathrm{H}), 1.46(\mathrm{~s}, 9 \mathrm{H}) ;{ }^{13} \mathrm{C} \mathrm{NMR}(100 \mathrm{MHz}$, $\left.\mathrm{CDCl}_{3}\right): \delta=165.5,164.2,151.3,144.1,133.8,132.7,132.2,130.1,129.8,128.9,128.5,127.5,124.9,113.0,83.6$, 45.0, 27.9; HRMS (ESI) m/z calcd. for $\mathrm{C}_{21} \mathrm{H}_{22} \mathrm{~N}_{3} \mathrm{O}_{4}[\mathrm{M}+\mathrm{H}]^{+}: 380.1605$, found 380.1062 .

$\mathrm{N}$-(4-allyl-3-oxo-3,4-dihydroquinoxalin-2-yl)benzamide (3ha)<smiles>C=CCn1c(=O)c(NC(=O)c2ccccc2)nc2ccccc21</smiles>

${ }^{1} \mathrm{H}$ NMR $\left(400 \mathrm{MHz}, \mathrm{CDCl}_{3}\right): \delta=9.93(\mathrm{~s}, 1 \mathrm{H}), 8.02-7.95(\mathrm{~m}, 3 \mathrm{H}), 7.62-7.58(\mathrm{~m}, 1 \mathrm{H}), 7.54-7.44(\mathrm{~m}, 3 \mathrm{H})$, $7.40-7.36$ (m, $1 \mathrm{H}), 7.31-7.29$ (m, $1 \mathrm{H}), 6.01-5.92(\mathrm{~m}, 1 \mathrm{H}), 5.32-5.17(\mathrm{~m}, 1 \mathrm{H}), 5.21-5.17(\mathrm{~m}, 1 \mathrm{H}), 4.99-$ 4.97 (m, $2 \mathrm{H}) ;{ }^{13} \mathrm{C}$ NMR (100 MHz, $\left.\mathrm{CDCl}_{3}\right): \delta=164.2,151.1,144.3,133.8,132.7,132.3,130.0,129.9,129.6$, 128.9, 128.3, 127.5, 124.7, 118.4, 114.2, 45.3; HRMS (ESI) $\mathrm{m} / \mathrm{z}$ calcd. for $\mathrm{C}_{18} \mathrm{H}_{16} \mathrm{~N}_{3} \mathrm{O}_{2}[\mathrm{M}+\mathrm{H}]^{+}: 306.1237$, found 306.1229 .

N-(3-oxo-4-(prop-2-yn-1-yl)-3,4-dihydroquinoxalin-2-yl)benzamide (3ia)<smiles>C#CCn1c(=O)c(NC(=O)c2ccccc2)nc2ccccc21</smiles>

${ }^{1} \mathrm{H}$ NMR $\left(400 \mathrm{MHz}, \mathrm{CDCl}_{3}\right): \delta=9.87(\mathrm{~s}, 1 \mathrm{H}), 8.00-7.96(\mathrm{~m}, 3 \mathrm{H}), 7.62-7.59(\mathrm{~m}, 1 \mathrm{H}), 7.54-7.39(\mathrm{~m}, 5 \mathrm{H})$, $5.12(\mathrm{~d}, J=2.4 \mathrm{~Hz}, 2 \mathrm{H}), 2.35(\mathrm{t}, J=2.4 \mathrm{~Hz}, 1 \mathrm{H}) ;{ }^{13} \mathrm{C} \mathrm{NMR}\left(100 \mathrm{MHz}, \mathrm{CDCl}_{3}\right): \delta=164.2,150.7,144.0,133.8$, $132.7,132.3,129.6,129.3,128.9,128.5,127.5,125.0,114.1,76.0,73.8,32.4 ;$ HRMS (ESI) m/z calcd. for $\mathrm{C}_{18} \mathrm{H}_{14} \mathrm{~N}_{3} \mathrm{O}_{2}[\mathrm{M}+\mathrm{H}]^{+}:$304.1081, found 304.1077.

N-(3-oxo-4-phenyl-3,4-dihydroquinoxalin-2-yl)benzamide (3ja)<smiles>O=C(Nc1nc2ccccc2n(-c2ccccc2)c1=O)c1ccccc1</smiles>

${ }^{1} \mathrm{H}$ NMR (400 MHz, $\left.\mathrm{CDCl}_{3}\right): \delta=9.95(\mathrm{~s}, 1 \mathrm{H}), 8.01-7.98(\mathrm{~m}, 3 \mathrm{H}), 7.68-7.57(\mathrm{~m}, 4 \mathrm{H}), 7.53-7.49(\mathrm{~m}, 2 \mathrm{H})$, $7.39-7.34(\mathrm{~m}, 3 \mathrm{H}), 7.31-7.27(\mathrm{~m}, 1 \mathrm{H}), 6.70\left(\mathrm{dd}, J_{1}=8.4 \mathrm{~Hz}, J_{2}=1.2 \mathrm{~Hz}, 1 \mathrm{H}\right) ;{ }^{13} \mathrm{C} \mathrm{NMR}\left(100 \mathrm{MHz}, \mathrm{CDCl}_{3}\right): \delta$ $=164.2,151.4,144.7,135.3,133.8,132.7,132.1,131.8,130.4,129.9,129.1,128.9,128.1,127.9,127.5,124.9$, 115.5; HRMS (ESI) $\mathrm{m} / \mathrm{z}$ calcd. for $\mathrm{C}_{21} \mathrm{H}_{16} \mathrm{~N}_{3} \mathrm{O}_{2}[\mathrm{M}+\mathrm{H}]^{+}: 342.1237$, found 342.1232 .

$\mathrm{N}$-(7-fluoro-4-methyl-3-oxo-3,4-dihydroquinoxalin-2-yl)benzamide (3ka) 
<smiles>Cn1c(=O)c(NC(=O)c2ccccc2)nc2cc(F)ccc21</smiles>

${ }^{1} \mathrm{H}$ NMR (400 MHz, $\mathrm{CDCl}_{3}$ ): $\delta=9.95(\mathrm{~s}, 1 \mathrm{H}), 7.99-7.97$ (m, $\left.2 \mathrm{H}\right), 7.65-7.58(\mathrm{~m}, 2 \mathrm{H}), 7.54-7.50$ (m, $\left.2 \mathrm{H}\right)$, $7.29-7.19$ (m, $2 \mathrm{H}), 3.78$ (s, $3 \mathrm{H}) ;{ }^{13} \mathrm{C} \mathrm{NMR}\left(100 \mathrm{MHz}, \mathrm{CDCl}_{3}\right): \delta=164.1,159.4\left(J_{\mathrm{C}-\mathrm{F}}=242.8 \mathrm{~Hz}\right), 151.0,145.2$, 133.6, $133.1\left(J_{\mathrm{C}-\mathrm{F}}=11.7 \mathrm{~Hz}\right), 128.9,127.6,127.4\left(J_{\mathrm{C}-\mathrm{F}}=2.2 \mathrm{~Hz}\right), 115.9\left(J_{\mathrm{C}-\mathrm{F}}=23.4 \mathrm{~Hz}\right), 115.0\left(J_{\mathrm{C}-\mathrm{F}}=22.6 \mathrm{~Hz}\right)$, 114.7, 114.6; ${ }^{19} \mathrm{~F}$ NMR (376 MHz, $\mathrm{CDCl}_{3}$ ): $\delta=-117.4$; HRMS (ESI) $\mathrm{m} / \mathrm{z}$ calcd. for $\mathrm{C}_{16} \mathrm{H}_{13} \mathrm{FN}_{3} \mathrm{O}_{2}[\mathrm{M}+\mathrm{H}]^{+}$: 298.0986, found 298.0983 .

$\mathrm{N}$-(7-chloro-4-methyl-3-oxo-3,4-dihydroquinoxalin-2-yl)benzamide (3la) ${ }^{1}$<smiles>Cn1c(=O)c(NC(=O)c2ccccc2)nc2cc(Cl)ccc21</smiles>

${ }^{1} \mathrm{H}$ NMR (400 MHz, $\left.\mathrm{CDCl}_{3}\right): \delta=9.91(\mathrm{~s}, 1 \mathrm{H}), 7.98-7.96(\mathrm{~m}, 2 \mathrm{H}), 7.91(\mathrm{~d}, J=2.4 \mathrm{~Hz}, 1 \mathrm{H}), 7.62-7.58(\mathrm{~m}, 1$ H), $7.53-7.50(\mathrm{~m}, 2 \mathrm{H}), 7.40\left(\mathrm{dd}, J_{1}=8.8 \mathrm{~Hz}, J_{2}=2.8 \mathrm{~Hz}, 1 \mathrm{H}\right), 7.22(\mathrm{~d}, J=8.8 \mathrm{~Hz}, 1 \mathrm{H}), 3.76(\mathrm{~s}, 3 \mathrm{H}) ;{ }^{13} \mathrm{C} \mathrm{NMR}$ $\left(100 \mathrm{MHz}, \mathrm{CDCl}_{3}\right): \delta=164.1,151.1,145.1,133.6,132.8,132.8,130.0,129.5,128.9,128.6,128.2,127.5,114.7$, 30.1 .

$\mathrm{N}$-(7-bromo-4-methyl-3-oxo-3,4-dihydroquinoxalin-2-yl)benzamide (3ma) ${ }^{1}$<smiles>Cn1c(=O)c(NC(=O)c2ccccc2)nc2cc(Br)ccc21</smiles>

${ }^{1} \mathrm{H}$ NMR $\left(400 \mathrm{MHz}, \mathrm{CDCl}_{3}\right): \delta=9.92(\mathrm{~s}, 1 \mathrm{H}), 8.10(\mathrm{~d}, J=2.4 \mathrm{~Hz}, 1 \mathrm{H}), 7.99-7.97(\mathrm{~m}, 2 \mathrm{H}), 7.63-7.51(\mathrm{~m}, 4$ $\mathrm{H}), 7.18(\mathrm{~d}, J=8.8 \mathrm{~Hz}, 1 \mathrm{H}), 3.77(\mathrm{~s}, 3 \mathrm{H}) ;{ }^{13} \mathrm{C} \mathrm{NMR}\left(100 \mathrm{MHz}, \mathrm{CDCl}_{3}\right): \delta=164.1,151.1,145.0,133.6,133.1$, 132.8, 131.7, 131.0, 129.9, 129.0, 127.6, 117.4, 115.0, 30.1 .

$\mathrm{N}$-(4-methyl-3-oxo-7-(trifluoromethyl)-3,4-dihydroquinoxalin-2-yl)benzamide (3na)<smiles>Cn1c(=O)c(NC(=O)c2ccccc2)nc2cc(C(F)(F)F)ccc21</smiles>

${ }^{1} \mathrm{H}$ NMR $\left(400 \mathrm{MHz}, \mathrm{CDCl}_{3}\right): \delta=9.84(\mathrm{~s}, 1 \mathrm{H}), 8.15(\mathrm{~d}, J=1.2 \mathrm{~Hz}, 1 \mathrm{H}), 7.92-7.89(\mathrm{~m}, 2 \mathrm{H}), 7.61\left(\mathrm{dd}, J_{1}=8.8\right.$ $\left.\mathrm{Hz}, J_{2}=2.0 \mathrm{~Hz}, 1 \mathrm{H}\right), 7.56-7.52(\mathrm{~m}, 1 \mathrm{H}), 7.47-7.43(\mathrm{~m}, 2 \mathrm{H}), 7.33(\mathrm{~d}, J=8.8 \mathrm{~Hz}, 1 \mathrm{H}), 3.74(\mathrm{~s}, 3 \mathrm{H}) ;{ }^{13} \mathrm{C} \mathrm{NMR}$ $\left(100 \mathrm{MHz}, \mathrm{CDCl}_{3}\right): \delta=164.0,151.3,145.2,133.5,133.0,132.9,131.7,129.0,127.5,127.6\left(\mathrm{q}, J_{\mathrm{C}-\mathrm{F}}=26.2 \mathrm{~Hz}, 1\right.$ C), $123.6\left(\mathrm{q}, J_{\mathrm{C}-\mathrm{F}}=269.8 \mathrm{~Hz}, 1 \mathrm{C}\right), 126.7\left(\mathrm{q}, J_{\mathrm{C}-\mathrm{F}}=3.6 \mathrm{~Hz}, 1 \mathrm{C}\right), 124.5\left(\mathrm{q}, J_{\mathrm{C}-\mathrm{F}}=3.7 \mathrm{~Hz}, 1 \mathrm{C}\right), 114.3,30.1 ;{ }^{19} \mathrm{~F}$ NMR (376 MHz, $\mathrm{CDCl}_{3}$ ): $\delta=-62.1$; HRMS (ESI) $\mathrm{m} / \mathrm{z}$ calcd. for $\mathrm{C}_{17} \mathrm{H}_{13} \mathrm{~F}_{3} \mathrm{~N}_{3} \mathrm{O}_{2}[\mathrm{M}+\mathrm{H}]^{+}: 348.0954$, found 348.0956 .

N-(4-methyl-3-oxo-6-(trifluoromethyl)-3,4-dihydroquinoxalin-2-yl)benzamide (3oa) 
<smiles>Cn1c(=O)c(NC(=O)c2ccccc2)nc2ccc(C(F)(F)F)cc21</smiles>

${ }^{1} \mathrm{H}$ NMR $\left(400 \mathrm{MHz}, \mathrm{CDCl}_{3}\right): \delta=10.00(\mathrm{~s}, 1 \mathrm{H}), 8.05(\mathrm{~d}, J=8.4 \mathrm{~Hz}, 1 \mathrm{H}), 8.01-7.99(\mathrm{~m}, 2 \mathrm{H}), 7.65-7.61(\mathrm{~m}, 2$ $\mathrm{H}), 7.57-7.53(\mathrm{~m}, 3 \mathrm{H}), 3.85(\mathrm{~s}, 3 \mathrm{H}) ;{ }^{13} \mathrm{C} \mathrm{NMR}\left(100 \mathrm{MHz}, \mathrm{CDCl}_{3}\right): \delta=164.2,151.3,145.9,134.3\left(\mathrm{q}, J_{C-F}=1.5\right.$ $\mathrm{Hz}), 133.5,133.0,130.8,130.0,129.8\left(\mathrm{q}, J_{C-F}=32.8 \mathrm{~Hz}\right), 129.0,127.6,123.7\left(\mathrm{q}, J_{C-F}=271.3 \mathrm{~Hz}\right), 121.3\left(\mathrm{q}, J_{C-F}=\right.$ $3.6 \mathrm{~Hz}), 111.1\left(\mathrm{q}, J_{C-F}=3.7 \mathrm{~Hz}\right), 30.1 ;{ }^{19} \mathrm{~F} \mathrm{NMR}\left(376 \mathrm{MHz}, \mathrm{CDCl}_{3}\right): \delta=-62.1 ; \mathrm{HRMS}(\mathrm{ESI}) \mathrm{m} / \mathrm{z}$ calcd. for $\mathrm{C}_{17} \mathrm{H}_{13} \mathrm{~F}_{3} \mathrm{~N}_{3} \mathrm{O}_{2}[\mathrm{M}+\mathrm{H}]^{+}: 348.0954$, found 348.0947.

\section{$\mathrm{N}$-(6,7-difluoro-4-methyl-3-oxo-3,4-dihydroquinoxalin-2-yl)benzamide (3pa)}<smiles>Cn1c(=O)c(NC(=O)c2ccccc2)nc2cc(F)c(F)cc21</smiles>

${ }^{1} \mathrm{H}$ NMR (400 MHz, $\left.\mathrm{CDCl}_{3}\right): \delta=9.88(\mathrm{~s}, 1 \mathrm{H}), 7.97-7.95(\mathrm{~m}, 2 \mathrm{H}), 7.77-7.72(\mathrm{~m}, 1 \mathrm{H}), 7.62-7.58(\mathrm{~m}, 1 \mathrm{H})$, $7.54-7.50(\mathrm{~m}, 2 \mathrm{H}), 7.14-7.10(\mathrm{~m}, 1 \mathrm{H}), 3.74(\mathrm{~s}, 3 \mathrm{H}) ;{ }^{13} \mathrm{C} \mathrm{NMR}\left(100 \mathrm{MHz}, \mathrm{CDCl}_{3}\right): \delta=164.1,151.0,150.0(\mathrm{dd}$, $\left.J_{1}=250.1 \mathrm{~Hz}, J_{2}=13.9 \mathrm{~Hz}, 1 \mathrm{C}\right), 147.2\left(\mathrm{dd}, J_{1}=246.5 \mathrm{~Hz}, J_{2}=13.9 \mathrm{~Hz}, 1 \mathrm{C}\right), 144.5(\mathrm{~d}, J=2.9 \mathrm{~Hz}, 1 \mathrm{C}), 133.5$, 132.9, 129.0, 128.5, (dd, $\left.J_{1}=9.5 \mathrm{~Hz}, J_{2}=2.9 \mathrm{~Hz}, 1 \mathrm{C}\right), 127.7\left(\mathrm{dd}, J_{1}=8.7 \mathrm{~Hz}, J_{2}=2.1 \mathrm{~Hz}, 1 \mathrm{C}\right), 127.5,117.0(\mathrm{dd}$, $\left.J_{1}=19.0 \mathrm{~Hz}, J_{2}=1.5 \mathrm{~Hz}, 1 \mathrm{C}\right), 102.5(\mathrm{~d}, J=23.3 \mathrm{~Hz}, 1 \mathrm{C}), 30.4 ;{ }^{19} \mathrm{~F}$ NMR $\left(376 \mathrm{MHz}, \mathrm{CDCl}_{3}\right): \delta=-133.3\left(\mathrm{~d}, J_{F-F}\right.$ $=23.3 \mathrm{~Hz}, 1 \mathrm{~F}),-140.5\left(\mathrm{~d}, J_{F-F}=21.8 \mathrm{~Hz}, 1 \mathrm{~F}\right)$; HRMS (ESI) $\mathrm{m} / \mathrm{z}$ calcd. for $\mathrm{C}_{16} \mathrm{H}_{12} \mathrm{~F}_{2} \mathrm{~N}_{3} \mathrm{O}_{2}[\mathrm{M}+\mathrm{H}]^{+}: 316.0892$, found 316.0884 .

$N$-(4-methyl-3-oxo-3,4-dihydrobenzo[g]quinoxalin-2-yl)benzamide (3qa)<smiles>Cn1c(=O)c(NC(=O)c2ccccc2)nc2cc3ccccc3cc21</smiles>

${ }^{1} \mathrm{H}$ NMR $\left(400 \mathrm{MHz}, \mathrm{CDCl}_{3}\right): \delta=9.98(\mathrm{~s}, 1 \mathrm{H}), 8.45(\mathrm{~s}, 1 \mathrm{H}), 8.02-7.96(\mathrm{~m}, 3 \mathrm{H}), 7.90(\mathrm{~d}, J=8.4 \mathrm{~Hz}, 1 \mathrm{H}), 7.64-$ $7.60(\mathrm{~m}, 2 \mathrm{H}), 7.56-7.47$ (m, $4 \mathrm{H}), 3.86(\mathrm{~s}, 3 \mathrm{H}) ;{ }^{13} \mathrm{C} \mathrm{NMR}\left(100 \mathrm{MHz}, \mathrm{CDCl}_{3}\right): \delta=164.2,151.7,144.0,133.9$, 132.7, 131.2, 130.5, 129.9, 129.0, 128.3, 128.0, 127.6, 127.3, 127.1, 125.6, 110.4, 29.9; HRMS (ESI) m/z calcd. for $\mathrm{C}_{20} \mathrm{H}_{16} \mathrm{~N}_{3} \mathrm{O}_{2}[\mathrm{M}+\mathrm{H}]^{+}: 330.1237$, found 330.1232 .

\section{4-methyl-N-(3-oxo-4-pentyl-3,4-dihydroquinoxalin-2-yl)benzamide (3ab)}<smiles>CCCCCn1c(=O)c(NC(=O)c2ccc(C)cc2)nc2ccccc21</smiles>

${ }^{1} \mathrm{H}$ NMR (400 MHz, $\left.\mathrm{CDCl}_{3}\right): \delta=9.94(\mathrm{~s}, 1 \mathrm{H}), 7.96\left(\mathrm{dd}, J_{1}=8.0 \mathrm{~Hz}, J_{2}=1.6 \mathrm{~Hz}, 1 \mathrm{H}\right), 7.91-7.88(\mathrm{~m}, 2 \mathrm{H}), 7.50$ -7.45 (m, $1 \mathrm{H}), 7.39-7.30$ (m, $4 \mathrm{H}), 4.33-4.29$ (m, $2 \mathrm{H}), 2.43$ (s, $3 \mathrm{H}), 1.84-1.77$ (m, $2 \mathrm{H}), 1.49-1.38$ (m, 4 H), $0.93(\mathrm{t}, J=7.2 \mathrm{~Hz}, 3 \mathrm{H}) ;{ }^{13} \mathrm{C}$ NMR $\left(100 \mathrm{MHz}, \mathrm{CDCl}_{3}\right): \delta=164.1,151.2,144.4,143.4,132.5,131.0,129.9$, $129.7,129.5,128.2,127.6,124.4,113.6,43.2,29.0,27.0,22.3,21.6,13.9 ;$ HRMS (ESI) $\mathrm{m} / \mathrm{z}$ calcd. for 
$\mathrm{C}_{21} \mathrm{H}_{24} \mathrm{~N}_{3} \mathrm{O}_{2}[\mathrm{M}+\mathrm{H}]^{+}: 350.1863$, found 350.1863 .

4-(tert-butyl)-N-(3-oxo-4-pentyl-3,4-dihydroquinoxalin-2-yl)benzamide (3ac)<smiles>CCCCCn1c(=O)c(NC(=O)c2ccc(C(C)(C)C)cc2)nc2ccccc21</smiles>

${ }^{1} \mathrm{H}$ NMR $\left(400 \mathrm{MHz}, \mathrm{CDCl}_{3}\right): \delta=9.96(\mathrm{~s}, 1 \mathrm{H}), 7.98-7.94(\mathrm{~m}, 3 \mathrm{H}), 7.53(\mathrm{~d}, J=8.4 \mathrm{~Hz}, 2 \mathrm{H}), 7.48(\mathrm{t}, J=8.0 \mathrm{~Hz}$, $1 \mathrm{H}), 7.39-7.31(\mathrm{~m}, 2 \mathrm{H}), 4.32(\mathrm{t}, J=7.6 \mathrm{~Hz}, 2 \mathrm{H}), 1.83-1.79(\mathrm{~m}, 2 \mathrm{H}), 1.47-1.41(\mathrm{~m}, 4 \mathrm{H}), 1.36(\mathrm{~s}, 9 \mathrm{H}), 0.94$ $(\mathrm{t}, J=7.2 \mathrm{~Hz}, 3 \mathrm{H}) ;{ }^{13} \mathrm{C} \mathrm{NMR}\left(100 \mathrm{MHz}, \mathrm{CDCl}_{3}\right): \delta=164.1,156.4,151.2,144.4,132.5,131.0,129.9,129.7$, $128.2,127.5,125.9,124.5,113.6,43.2,35.1,31.1,29.0,27.0,22.4,13.9 ;$ HRMS (ESI) $\mathrm{m} / \mathrm{z}$ calcd. for $\mathrm{C}_{24} \mathrm{H}_{30} \mathrm{~N}_{3} \mathrm{O}_{2}[\mathrm{M}+\mathrm{H}]^{+}: 392.2333$, found 392.2329.

4-methoxy- $N$-(3-oxo-4-pentyl-3,4-dihydroquinoxalin-2-yl)benzamide (3ad)<smiles>CCCCCn1c(=O)c(NC(=O)c2ccc(OC)cc2)nc2ccccc21</smiles>

${ }^{1} \mathrm{H}$ NMR (400 MHz, $\left.\mathrm{CDCl}_{3}\right): \delta=9.89(\mathrm{~s}, 1 \mathrm{H}), 7.99-7.93(\mathrm{~m}, 3 \mathrm{H}), 7.49-7.45(\mathrm{~m}, 1 \mathrm{H}), 7.38-7.30(\mathrm{~m}, 2 \mathrm{H})$, $6.99(\mathrm{~d}, J=8.8 \mathrm{~Hz}, 2 \mathrm{H}), 4.31(\mathrm{t}, J=7.6 \mathrm{~Hz}, 2 \mathrm{H}), 3.88(\mathrm{~s}, 3 \mathrm{H}), 1.84-1.77(\mathrm{~m}, 2 \mathrm{H}), 1.47-1.38(\mathrm{~m}, 4 \mathrm{H}), 0.93(\mathrm{t}$, $J=7.2 \mathrm{~Hz}, 3 \mathrm{H}) ;{ }^{13} \mathrm{C} \mathrm{NMR}\left(100 \mathrm{MHz}, \mathrm{CDCl}_{3}\right): \delta=163.6,163.1,151.2,144.4,132.5,130.1,129.8,129.6,128.1$, 126.1, 124.4, 114.1, 113.6, 55.5, 43.2, 29.0, 27.0, 22.3, 13.9; HRMS (ESI) m/z calcd. for $\mathrm{C}_{21} \mathrm{H}_{24} \mathrm{~N}_{3} \mathrm{O}_{3}[\mathrm{M}+\mathrm{H}]^{+}$: 366.1812 , found 366.1815 .

4-fluoro- $N$-(3-oxo-4-pentyl-3,4-dihydroquinoxalin-2-yl)benzamide (3ae)<smiles>CCCCCn1c(=O)c(NC(=O)c2ccc(F)cc2)nc2ccccc21</smiles>

${ }^{1} \mathrm{H}$ NMR $\left(400 \mathrm{MHz}, \mathrm{CDCl}_{3}\right): \delta=9.89(\mathrm{~s}, 1 \mathrm{H}), 8.03-8.00(\mathrm{~m}, 2 \mathrm{H}), 7.95\left(\mathrm{dd}, J_{1}=8.0 \mathrm{~Hz}, J_{2}=1.2 \mathrm{~Hz}, 1 \mathrm{H}\right), 7.51$ - 7.47 (m, $1 \mathrm{H}), 7.40$ - 7.32 (m, $2 \mathrm{H}), 7.21-7.17$ (m, $2 \mathrm{H}), 4.32$ (t, J= 7.6 Hz, $2 \mathrm{H}), 1.84-1.77$ (m, $2 \mathrm{H}), 1.49$ $1.38(\mathrm{~m}, 4 \mathrm{H}), 0.93(\mathrm{t}, J=6.8 \mathrm{~Hz}, 3 \mathrm{H}) ;{ }^{13} \mathrm{C} \mathrm{NMR}\left(100 \mathrm{MHz}, \mathrm{CDCl}_{3}\right): \delta=165.4\left(J_{\mathrm{C}-\mathrm{F}}=252.3\right), 163.1,151.1,144.2$, $132.4,130.1,130.0,129.9,129.7,128.4,124.5,116.0\left(J_{\mathrm{C}-\mathrm{F}}=21.9\right), 113.6,43.2,29.0,27.0,22.3,13.9$; HRMS (ESI) $\mathrm{m} / \mathrm{z}$ calcd. for $\mathrm{C}_{20} \mathrm{H}_{21} \mathrm{FN}_{3} \mathrm{O}_{2}[\mathrm{M}+\mathrm{H}]^{+}: 354.1612$, found 354.1609 .

4-chloro-N-(3-oxo-4-pentyl-3,4-dihydroquinoxalin-2-yl)benzamide (3af)<smiles>CCCCCn1c(=O)c(NC(=O)c2ccc(Cl)cc2)nc2ccccc21</smiles> 
${ }^{1} \mathrm{H}$ NMR $\left(400 \mathrm{MHz}, \mathrm{CDCl}_{3}\right): \delta=9.90(\mathrm{~s}, 1 \mathrm{H}), 7.96-7.92(\mathrm{~m}, 3 \mathrm{H}), 7.51-7.47(\mathrm{~m}, 3 \mathrm{H}), 7.40-7.32(\mathrm{~m}, 2 \mathrm{H})$, $4.31(\mathrm{t}, J=7.6 \mathrm{~Hz}, 2 \mathrm{H}), 1.84-1.77(\mathrm{~m}, 2 \mathrm{H}), 1.49-1.38(\mathrm{~m}, 4 \mathrm{H}), 0.93(\mathrm{t}, J=6.8 \mathrm{~Hz}, 3 \mathrm{H}) ;{ }^{13} \mathrm{C} \mathrm{NMR}(100 \mathrm{MHz}$, $\left.\mathrm{CDCl}_{3}\right): \delta=163.2,151.1,144.1,139.1,132.3,132.2,130.0,129.7,129.2,129.0,128.5,124.6,113.7,43.2,29.0$, 27.0, 22.3, 13.9; HRMS (ESI) $\mathrm{m} / \mathrm{z}$ calcd. for $\mathrm{C}_{20} \mathrm{H}_{21} \mathrm{ClN}_{3} \mathrm{O}_{2}[\mathrm{M}+\mathrm{H}]^{+}: 370.1317$, found 370.1315.

\section{4-bromo- $N$-(3-oxo-4-pentyl-3,4-dihydroquinoxalin-2-yl)benzamide (3ag)}<smiles>CCCCCn1c(=O)c(NC(=O)c2ccc(Br)cc2)nc2ccccc21</smiles>

${ }^{1} \mathrm{H}$ NMR $\left(400 \mathrm{MHz}, \mathrm{CDCl}_{3}\right): \delta=9.90(\mathrm{~s}, 1 \mathrm{H}), 7.95(\mathrm{~d}, J=8.0 \mathrm{~Hz}, 1 \mathrm{H}), 7.87(\mathrm{~d}, J=8.8 \mathrm{~Hz}, 2 \mathrm{H}), 7.67-7.64(\mathrm{~m}$, $2 \mathrm{H}), 7.52-7.48(\mathrm{~m}, 1 \mathrm{H}), 7.40-7.32(\mathrm{~m}, 2 \mathrm{H}), 4.32(\mathrm{t}, J=7.6 \mathrm{~Hz}, 2 \mathrm{H}), 1.85-1.77$ (m, $2 \mathrm{H}), 1.47-1.39$ (m, 4 H), $0.93(\mathrm{t}, J=6.8 \mathrm{~Hz}, 3 \mathrm{H}) ;{ }^{13} \mathrm{C} \mathrm{NMR}\left(100 \mathrm{MHz}, \mathrm{CDCl}_{3}\right): \delta=163.3,151.1,144.1,132.7,132.4,132.2,130.0$, $129.8,129.1,128.5,127.7,124.6,113.7,43.3,29.0,27.0,22.3,13.9 ;$ HRMS (ESI) $\mathrm{m} / \mathrm{z}$ calcd. for $\mathrm{C}_{20} \mathrm{H}_{21} \mathrm{BrN}_{3} \mathrm{O}_{2}[\mathrm{M}+\mathrm{H}]^{+}: 414.0812$, found 414.0806 .

$\mathrm{N}$-(3-oxo-4-pentyl-3,4-dihydroquinoxalin-2-yl)-4-(trifluoromethyl)benzamide (3ah)<smiles>CCCCCn1c(=O)c(NC(=O)c2ccc(C(F)(F)F)cc2)nc2ccccc21</smiles>

${ }^{1} \mathrm{H}$ NMR (400 MHz, $\left.\mathrm{CDCl}_{3}\right): \delta=9.96(\mathrm{~s}, 1 \mathrm{H}), 8.11(\mathrm{~d}, J=8.0 \mathrm{~Hz}, 2 \mathrm{H}), 7.96\left(\mathrm{dd}, J_{1}=8.0 \mathrm{~Hz}, J_{2}=0.8 \mathrm{~Hz}, 1 \mathrm{H}\right)$, 7.79 (d, $J=8.4 \mathrm{~Hz}, 2 \mathrm{H}), 7.54-7.49$ (m, $1 \mathrm{H}), 7.42-7.34$ (m, $2 \mathrm{H}), 4.33$ (t, J= 7.6 Hz, $2 \mathrm{H}), 1.85-1.78$ (m, $2 \mathrm{H})$, $1.48-1.39$ (m, $4 \mathrm{H}), 0.94(\mathrm{t}, J=7.2 \mathrm{~Hz}, 3 \mathrm{H}) ;{ }^{13} \mathrm{C} \mathrm{NMR}\left(100 \mathrm{MHz}, \mathrm{CDCl}_{3}\right): \delta=163.0,151.1,144.0,135.7$ (q, $J$ C-F $=271.3 \mathrm{~Hz}, 1 \mathrm{C}), 134.0,132.3,129.9$ (q, $\left.J_{\mathrm{C}-\mathrm{F}}=24.1 \mathrm{~Hz}, 1 \mathrm{C}\right), 128.7,128.0,126.0$ (q, $\left.J_{\mathrm{C}-\mathrm{F}}=3.6 \mathrm{~Hz}, 1 \mathrm{C}\right)$, 124.8, 124.6, 113.7, 43.3, 29.0, 27.0, 22.3, 13.9; ${ }^{19} \mathrm{~F}$ NMR (376 MHz, $\left.\mathrm{CDCl}_{3}\right): \delta=-63.0 ; \mathrm{HRMS}(\mathrm{ESI}) \mathrm{m} / \mathrm{z}$ calcd. for $\mathrm{C}_{21} \mathrm{H}_{21} \mathrm{~F}_{3} \mathrm{~N}_{3} \mathrm{O}_{2}[\mathrm{M}+\mathrm{H}]^{+}:$404.1580, found 404.1576.

4-nitro- $N$-(3-oxo-4-pentyl-3,4-dihydroquinoxalin-2-yl)benzamide (3ai)<smiles>CCCCCn1c(=O)c(NC(=O)c2ccc([N+](=O)[O-])cc2)nc2ccccc21</smiles>

${ }^{1} \mathrm{H}$ NMR $\left(400 \mathrm{MHz}, \mathrm{CDCl}_{3}\right): \delta=9.96(\mathrm{~s}, 1 \mathrm{H}), 8.40-8.36(\mathrm{~m}, 2 \mathrm{H}), 8.18-8.14(\mathrm{~m}, 2 \mathrm{H}), 7.96\left(\mathrm{dd}, J_{1}=8.0 \mathrm{~Hz}, J_{2}\right.$ $=1.6 \mathrm{~Hz}, 1 \mathrm{H}), 7.56-7.51(\mathrm{~m}, 1 \mathrm{H}), 7.43-7.35(\mathrm{~m}, 2 \mathrm{H}), 4.34(\mathrm{t}, J=7.6 \mathrm{~Hz}, 2 \mathrm{H}), 1.86-1.80(\mathrm{~m}, 2 \mathrm{H}), 1.49-$ $1.41(\mathrm{~m}, 4 \mathrm{H}), 0.94(\mathrm{t}, J=7.2 \mathrm{~Hz}, 3 \mathrm{H}) ;{ }^{13} \mathrm{C} \mathrm{NMR}\left(100 \mathrm{MHz}, \mathrm{CDCl}_{3}\right): \delta=162.4,151.0,150.2,143.9,139.3,132.2$, $130.1,129.8,128.9,128.8,124.7,124.1,113.8,43.3,29.0,27.0,22.3,13.9$; HRMS (ESI) m/z calcd. for $\mathrm{C}_{20} \mathrm{H}_{21} \mathrm{~N}_{4} \mathrm{O}_{4}[\mathrm{M}+\mathrm{H}]^{+}:$381.1557, found 381.1555.

$\mathrm{N}$-(3-oxo-4-pentyl-3,4-dihydroquinoxalin-2-yl)-2-naphthamide (3aj) 
<smiles>CCCCCn1c(=O)c(NC(=O)c2ccc3ccccc3c2)nc2ccccc21</smiles>

${ }^{1} \mathrm{H}$ NMR $\left(400 \mathrm{MHz}, \mathrm{CDCl}_{3}\right): \delta=10.13(\mathrm{~s}, 1 \mathrm{H}), 8.52(\mathrm{~s}, 1 \mathrm{H}), 8.07\left(\mathrm{dd}, J_{1}=8.4 \mathrm{~Hz}, J_{2}=1.6 \mathrm{~Hz}, 1 \mathrm{H}\right), 8.01-7.96$ (m, $3 \mathrm{H}), 7.91(\mathrm{~d}, J=7.6 \mathrm{~Hz}, 1 \mathrm{H}), 7.64-7.57$ (m, $2 \mathrm{H}), 7.53-7.48(\mathrm{~m}, 1 \mathrm{H}), 7.42-7.34(\mathrm{~m}, 2 \mathrm{H}), 4.35(\mathrm{t}, J=7.6$ $\mathrm{Hz}, 2 \mathrm{H}), 1.87-1.80(\mathrm{~m}, 2 \mathrm{H}), 1.50-1.40(\mathrm{~m}, 4 \mathrm{H}), 0.95(\mathrm{t}, J=7.2 \mathrm{~Hz}, 3 \mathrm{H}) ;{ }^{13} \mathrm{C} \mathrm{NMR}\left(100 \mathrm{MHz}, \mathrm{CDCl}_{3}\right): \delta=$ $164.3,145.9,135.3,132.5,131.1,129.8,129.3,128.9,128.4,128.3,128.3,127.8,127.0,124.5,123.8,113.7,43.3$, 29.1, 27.0, 22.4, 13.9; HRMS (ESI) m/z calcd. for $\mathrm{C}_{24} \mathrm{H}_{24} \mathrm{~N}_{3} \mathrm{O}_{2}[\mathrm{M}+\mathrm{H}]^{+}: 386.1863$, found 386.1869.

$\mathrm{N}$-(3-oxo-4-pentyl-3,4-dihydroquinoxalin-2-yl)thiophene-2-carboxamide (3ak)<smiles>CCCCCn1c(=O)c(NC(=O)c2cccs2)nc2ccccc21</smiles>

${ }^{1} \mathrm{H}$ NMR (400 MHz, $\left.\mathrm{CDCl}_{3}\right): \delta=9.78(\mathrm{~s}, 1 \mathrm{H}), 7.94\left(\mathrm{dd}, J_{1}=8.0 \mathrm{~Hz}, J_{2}=1.2 \mathrm{~Hz}, 1 \mathrm{H}\right), 7.77(\mathrm{~d}, J=8.0 \mathrm{~Hz}, 1 \mathrm{H})$, $7.63\left(\mathrm{dd}, J_{1}=5.2 \mathrm{~Hz}, J_{2}=1.2 \mathrm{~Hz}, 1 \mathrm{H}\right), 7.50-7.46(\mathrm{~m}, 1 \mathrm{H}), 7.39-7.31(\mathrm{~m}, 2 \mathrm{H}), 7.17-7.15(\mathrm{~m}, 1 \mathrm{H}), 4.32(\mathrm{t}, J$ $=7.6 \mathrm{~Hz}, 2 \mathrm{H}), 1.85-1.77(\mathrm{~m}, 2 \mathrm{H}), 1.48-1.39(\mathrm{~m}, 4 \mathrm{H}), 0.93(\mathrm{t}, J=7.2 \mathrm{~Hz}, 3 \mathrm{H}) ;{ }^{13} \mathrm{C} \mathrm{NMR}\left(100 \mathrm{MHz}, \mathrm{CDCl}_{3}\right)$ : $\delta=158.5,151.0,144.0,138.8,132.5,132.4,129.9,129.7,129.5,128.3,128.0,124.5,113.6,43.2,29.0,27.0,22.4$, 13.9; HRMS (ESI) m/z calcd. for $\mathrm{C}_{18} \mathrm{H}_{20} \mathrm{~N}_{3} \mathrm{O}_{2} \mathrm{~S}[\mathrm{M}+\mathrm{H}]^{+}: 342.1271$, found 342.1267 .

$\mathrm{N}$-(3-oxo-4-pentyl-3,4-dihydroquinoxalin-2-yl)butyramide (3al)<smiles>CCCCCn1c(=O)c(NC(=O)CCC)nc2ccccc21</smiles>

${ }^{1} \mathrm{H}$ NMR $\left(400 \mathrm{MHz}, \mathrm{CDCl}_{3}\right): \delta=9.12(\mathrm{~s}, 1 \mathrm{H}), 7.84\left(\mathrm{dd}, J_{1}=8.0 \mathrm{~Hz}, J_{2}=1.2 \mathrm{~Hz}, 1 \mathrm{H}\right), 7.47-7.43(\mathrm{~m}, 1 \mathrm{H}), 7.36$ $-7.28(\mathrm{~m}, 2 \mathrm{H}), 4.28(\mathrm{t}, J=7.2 \mathrm{~Hz}, 2 \mathrm{H}), 1.81-1.74(\mathrm{~m}, 3 \mathrm{H}), 1.45-1.37$ (m, $4 \mathrm{H}), 1.30-1.28(\mathrm{~m}, 6 \mathrm{H}), 0.92(\mathrm{t}$, $J=7.2 \mathrm{~Hz}, 3 \mathrm{H}) ;{ }^{13} \mathrm{C} \mathrm{NMR}\left(100 \mathrm{MHz}, \mathrm{CDCl}_{3}\right): \delta=175.7,150.8,144.0,132.3,129.8,129.3,128.0,124.3,113.6$, 43.1, 36.4, 29.0, 26.9, 22.3, 19.1, 13.9; HRMS (ESI) m/z calcd. for $\mathrm{C}_{17} \mathrm{H}_{24} \mathrm{~N}_{3} \mathrm{O}_{2}[\mathrm{M}+\mathrm{H}]^{+}: 302.1863$, found 302.1861 .

$\mathrm{N}$-(3-0xo-4-pentyl-3,4-dihydroquinoxalin-2-yl)isobutyramide (3am)<smiles>CCCCCn1c(=O)c(NC(=O)C(C)C)nc2ccccc21</smiles>

${ }^{1} \mathrm{H}$ NMR (400 MHz, CDCl$): \delta=9.04(\mathrm{~s}, 1 \mathrm{H}), 7.80\left(\mathrm{dd}, J_{1}=8.0 \mathrm{~Hz}, J_{2}=1.2 \mathrm{~Hz}, 1 \mathrm{H}\right), 7.47-7.43(\mathrm{~m}, 1 \mathrm{H}), 7.35$ $-7.33(\mathrm{~m}, 1 \mathrm{H}), 7.30-7.27(\mathrm{~m}, 1 \mathrm{H}), 4.27(\mathrm{t}, J=7.6 \mathrm{~Hz}, 2 \mathrm{H}), 2.79(\mathrm{t}, J=7.2 \mathrm{~Hz}, 2 \mathrm{H}), 1.84-1.73(\mathrm{~m}, 4 \mathrm{H}), 1.45$ $-1.38(\mathrm{~m}, 4 \mathrm{H}), 1.04(\mathrm{t}, J=7.2 \mathrm{~Hz}, 3 \mathrm{H}), 0.92(\mathrm{t}, J=7.2 \mathrm{~Hz}, 3 \mathrm{H}) ;{ }^{13} \mathrm{C} \mathrm{NMR}\left(100 \mathrm{MHz}, \mathrm{CDCl}_{3}\right): \delta=172.6,150.7$, 143.9, 132.1, 129.9, 129.0, 127.9, 124.3, 113.6, 43.1, 39.8, 28.9, 26.9, 22.3, 18.2, 13.9, 13.8; HRMS (ESI) m/z calcd. for $\mathrm{C}_{17} \mathrm{H}_{24} \mathrm{~N}_{3} \mathrm{O}_{2}[\mathrm{M}+\mathrm{H}]^{+}: 302.1863$, found 302.1860 .

$N$-(3-oxo-4-pentyl-3,4-dihydroquinoxalin-2-yl)pivalamide (3an) 
<smiles>CCCCCn1c(=O)c(NC(=O)C(C)(C)C)nc2ccccc21</smiles>

${ }^{1} \mathrm{H}$ NMR $\left(400 \mathrm{MHz}, \mathrm{CDCl}_{3}\right): \delta=9.50(\mathrm{~s}, 1 \mathrm{H}), 7.90\left(\mathrm{dd}, J_{1}=8.0 \mathrm{~Hz}, J_{2}=1.6 \mathrm{~Hz}, 1 \mathrm{H}\right), 7.47-7.43(\mathrm{~m}, 1 \mathrm{H}), 7.36$ -7.27 (m, $2 \mathrm{H}), 4.27$ (t, $J=7.6 \mathrm{~Hz}, 2 \mathrm{H}), 1.82-1.74$ (m, $2 \mathrm{H}), 1.45-1.39$ (m, $4 \mathrm{H}), 1.36$ (s, $9 \mathrm{H}), 0.92(\mathrm{t}, J=7.2$ $\mathrm{Hz}, 3 \mathrm{H}) ;{ }^{13} \mathrm{C} \mathrm{NMR}\left(100 \mathrm{MHz}, \mathrm{CDCl}_{3}\right): \delta=176.0,151.1,144.2,132.4,129.8,129.6,128.1,124.4,113.5,43.2$, 40.7, 29.0, 27.3, 26.9, 22.3, 13.9; HRMS (ESI) m/z calcd. for $\mathrm{C}_{18} \mathrm{H}_{26} \mathrm{~N}_{3} \mathrm{O}_{2}[\mathrm{M}+\mathrm{H}]^{+}: 316.2020$, found 316.2019.

$\mathrm{N}$-(3-oxo-4-pentyl-3,4-dihydroquinoxalin-2-yl)cyclopropanecarboxamide (3ao)<smiles>CCCCCn1c(=O)c(NC(=O)C2CC2)nc2ccccc21</smiles>

${ }^{1} \mathrm{H}$ NMR $\left(400 \mathrm{MHz}, \mathrm{CDCl}_{3}\right): \delta=9.24(\mathrm{~s}, 1 \mathrm{H}), 7.80\left(\mathrm{dd}, J_{1}=8.0 \mathrm{~Hz}, J_{2}=1.2 \mathrm{~Hz}, 1 \mathrm{H}\right), 7.46-7.42(\mathrm{~m}, 1 \mathrm{H}), 7.34$ -7.27 (m, $2 \mathrm{H}), 4.30-4.26$ (m, $2 \mathrm{H}), 2.38$ (brs, $1 \mathrm{H}), 1.81-1.74$ (m, $2 \mathrm{H}), 1.44-1.37$ (m, $4 \mathrm{H}), 1.23-1.19$ (m, 2 $\mathrm{H}), 1.00-0.99$ (m, $5 \mathrm{H}) ;{ }^{13} \mathrm{C} \mathrm{NMR}\left(100 \mathrm{MHz}, \mathrm{CDCl}_{3}\right): \delta=172.9,150.8,144.0,132.1,129.9,129.1,127.9,124.3$, 113.6, 43.1, 28.9, 26.9, 22.3, 15.5, 13.9, 9.74; HRMS (ESI) $\mathrm{m} / \mathrm{z}$ calcd. for $\mathrm{C}_{17} \mathrm{H}_{22} \mathrm{~N}_{3} \mathrm{O}_{2}[\mathrm{M}+\mathrm{H}]^{+}: 300.1707$, found 300.1702 .

N-(3-oxo-4-pentyl-3,4-dihydroquinoxalin-2-yl)cyclohexanecarboxamide (3ap)<smiles>CCCCCn1c(=O)c(NC(=O)C2CCCCC2)nc2ccccc21</smiles>

${ }^{1} \mathrm{H}$ NMR (400 MHz, CDCl $)$ ): $\delta=9.11(\mathrm{~s}, 1 \mathrm{H}), 7.84\left(\mathrm{dd}, J_{1}=8.0 \mathrm{~Hz}, J_{2}=0.8 \mathrm{~Hz}, 1 \mathrm{H}\right), 7.48-7.43(\mathrm{~m}, 1 \mathrm{H}), 7.36$ -7.28 (m, $2 \mathrm{H}), 4.30-4.26$ (m, $2 \mathrm{H}), 2.82$ (brs, $1 \mathrm{H}), 2.05-2.02(\mathrm{~m}, 2 \mathrm{H}), 1.87-1.74(\mathrm{~m}, 5 \mathrm{H}), 1.63-1.55(\mathrm{~m}, 2$ H), $1.45-1.31(\mathrm{~m}, 7 \mathrm{H}), 0.93(\mathrm{t}, J=7.2 \mathrm{~Hz}, 3 \mathrm{H}) ;{ }^{13} \mathrm{C} \mathrm{NMR}\left(100 \mathrm{MHz}, \mathrm{CDCl}_{3}\right): \delta=150.9,144.0,132.3,131.0$, 129.9, 129.3, 128.0, 124.3, 113.6, 43.1, 29.2, 29.0, 25.7, 25.6, 22.4, 13.9; HRMS (ESI) m/z calcd. for $\mathrm{C}_{20} \mathrm{H}_{28} \mathrm{~N}_{3} \mathrm{O}_{2}$ $[\mathrm{M}+\mathrm{H}]^{+}: 342.2176$, found 342.2173 .

\section{References}

1. Yuan, J.; Zhu, J.; Fu, J.; Yang, L.; Xiao, Y.; Mao, P.; Du, X.; Qu, L., Highly efficient copper-catalyzed direct $\mathrm{C}-\mathrm{H}$ amidation of quinoxalin-2(1H)-ones with amidates under microwave irradiation. Org.Chem.Front. 2019, 6 (7), 925-935. 
${ }^{1} \mathrm{H}$ and ${ }^{13} \mathrm{C}$ NMR spectra of products

$N$-(3-oxo-4-pentyl-3,4-dihydroquinoxalin-2-yl)benzamide (3aa)

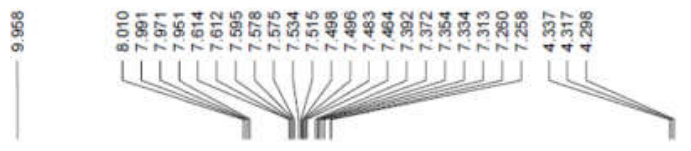

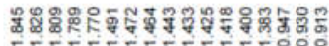
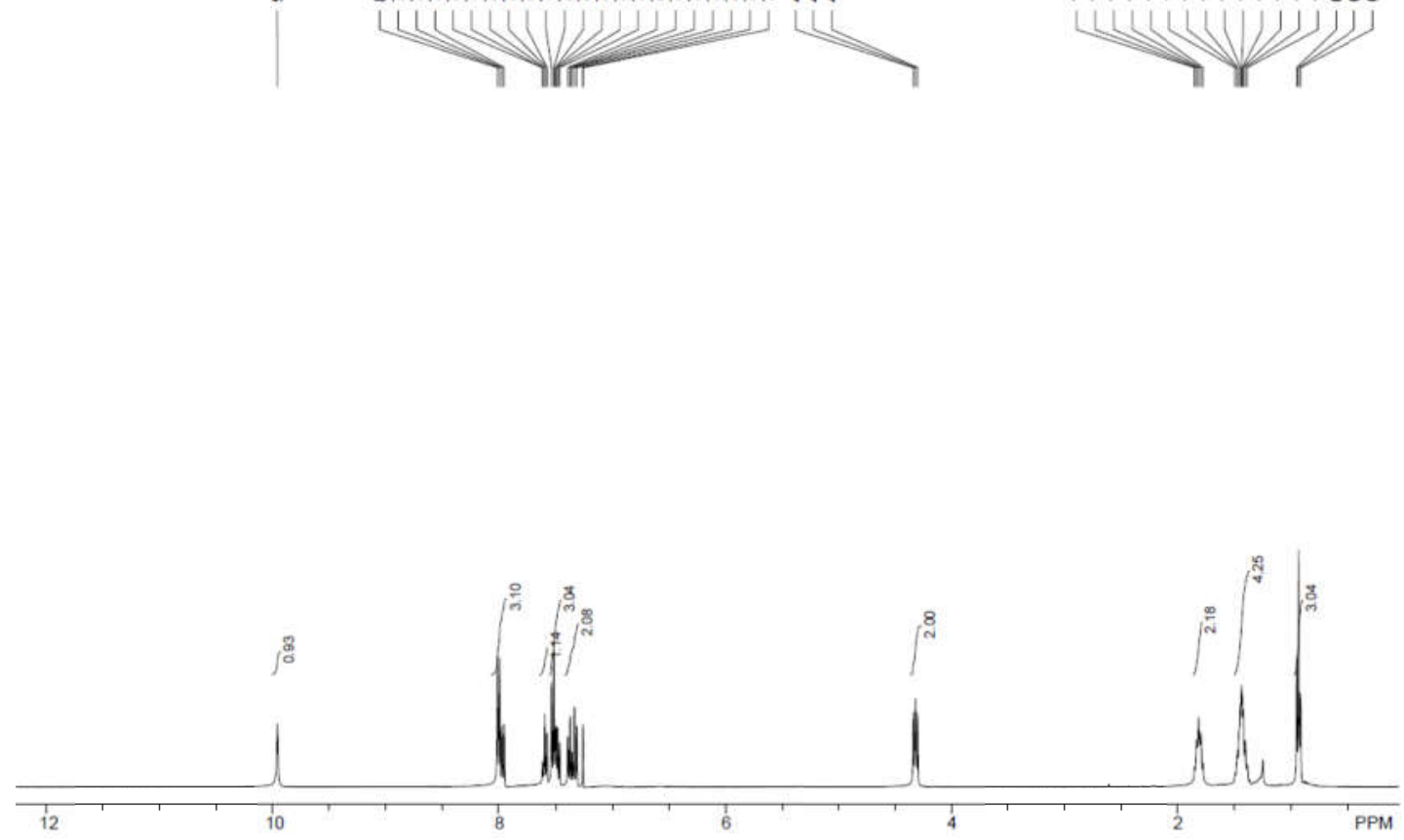

Figure S1 $\quad{ }^{1} \mathrm{H}$ spectra of $\mathbf{3 a a}$

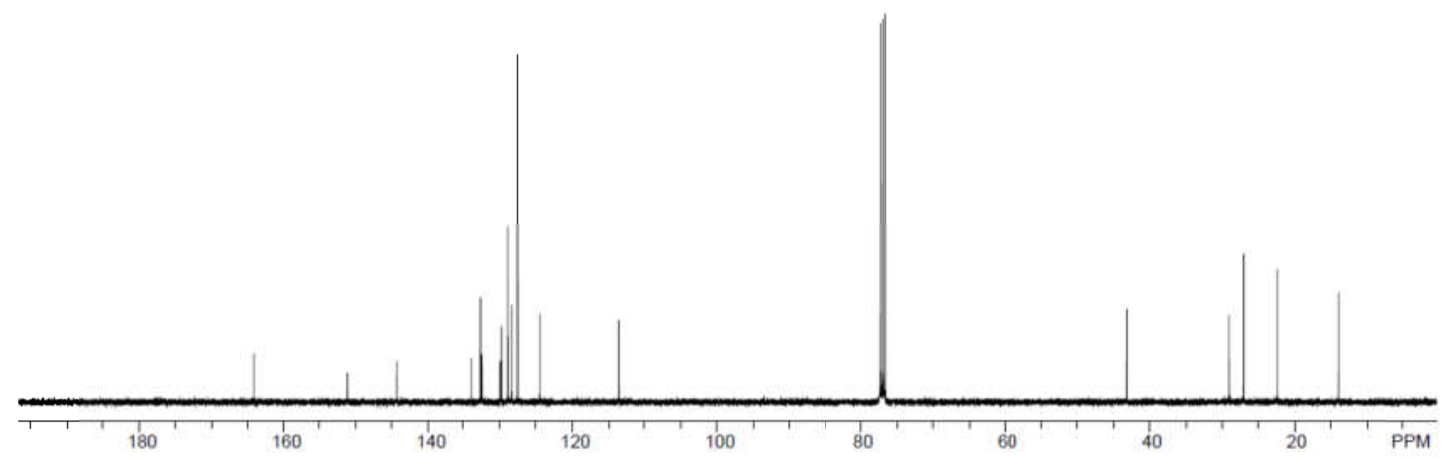

Figure S2 $\quad{ }^{13} \mathrm{C}$ spectra of $\mathbf{3 a a}$ 
$N$-(4-methyl-3-oxo-3,4-dihydroquinoxalin-2-yl)benzamide (3ba)
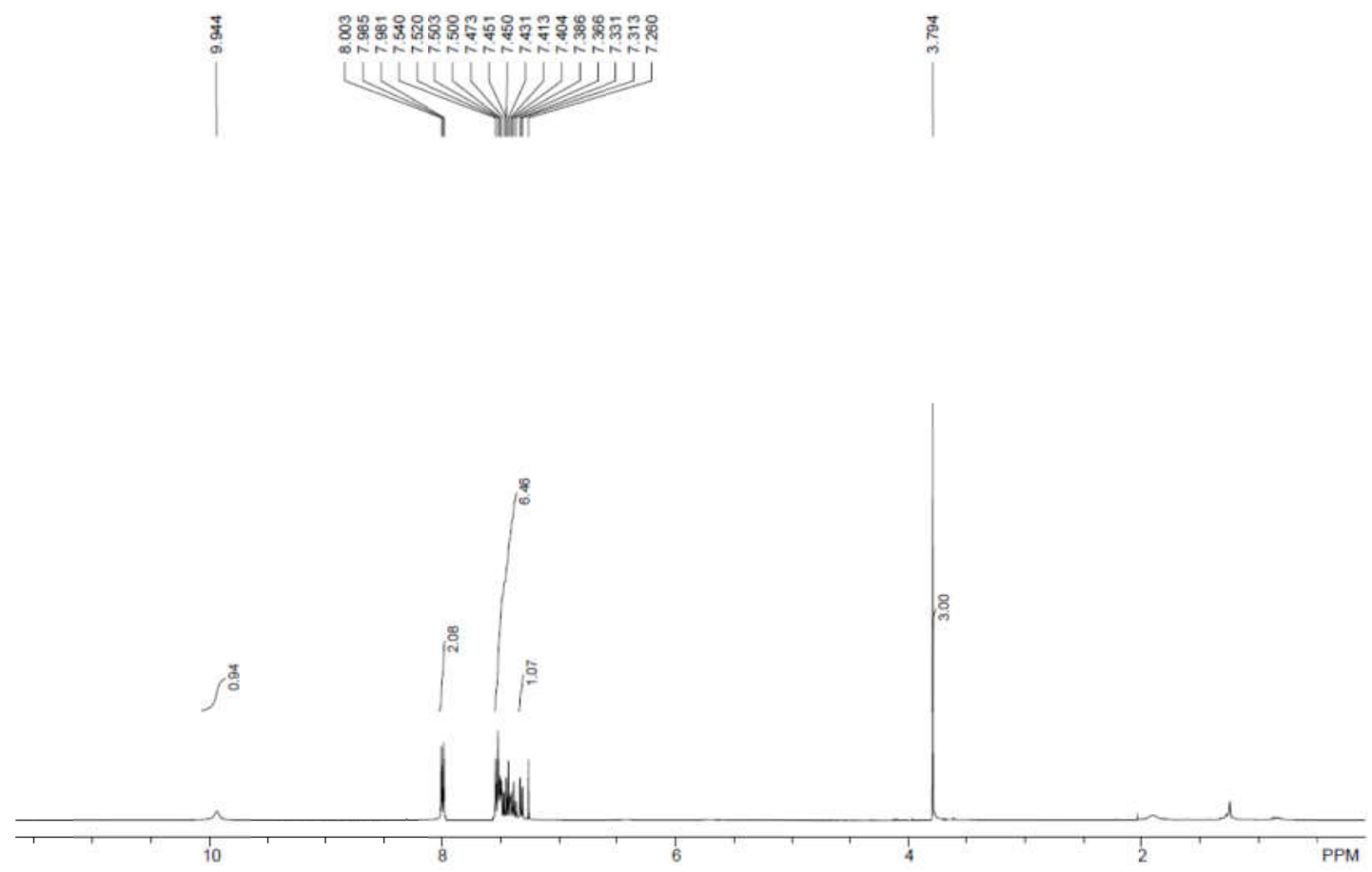

Figure S3 $\quad{ }^{1} \mathrm{H}$ spectra of $\mathbf{3 b a}$

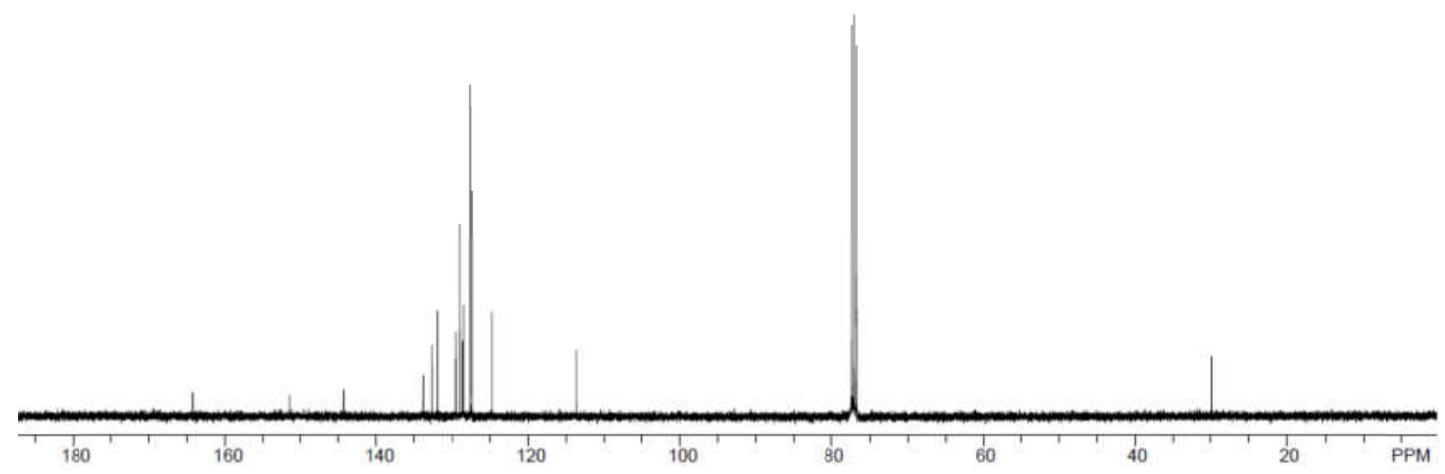

Figure $\mathbf{S 4} \quad{ }^{13} \mathrm{C}$ spectra of $\mathbf{3 b a}$ 
$N$-(4-ethyl-3-oxo-3,4-dihydroquinoxalin-2-yl)benzamide (3ca)
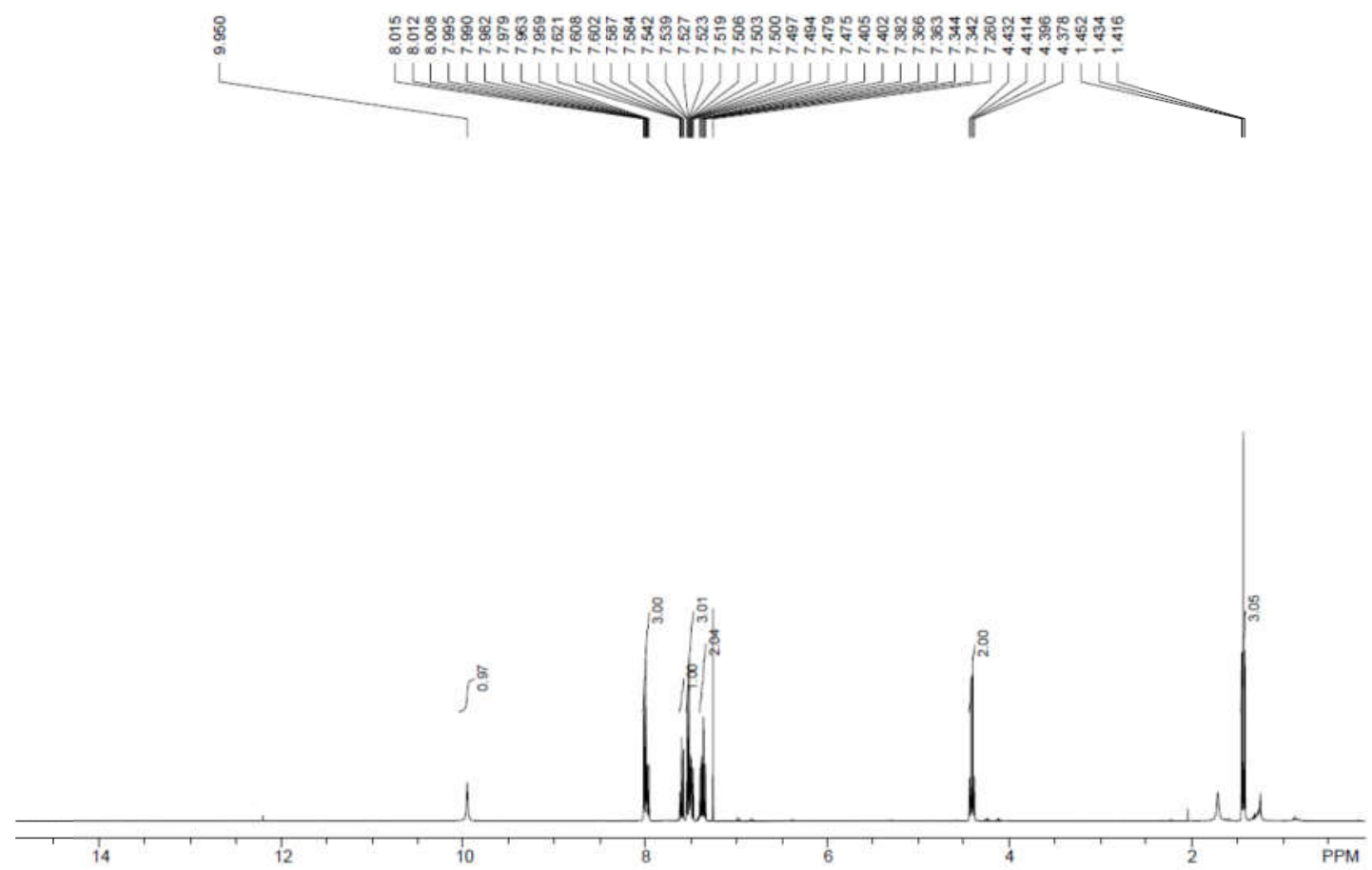

Figure S5 $\quad{ }^{1} \mathrm{H}$ spectra of $\mathbf{3 c a}$

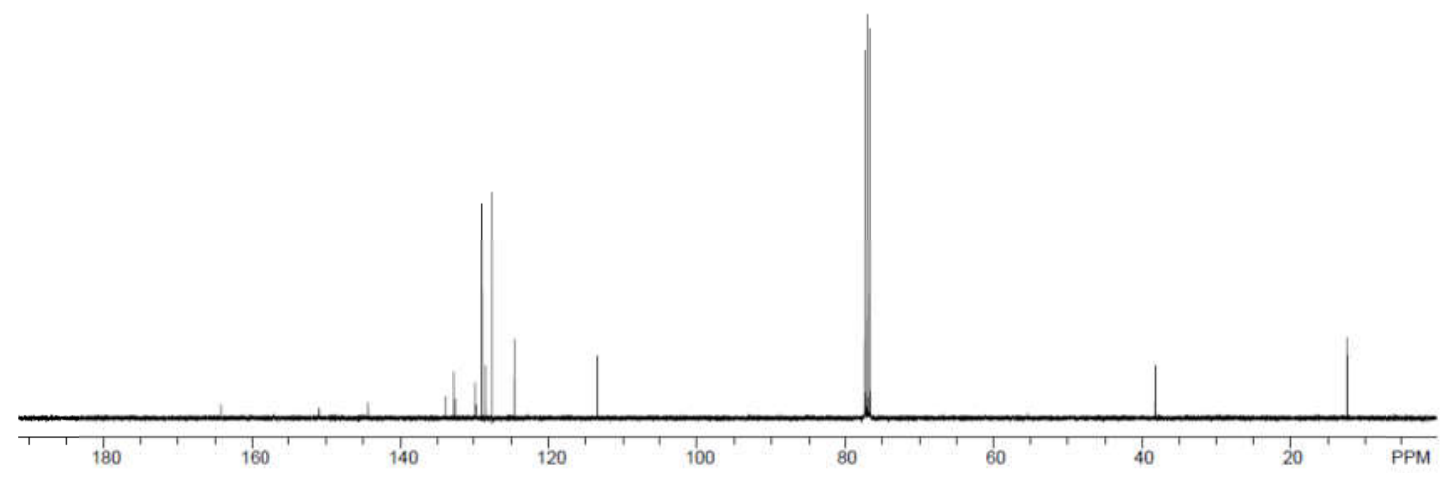

Figure S6 $\quad{ }^{13} \mathrm{C}$ spectra of $\mathbf{3 c a}$ 
$N$-(4-benzyl-3-oxo-3,4-dihydroquinoxalin-2-yl)benzamide (3da)
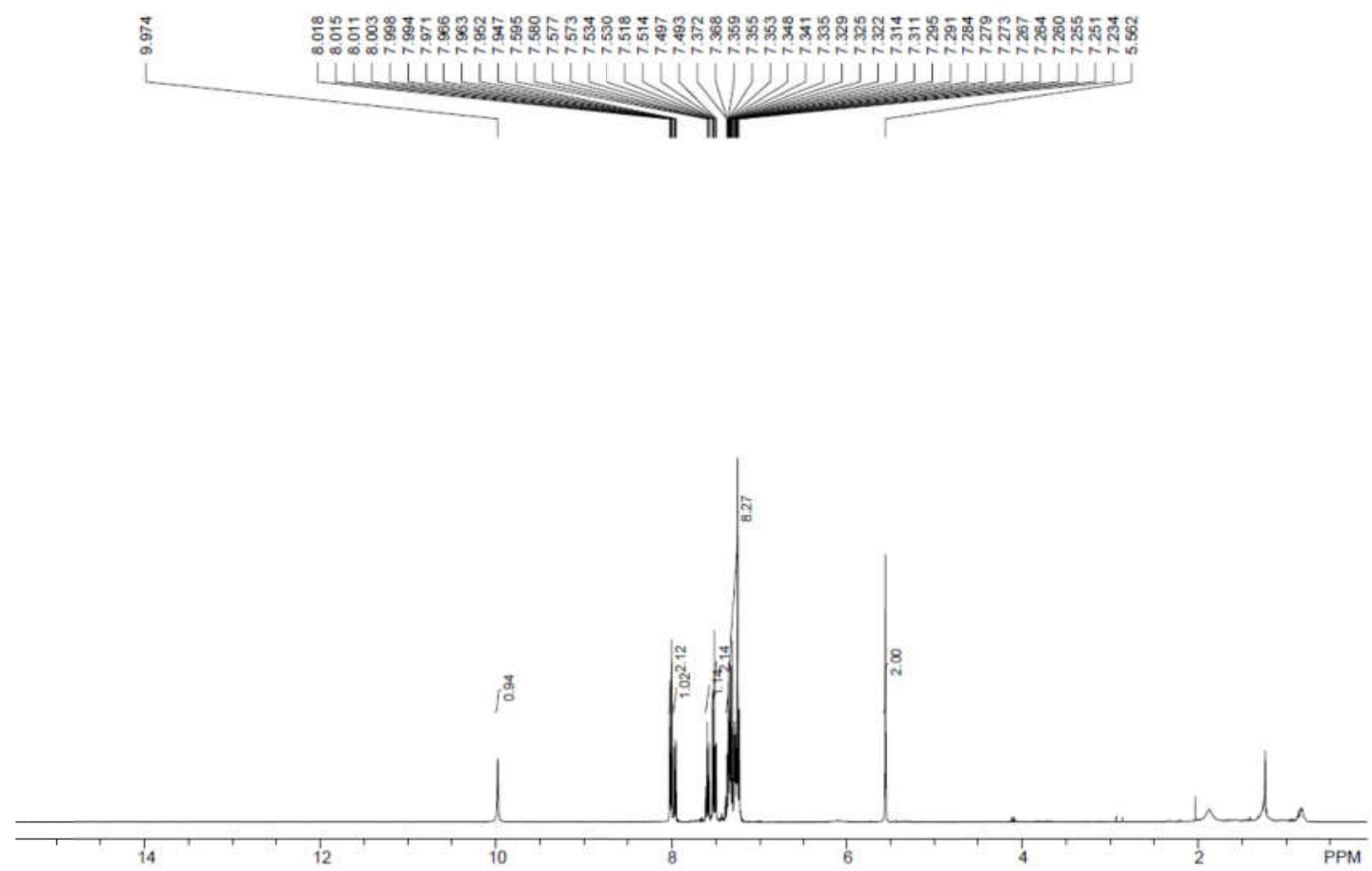

Figure S7 $\quad{ }^{1} \mathrm{H}$ spectra of $\mathbf{3 d a}$

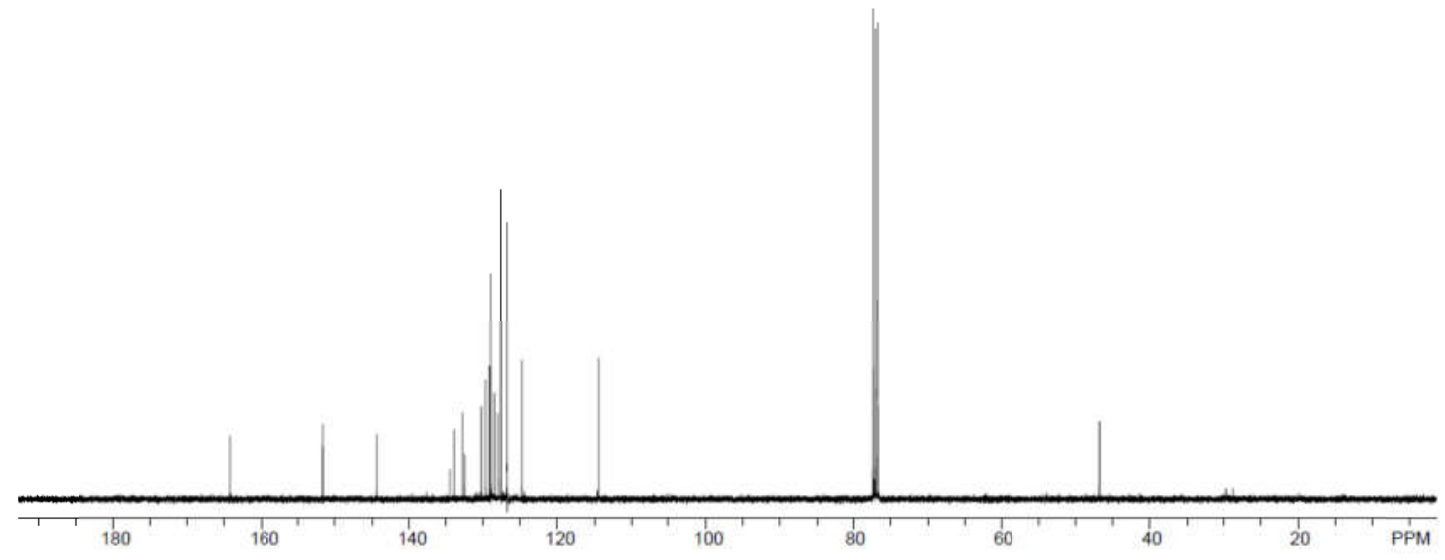

Figure $\mathbf{S 8} \quad{ }^{13} \mathrm{C}$ spectra of $\mathbf{3 d a}$ 
$N$-(4-(4-methoxybenzyl)-3-oxo-3,4-dihydroquinoxalin-2-yl)benzamide (3ea)
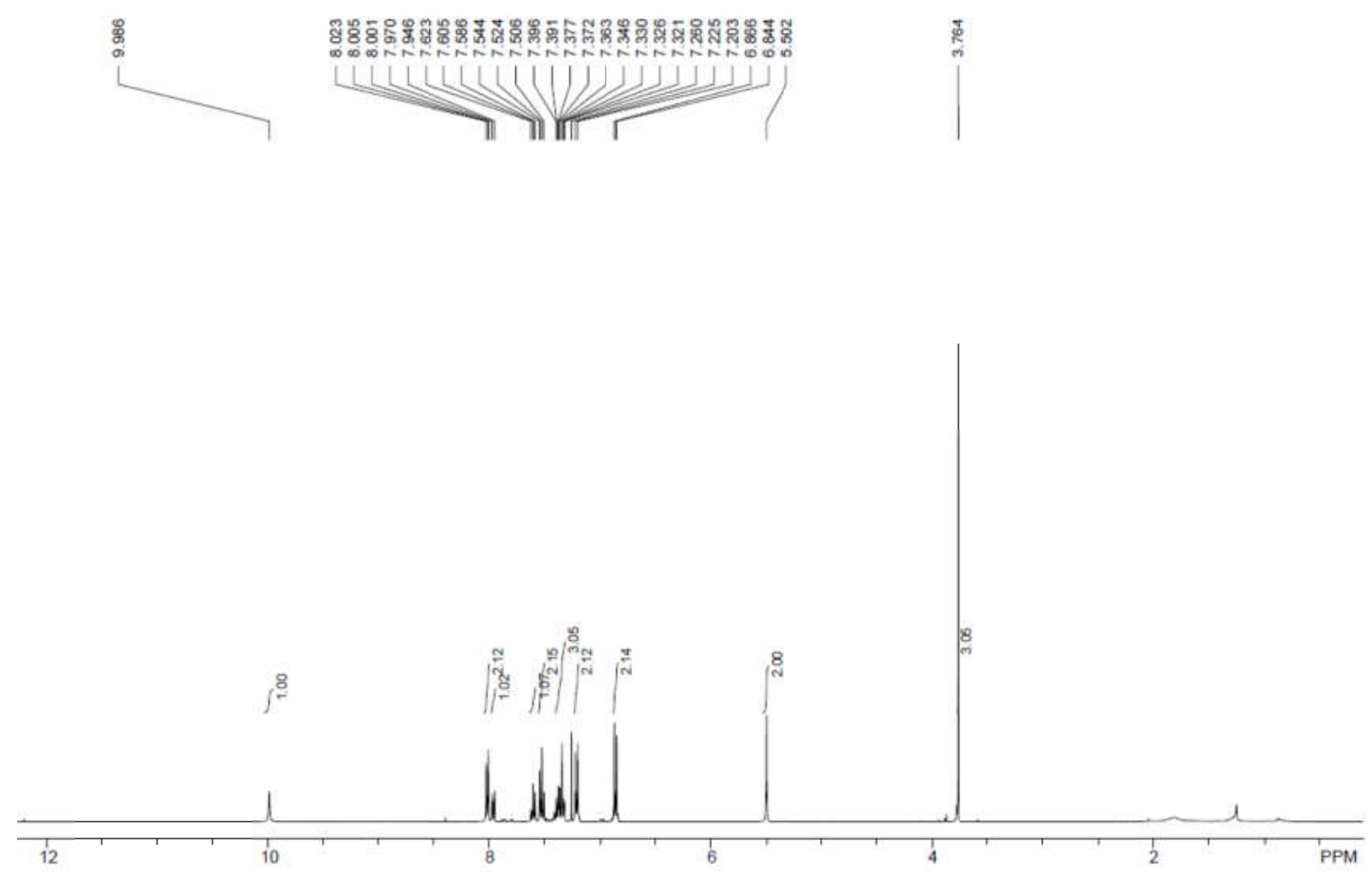

Figure S9 ${ }^{1} \mathrm{H}$ spectra of $\mathbf{3 e a}$

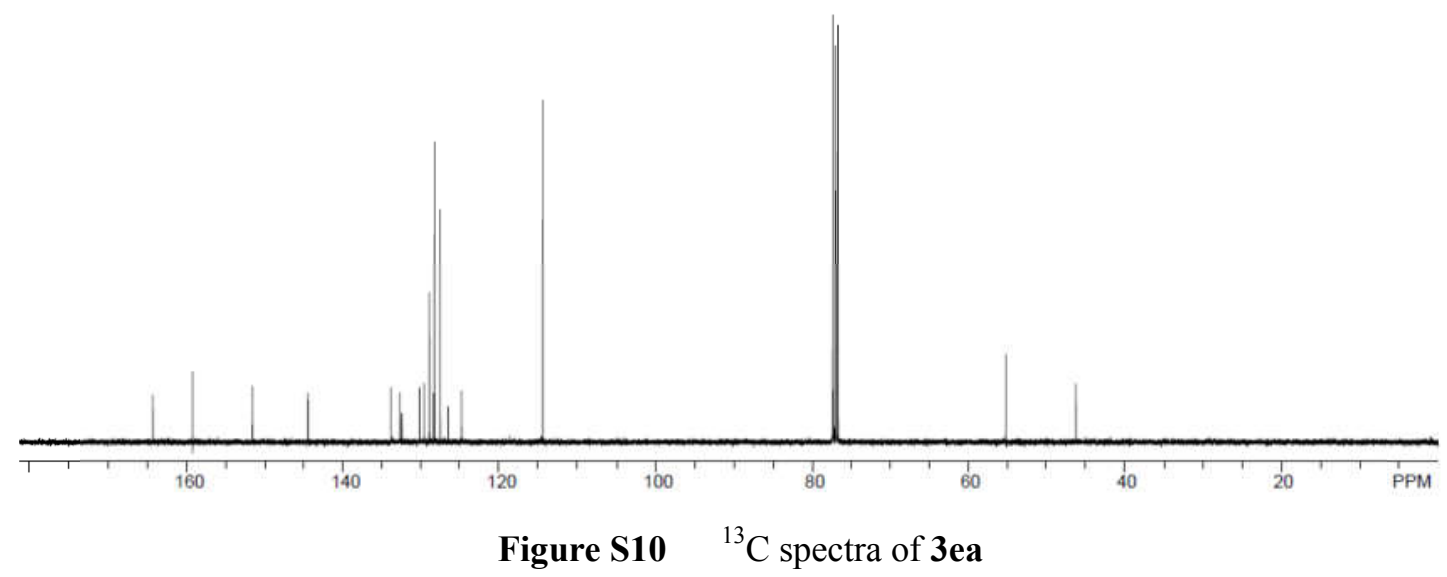


ethyl 2-(3-benzamido-2-oxoquinoxalin-1(2H)-yl)acetate (3fa)
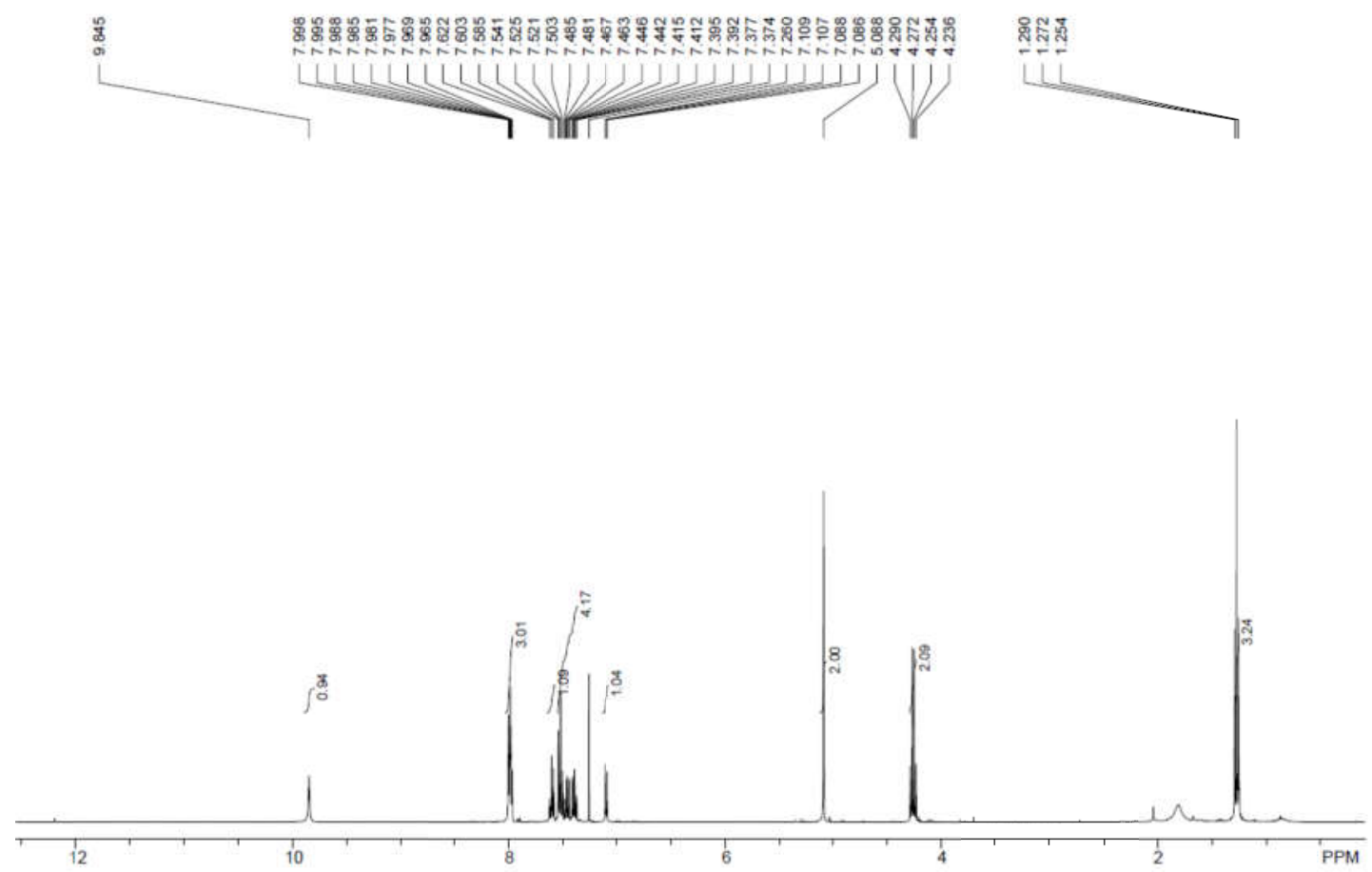

Figure S11 ${ }^{1} \mathrm{H}$ spectra of $\mathbf{3}$ fa

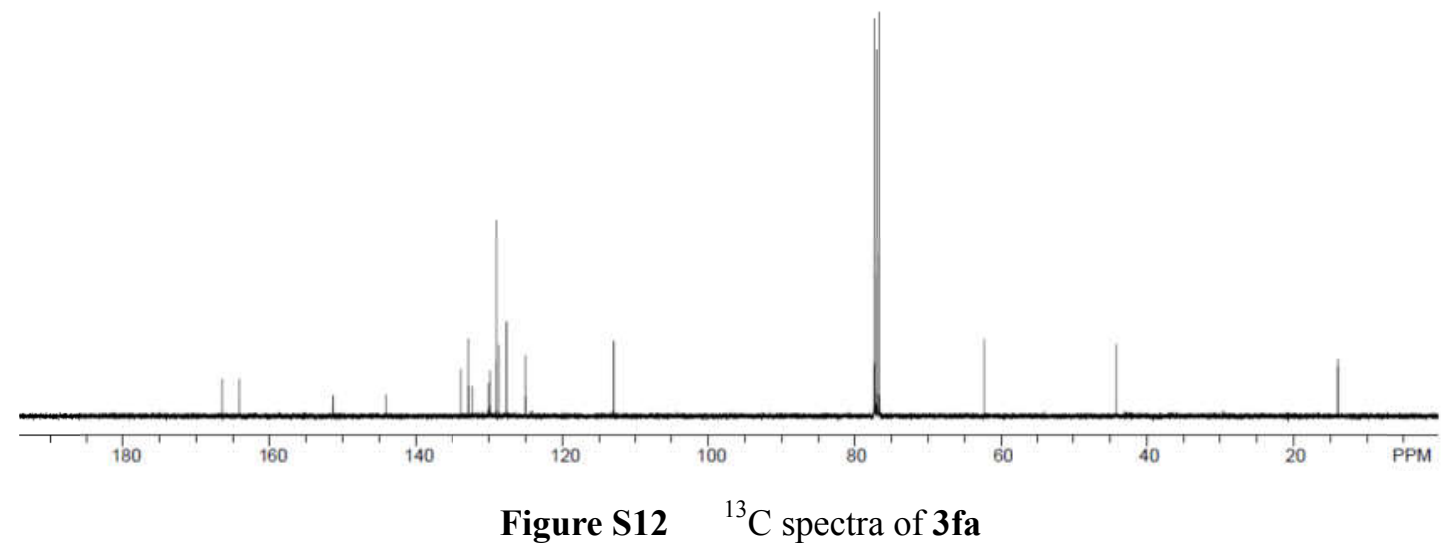


tert-butyl 2-(3-benzamido-2-oxoquinoxalin-1(2H)-yl)acetate (3ga)

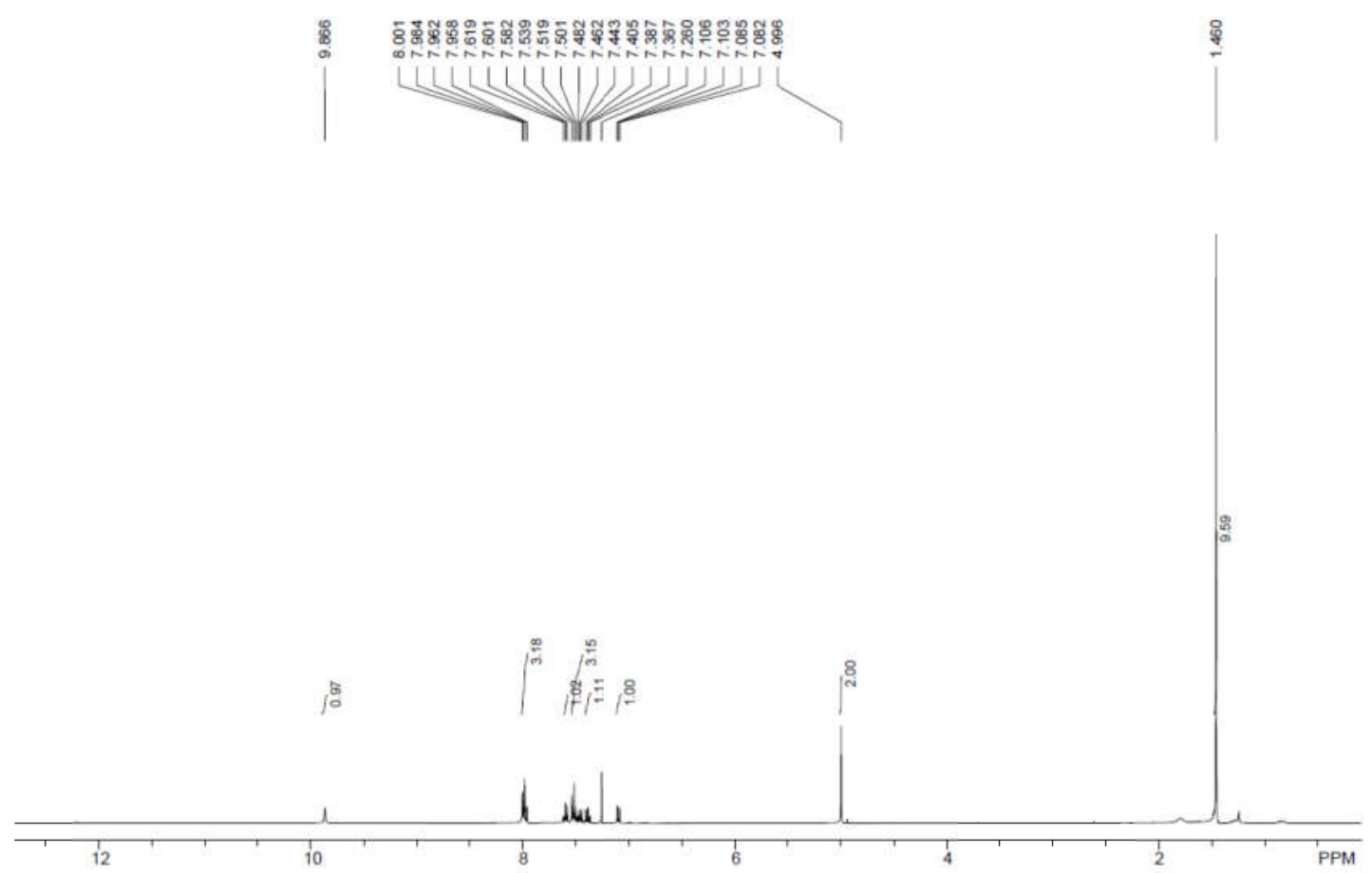

Figure S13 ${ }^{1} \mathrm{H}$ spectra of $3 g a$

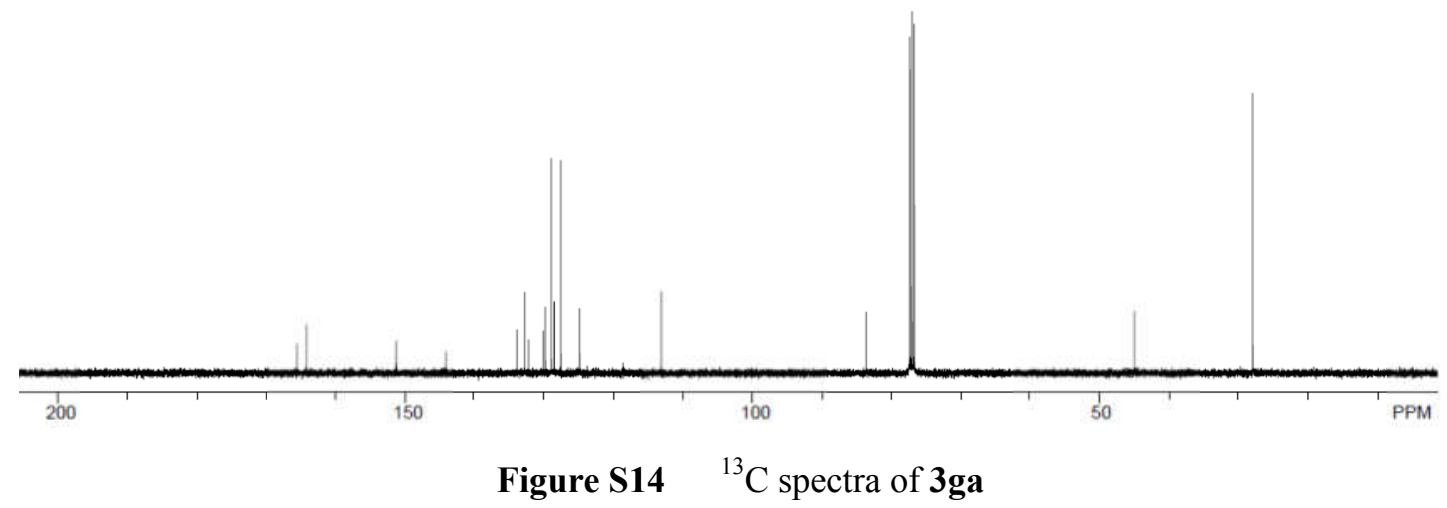


$N$-(4-allyl-3-oxo-3,4-dihydroquinoxalin-2-yl)benzamide (3ha)

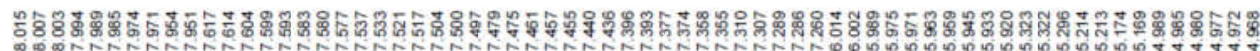
D.

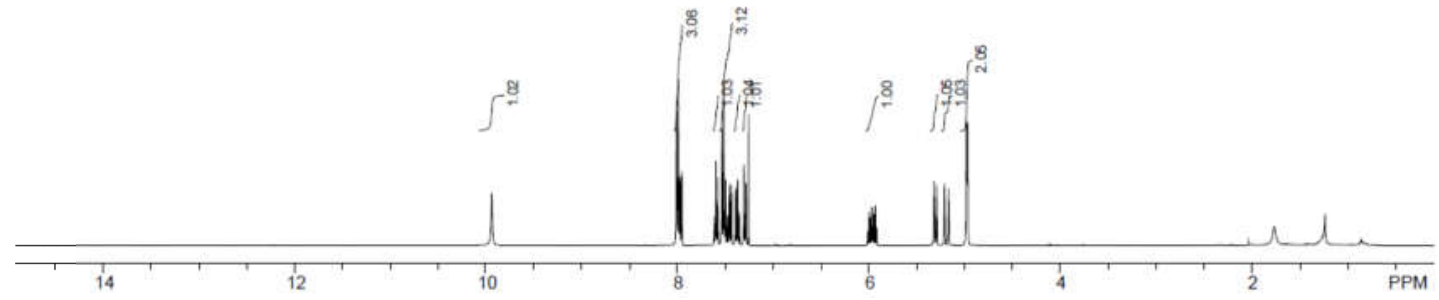

Figure S15 $\quad{ }^{1} \mathrm{H}$ spectra of $\mathbf{3 h a}$

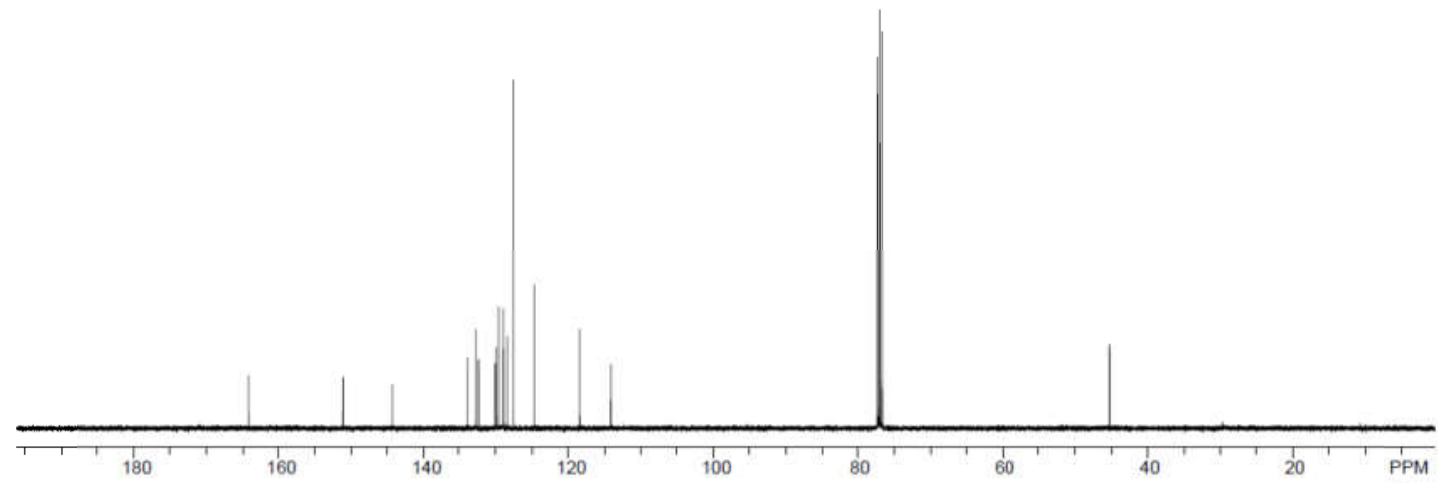

Figure $\mathbf{S 1 6} \quad{ }^{13} \mathrm{C}$ spectra of $\mathbf{3 h a}$ 
$N$-(3-0xo-4-(prop-2-yn-1-yl)-3,4-dihydroquinoxalin-2-yl)benzamide (3ia)
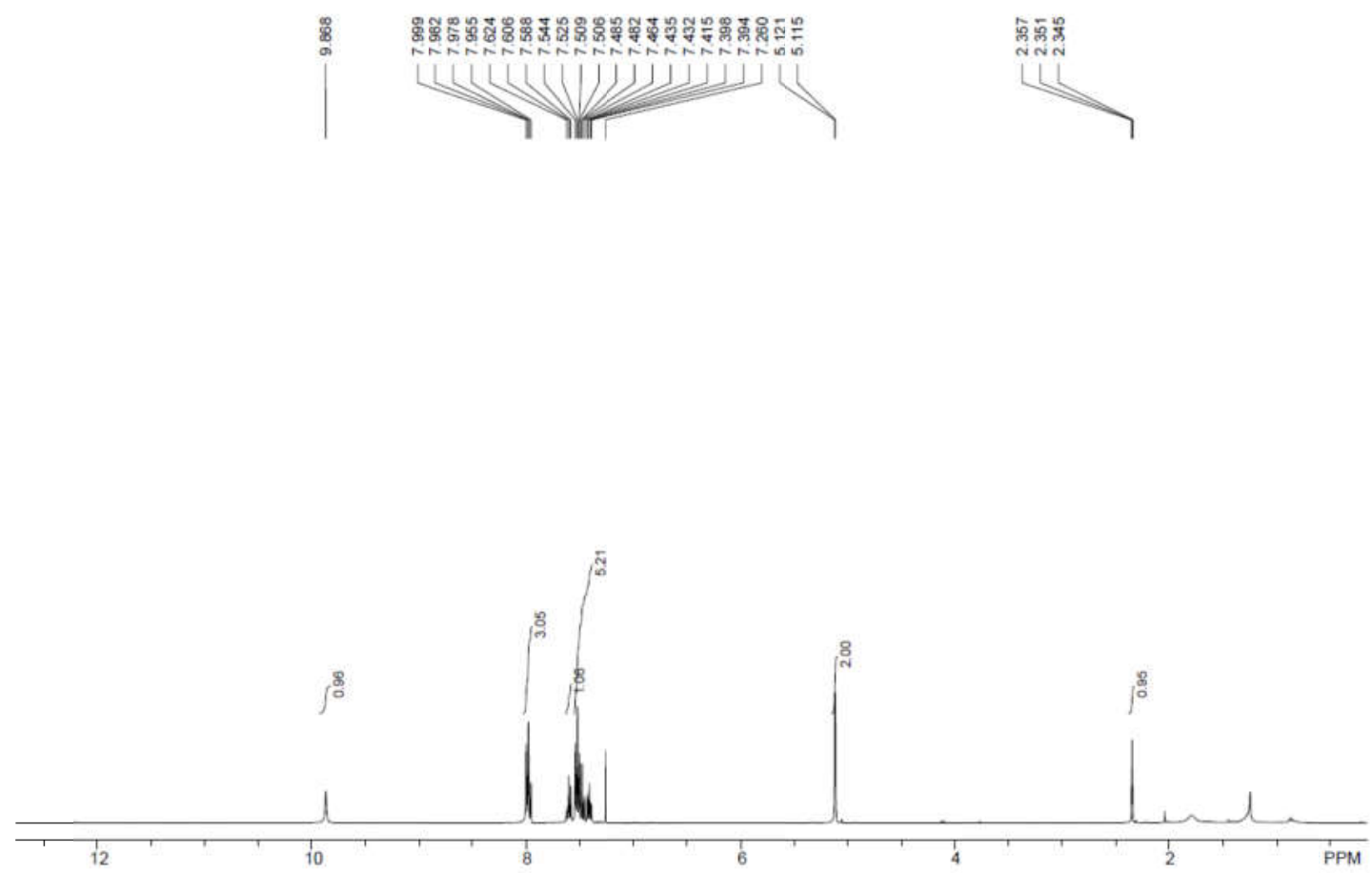

Figure $\mathbf{S 1 7}{ }^{1} \mathrm{H}$ spectra of $\mathbf{3 i a}$

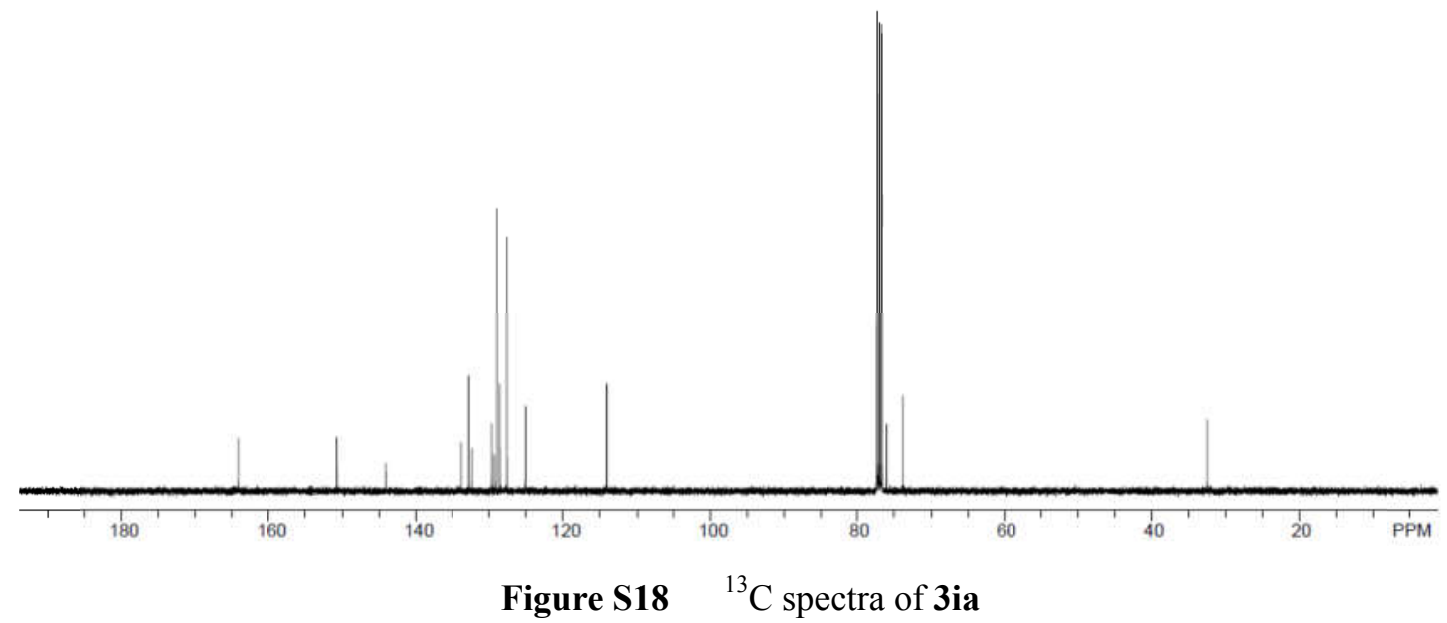


$N$-(3-oxo-4-phenyl-3,4-dihydroquinoxalin-2-yl)benzamide (3ja)
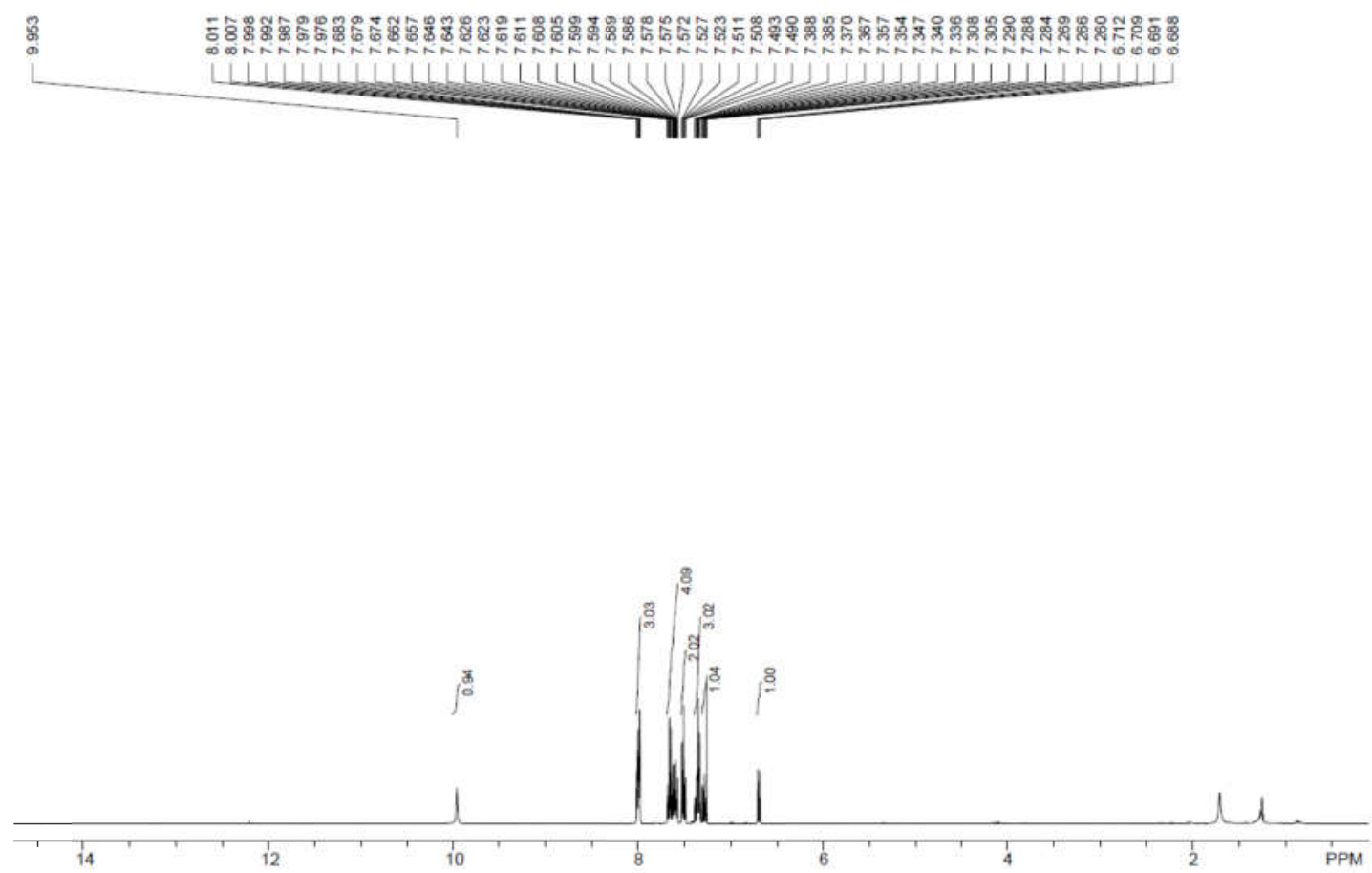

Figure S19 $\quad{ }^{1} \mathrm{H}$ spectra of $\mathbf{3 j a}$

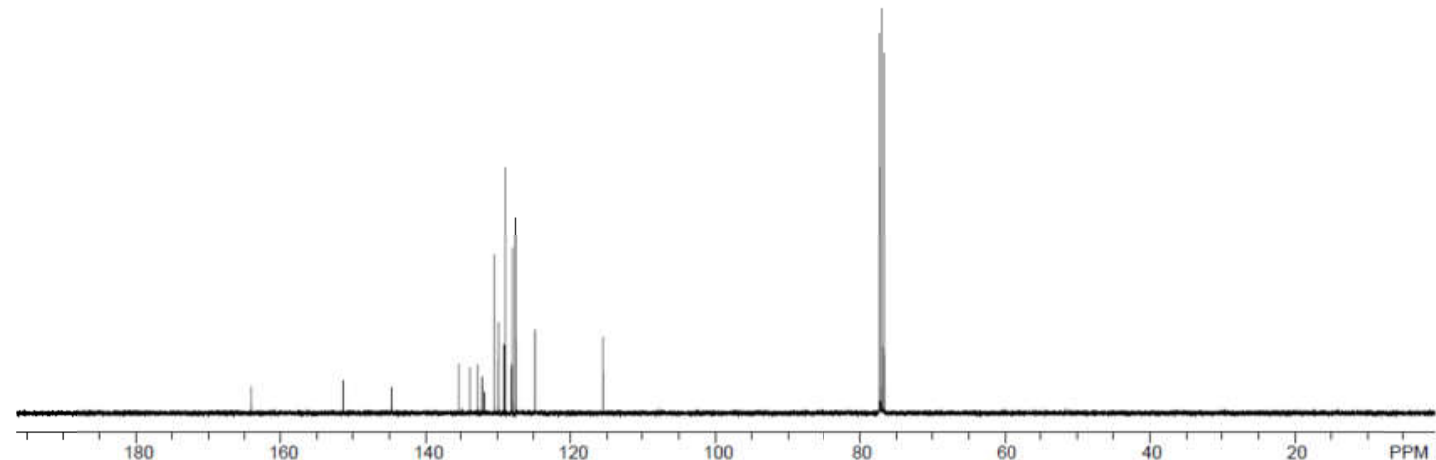

Figure S20 $\quad{ }^{13} \mathrm{C}$ spectra of $\mathbf{3 j a}$ 
$N$-(7-fluoro-4-methyl-3-oxo-3,4-dihydroquinoxalin-2-yl)benzamide (3ka)
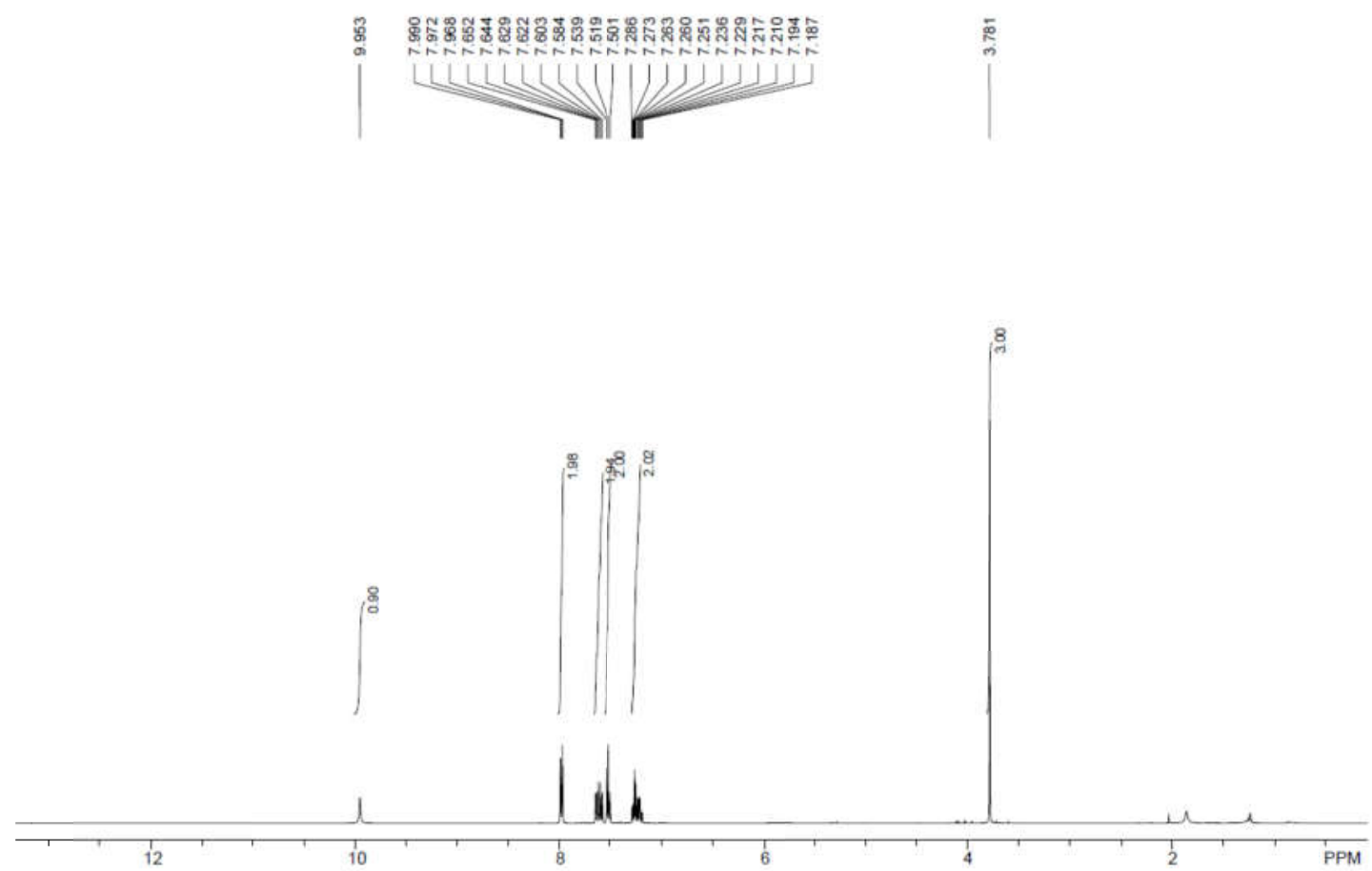

Figure S21 ${ }^{1} \mathrm{H}$ spectra of $\mathbf{3 k a}$

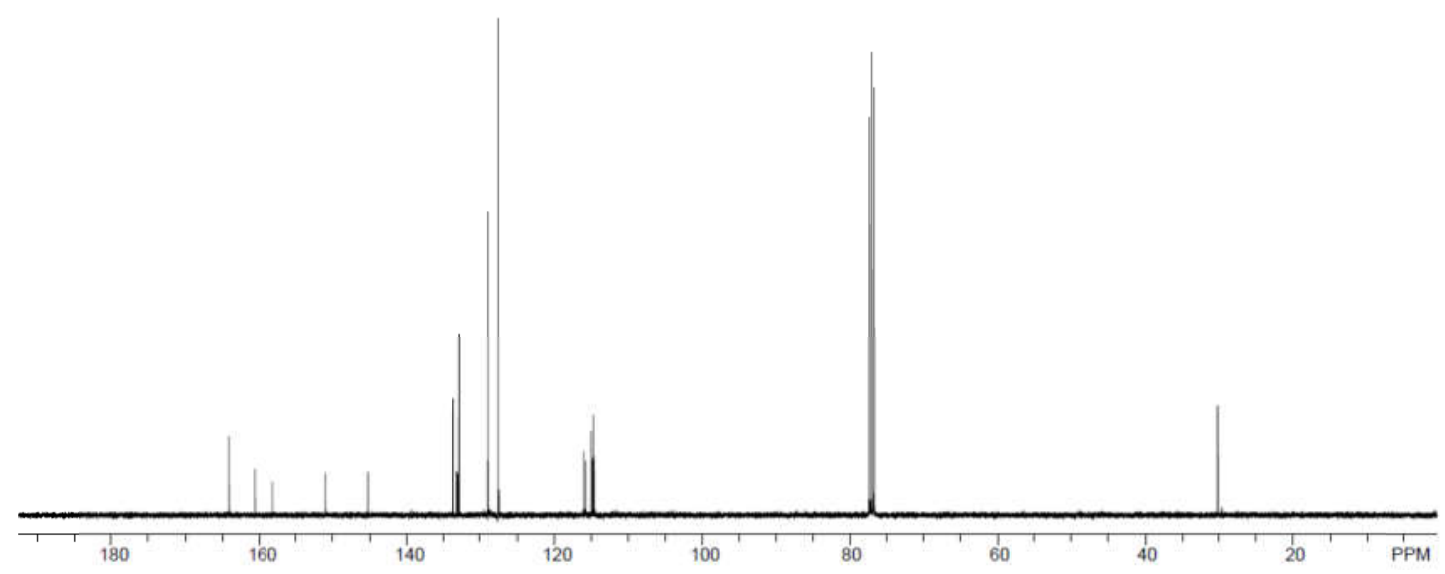

Figure S22 ${ }^{13} \mathrm{C}$ spectra of 3ka 
$N$-(7-chloro-4-methyl-3-oxo-3,4-dihydroquinoxalin-2-yl)benzamide (3la)
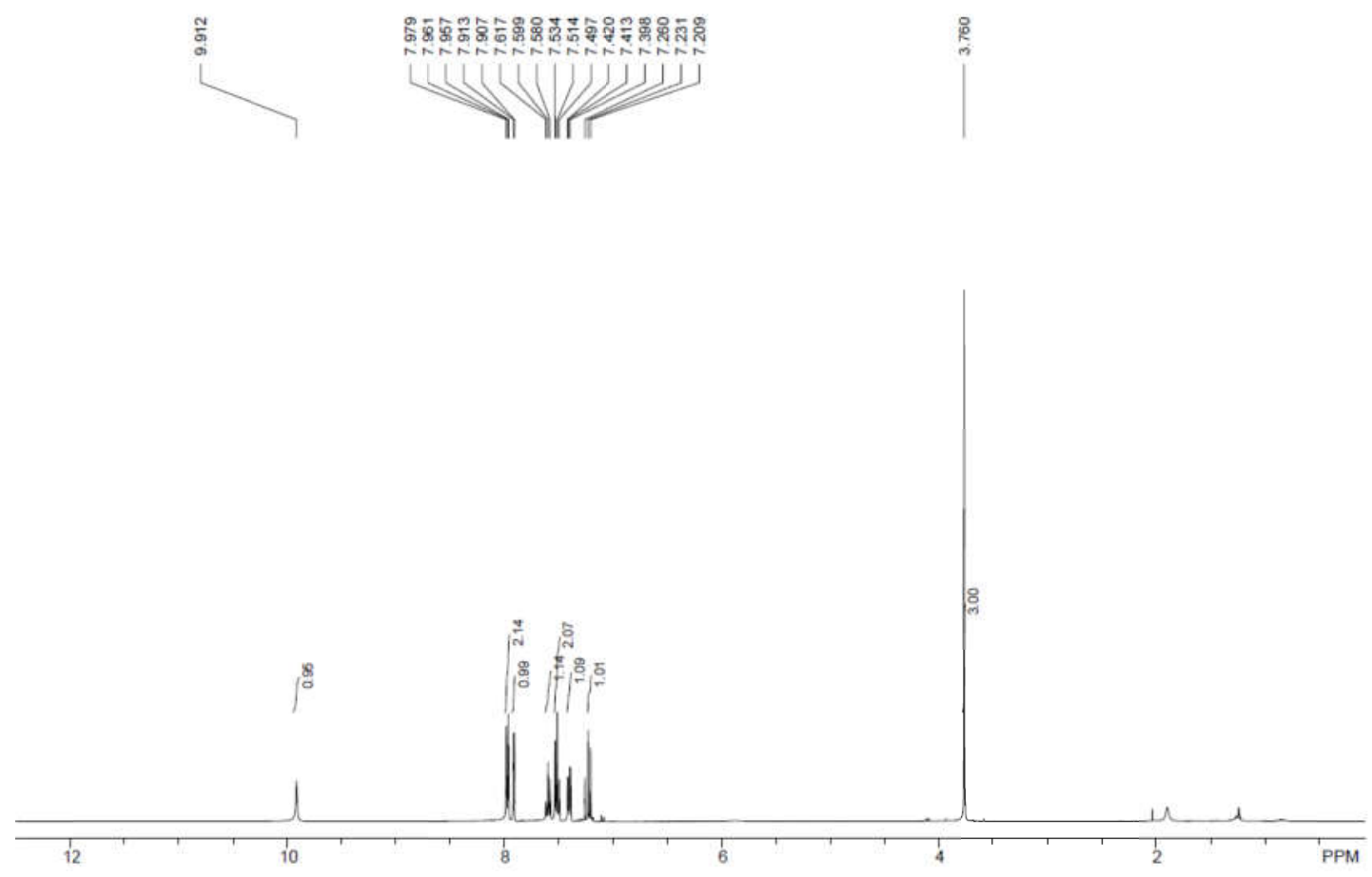

Figure $\mathbf{S 2 3}{ }^{1} \mathrm{H}$ spectra of 3la

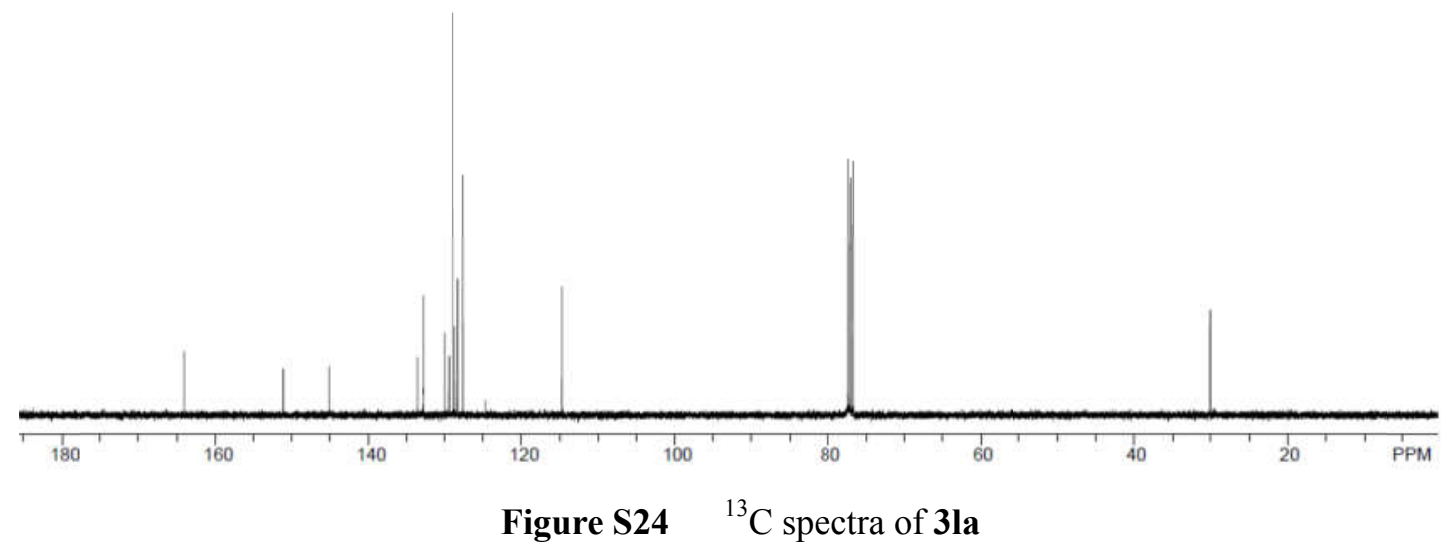


$\mathrm{N}$-(7-bromo-4-methyl-3-oxo-3,4-dihydroquinoxalin-2-yl)benzamide (3ma)
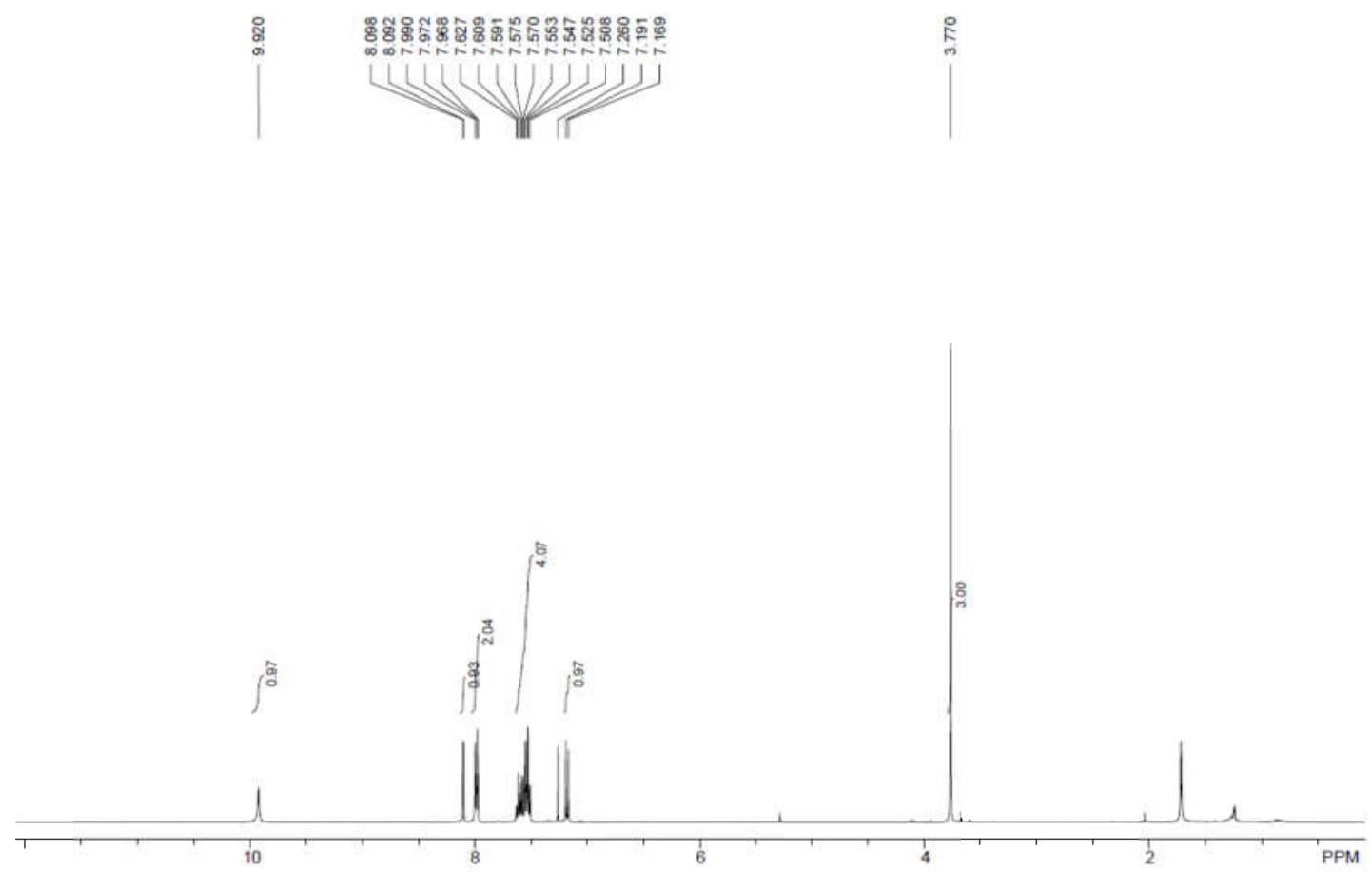

Figure S25 ${ }^{1} \mathrm{H}$ spectra of $\mathbf{3 m a}$

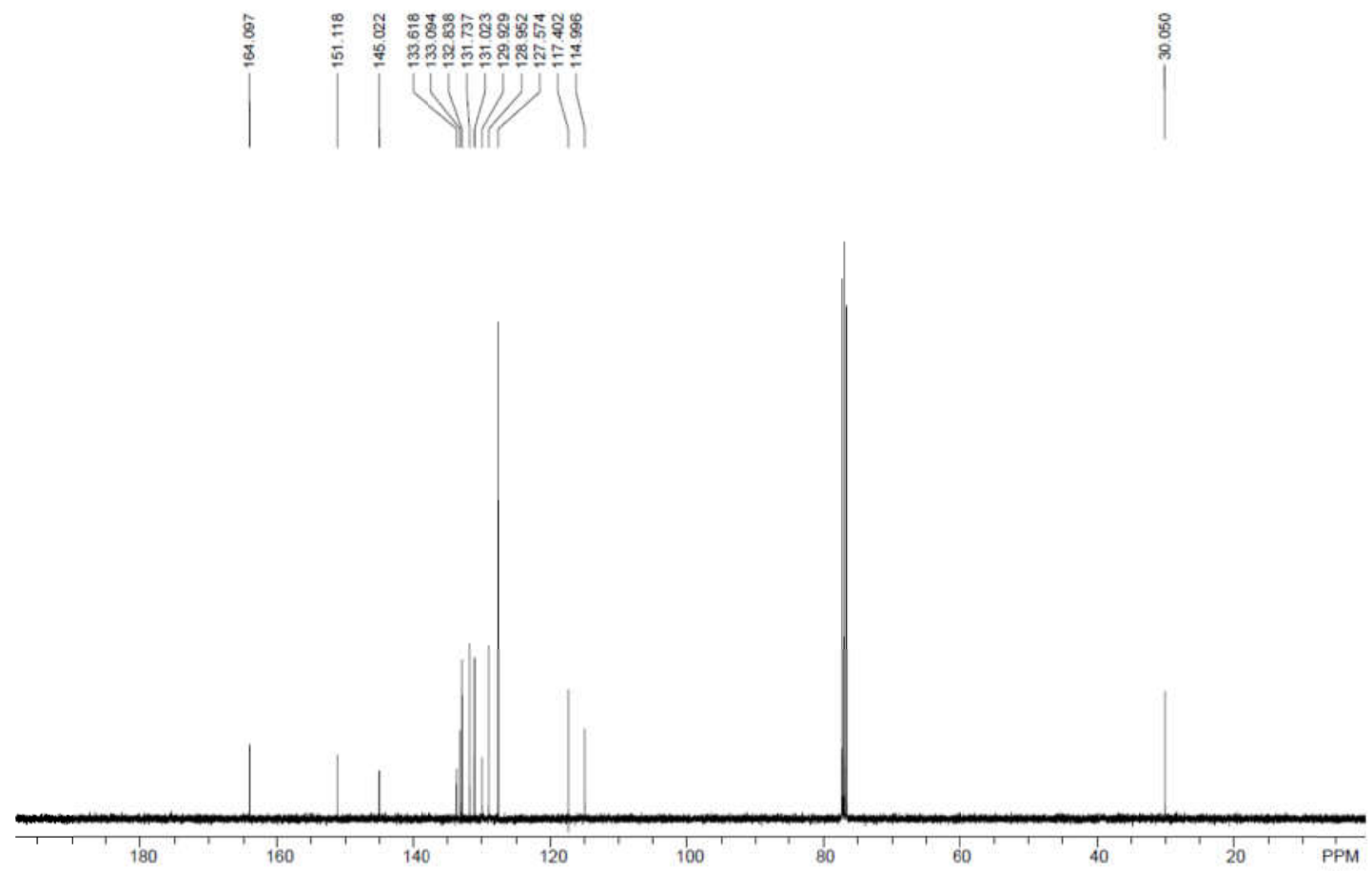

Figure S26 $\quad{ }^{13}$ Cspectra of $\mathbf{3 m a}$ 
$N$-(4-methyl-3-oxo-7-(trifluoromethyl)-3,4-dihydroquinoxalin-2-yl)benzamide (3na)
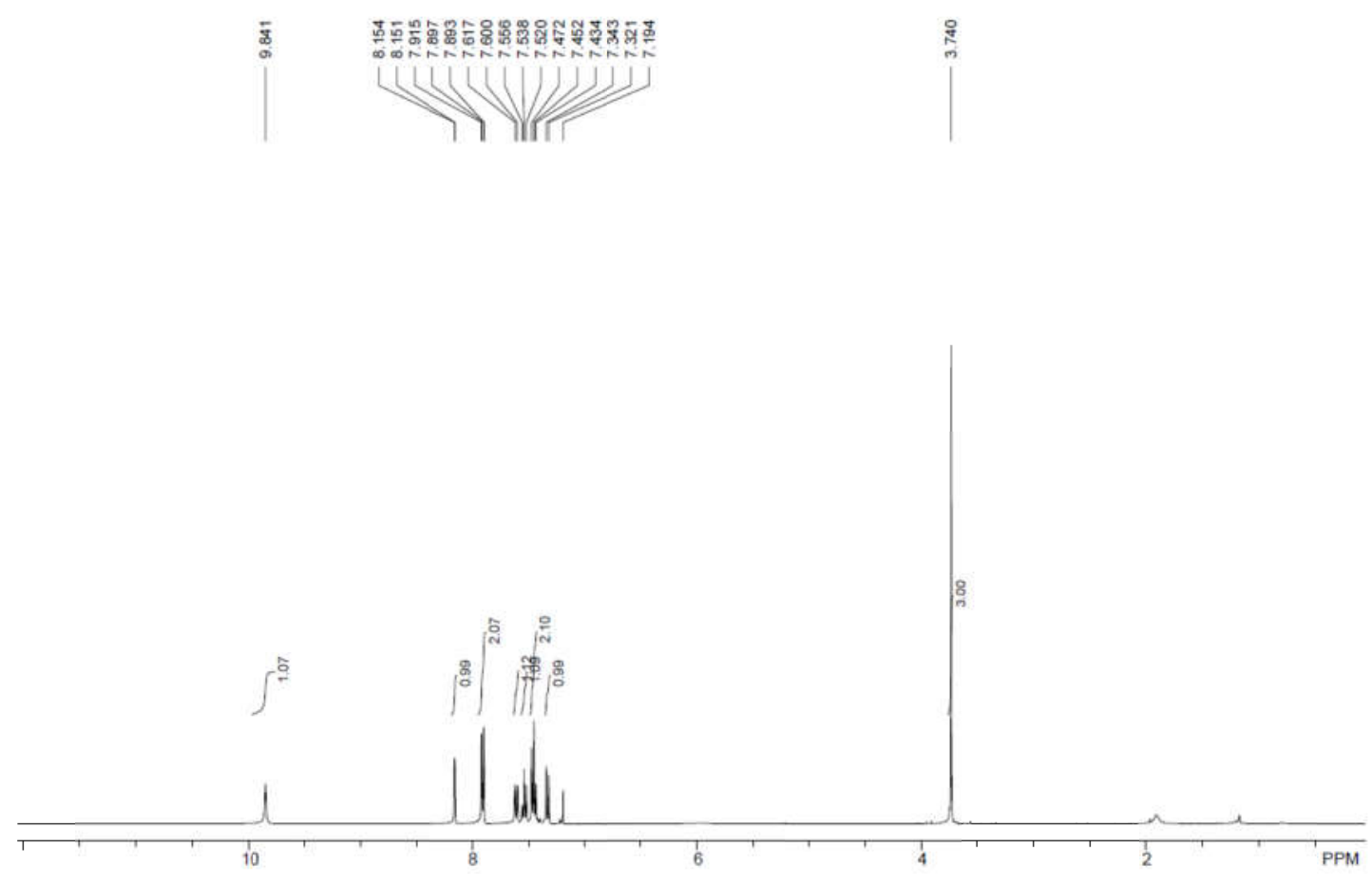

Figure S27 $\quad{ }^{1} \mathrm{H}$ spectra of $\mathbf{3 n a}$

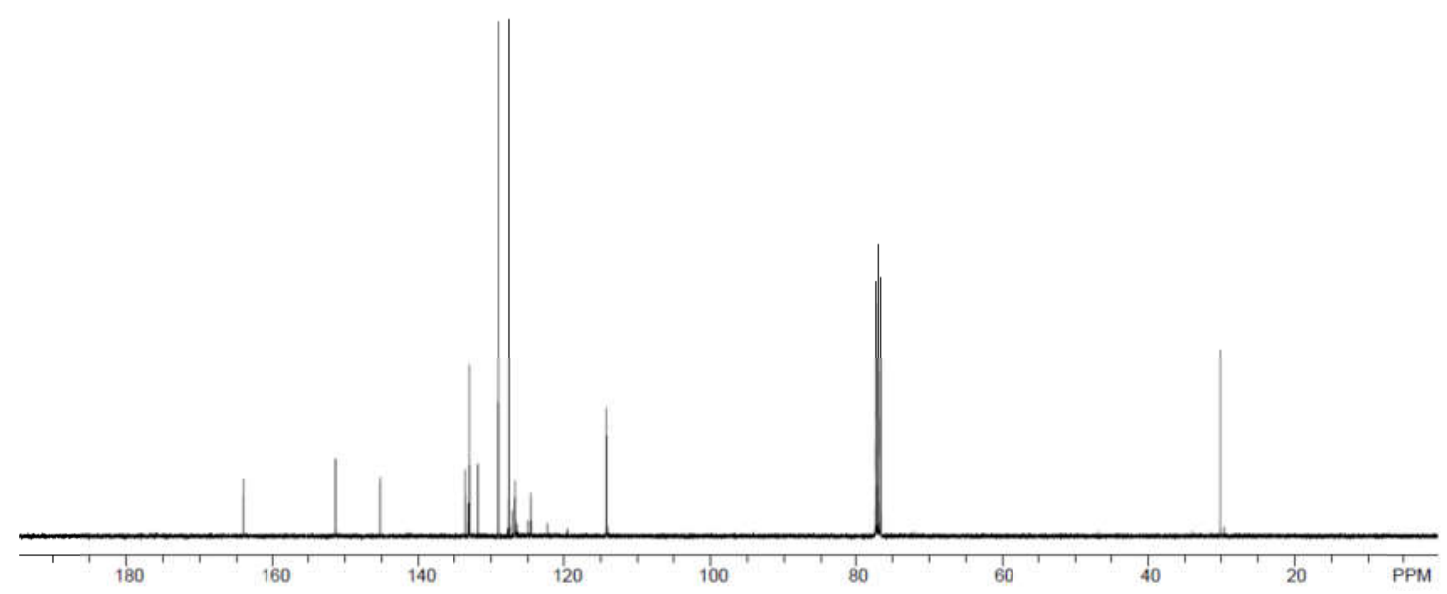

Figure S28 $\quad{ }^{13} \mathrm{C}$ spectra of 3na 
$N$-(4-methyl-3-oxo-6-(trifluoromethyl)-3,4-dihydroquinoxalin-2-yl)benzamide (3oa)

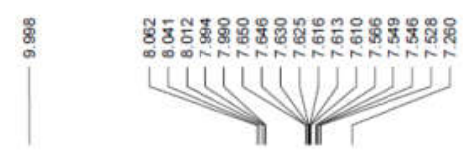

管
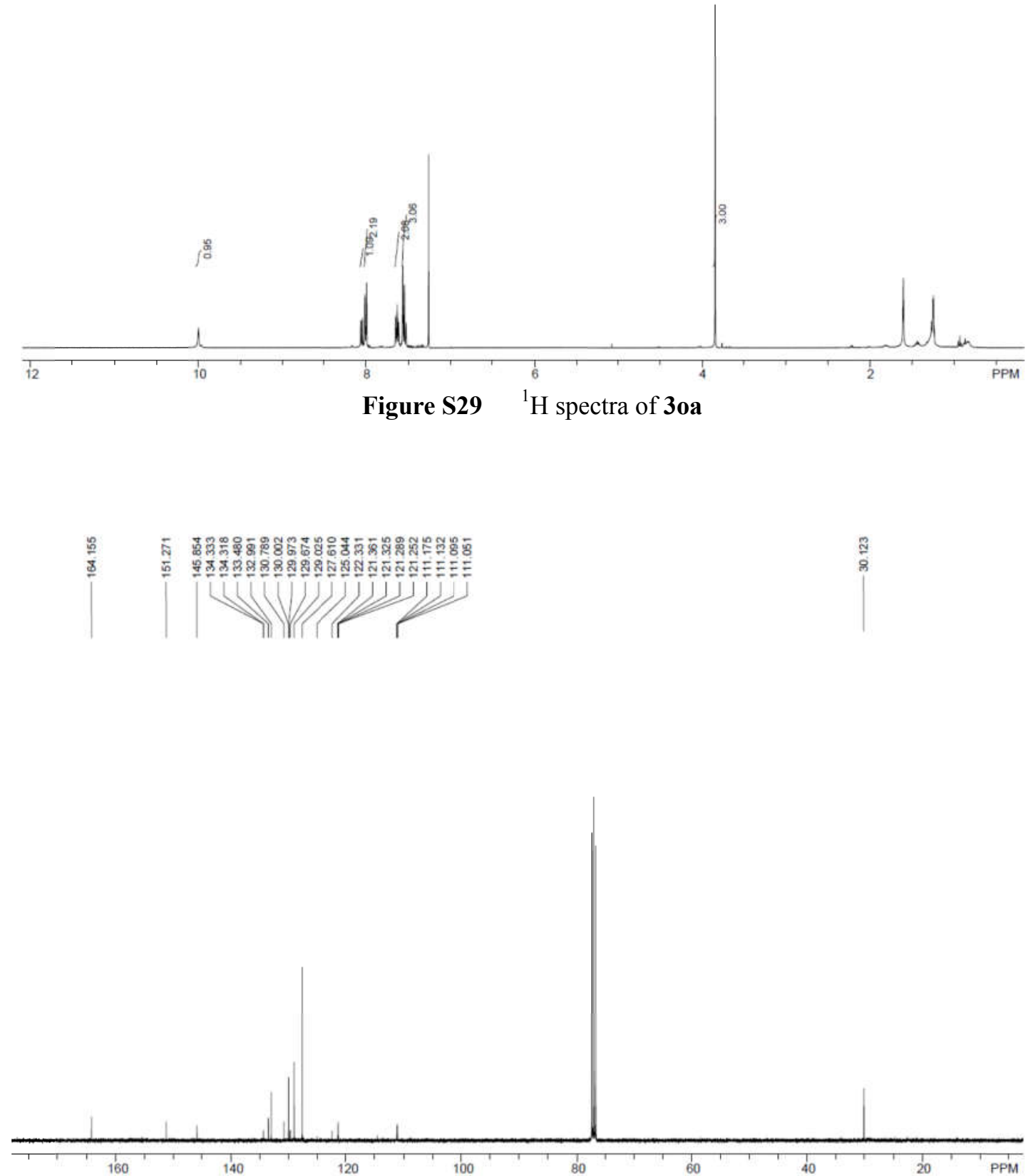

Figure S30 ${ }^{13} \mathrm{C}$ spectra of $\mathbf{3 0 a}$ 
$N$-(6,7-difluoro-4-methyl-3-oxo-3,4-dihydroquinoxalin-2-yl)benzamide (3pa)
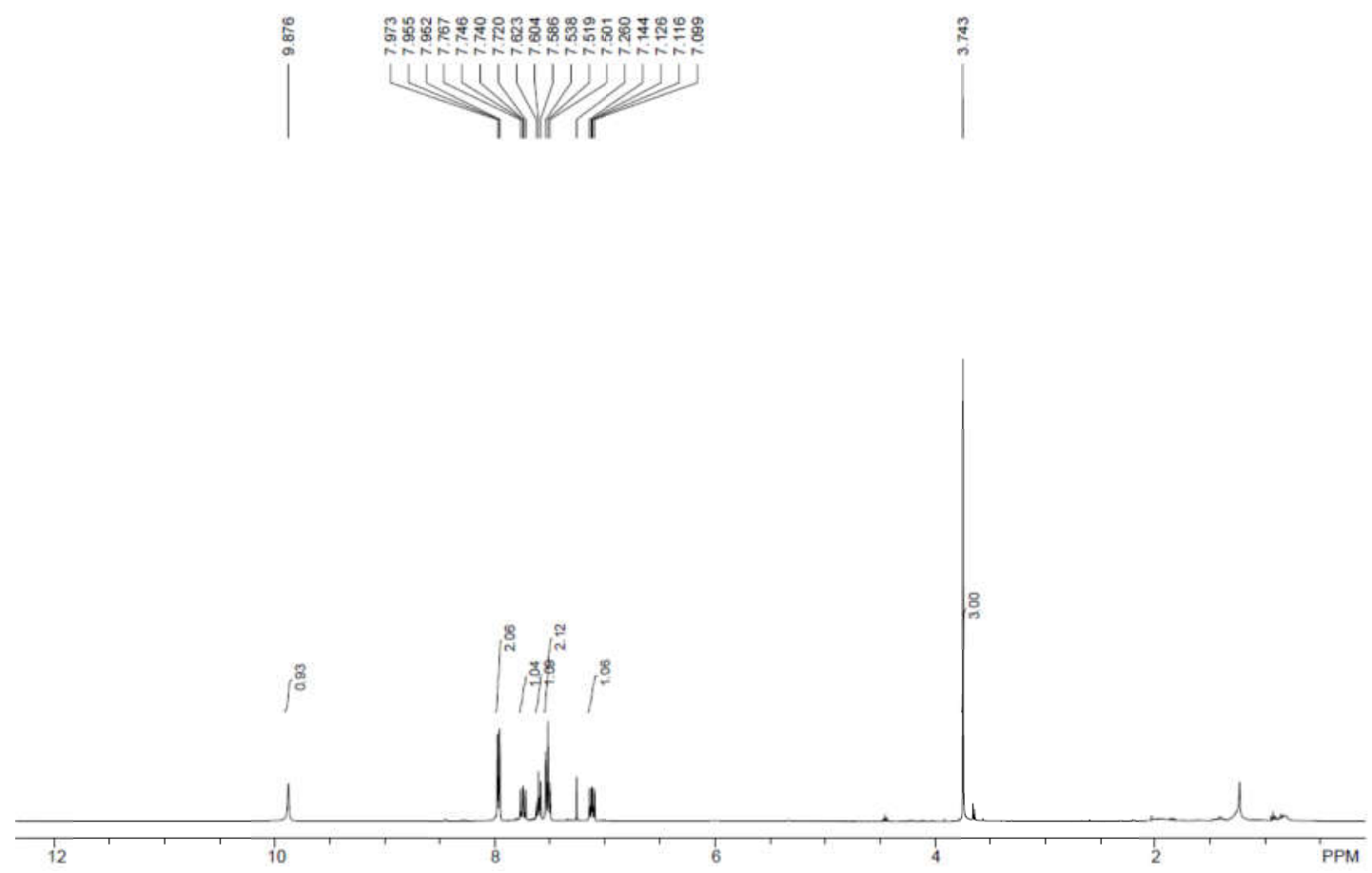

Figure S31 ${ }^{1} \mathrm{H}$ spectra of $\mathbf{3 p a}$

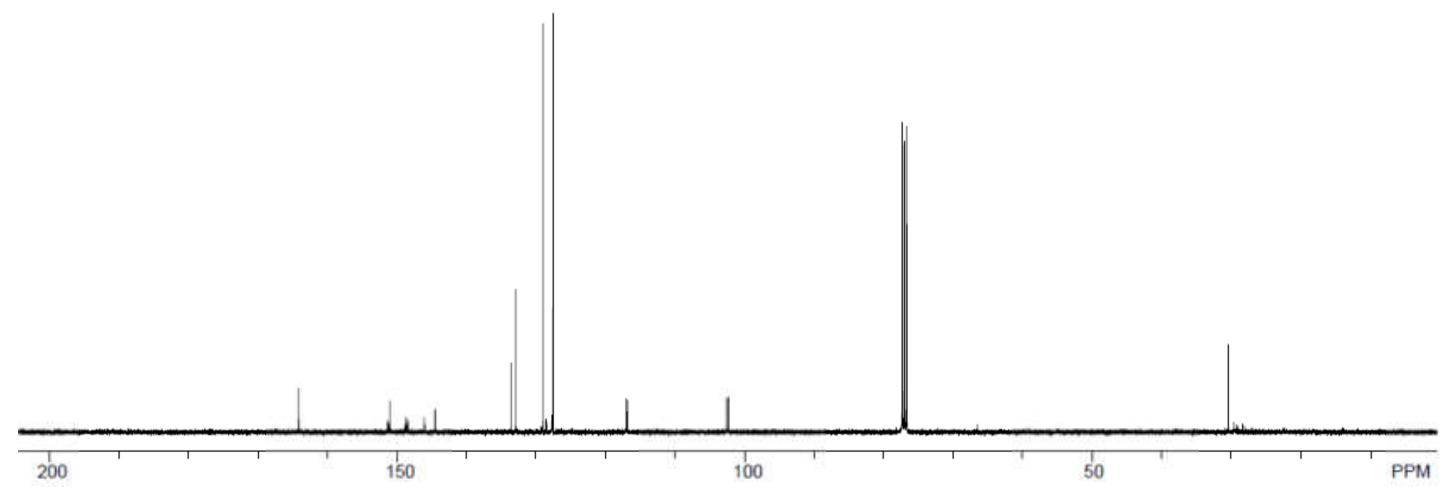

Figure S32 ${ }^{13} \mathrm{C}$ spectra of $\mathbf{3 p a}$ 
$N$-(4-methyl-3-oxo-3,4-dihydrobenzo[g]quinoxalin-2-yl)benzamide (3qa)
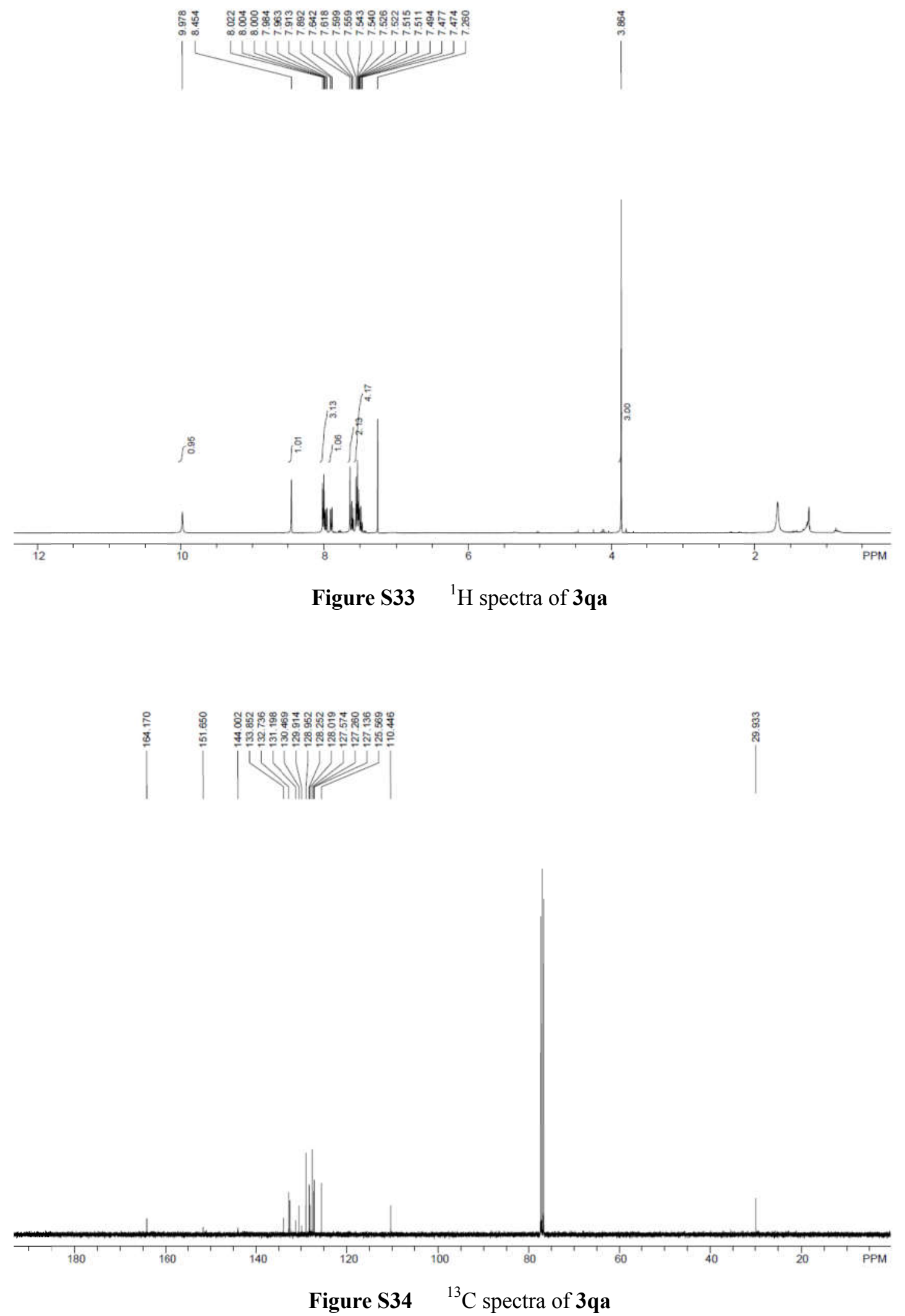
4-methyl-N-(3-oxo-4-pentyl-3,4-dihydroquinoxalin-2-yl)benzamide (3ab)

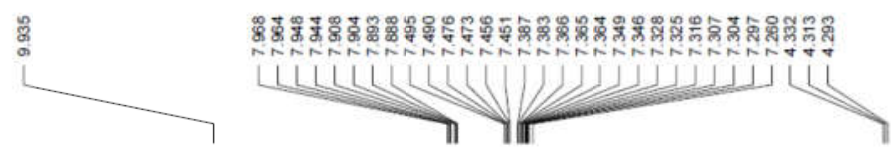

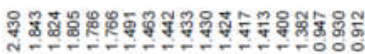
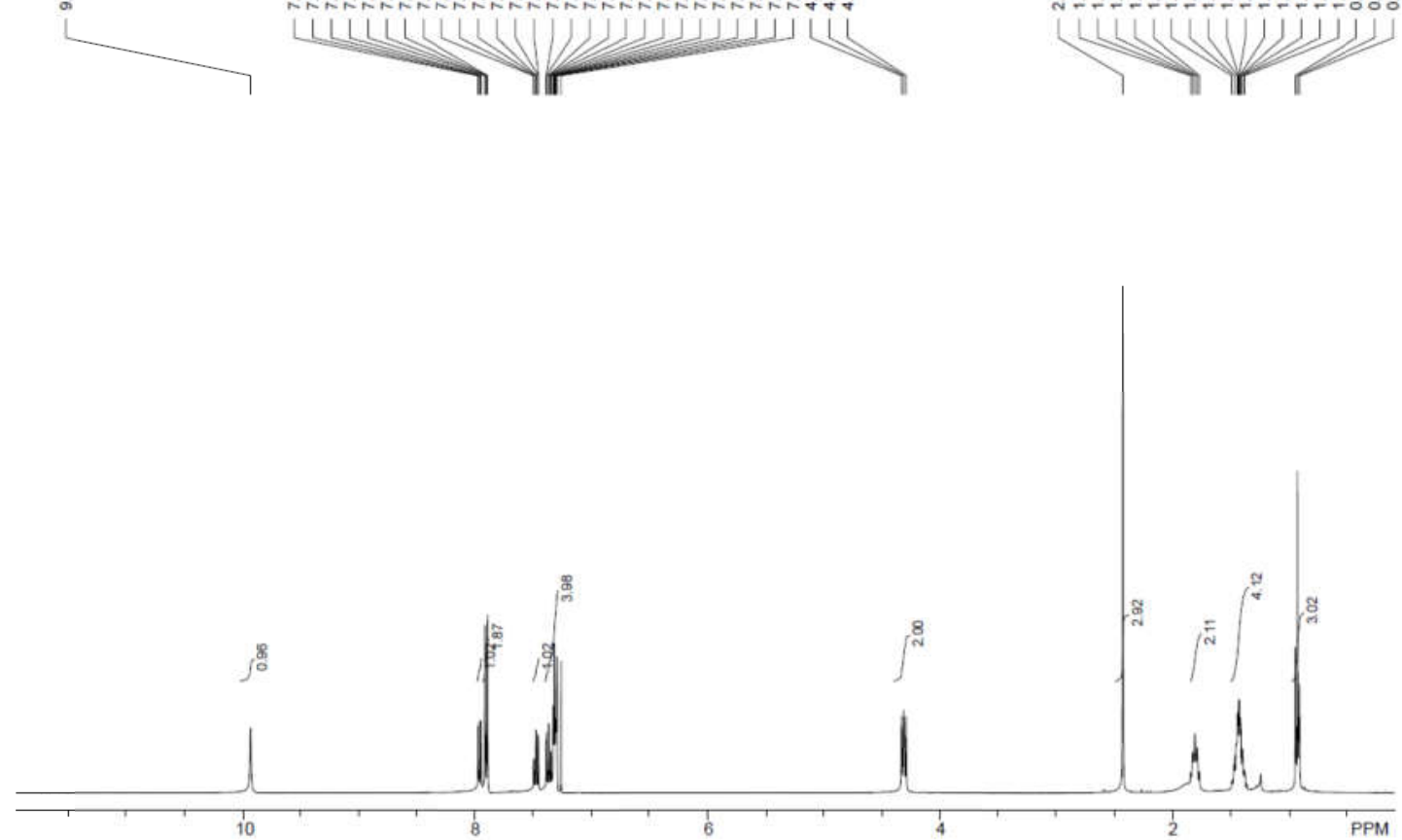

Figure S35 $\quad{ }^{1} \mathrm{H}$ spectra of $\mathbf{3 a b}$
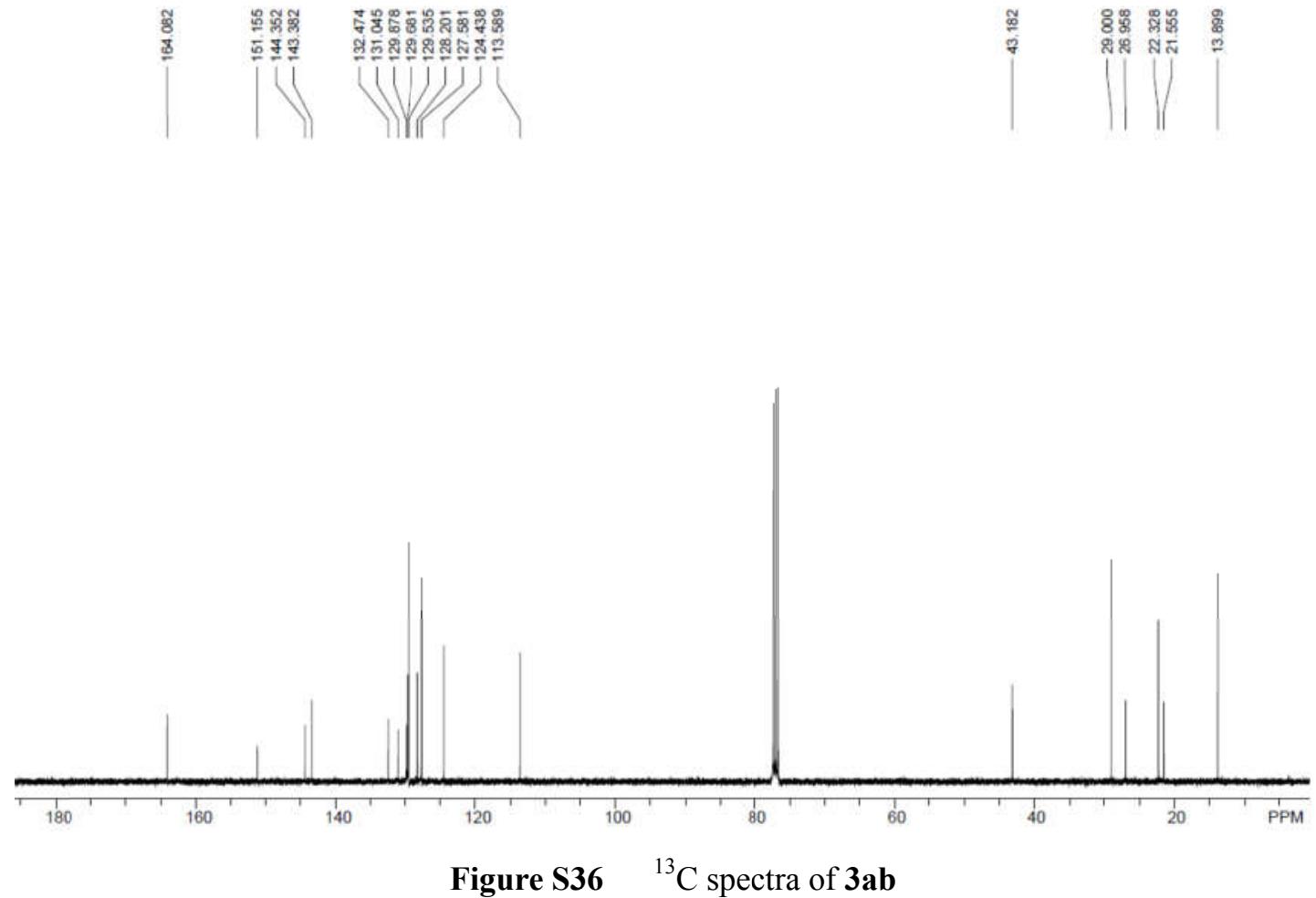
4-(tert-butyl)-N-(3-oxo-4-pentyl-3,4-dihydroquinoxalin-2-yl)benzamide (3ac)
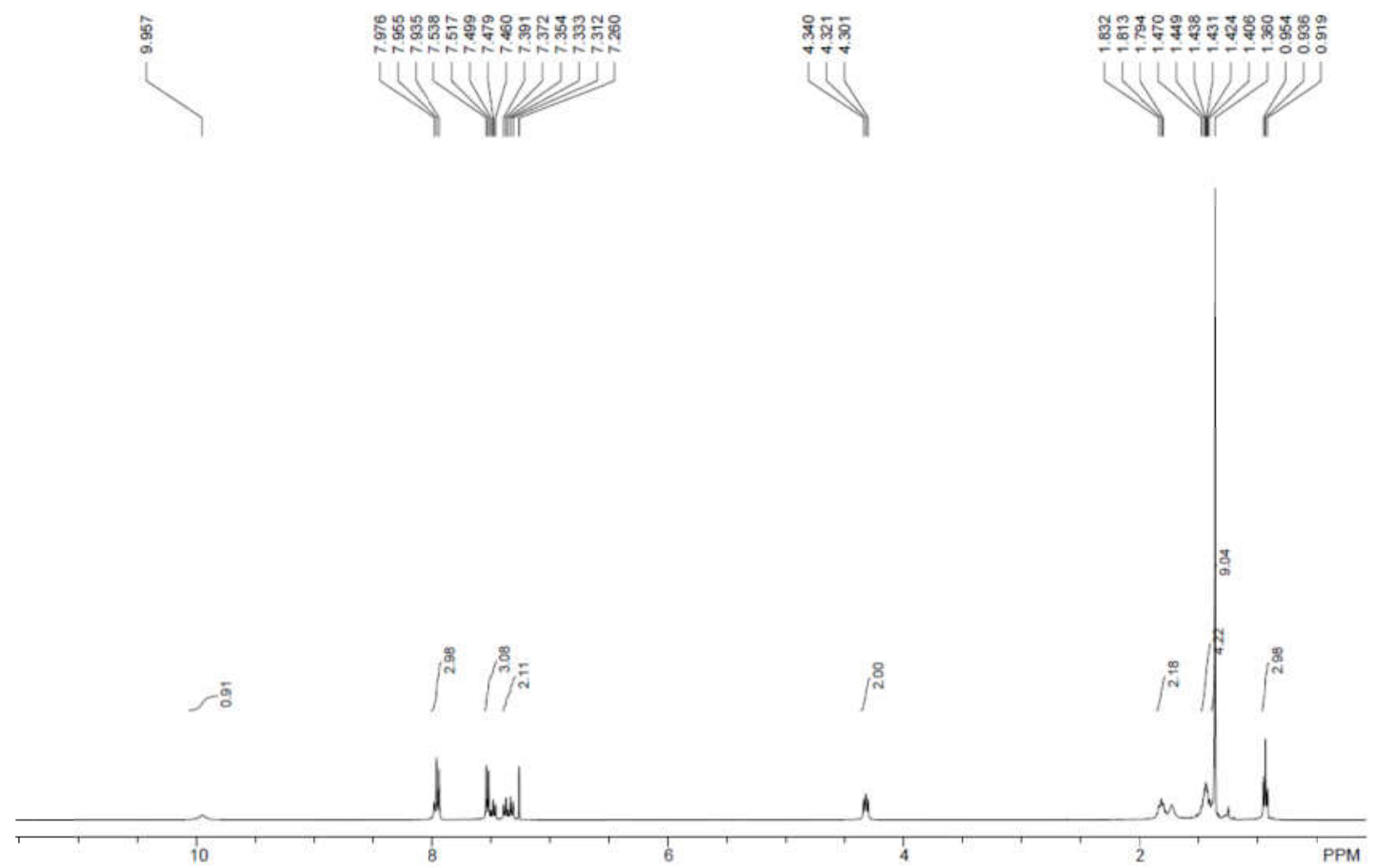

Figure S37 $\quad{ }^{1} \mathrm{H}$ spectra of 3ac

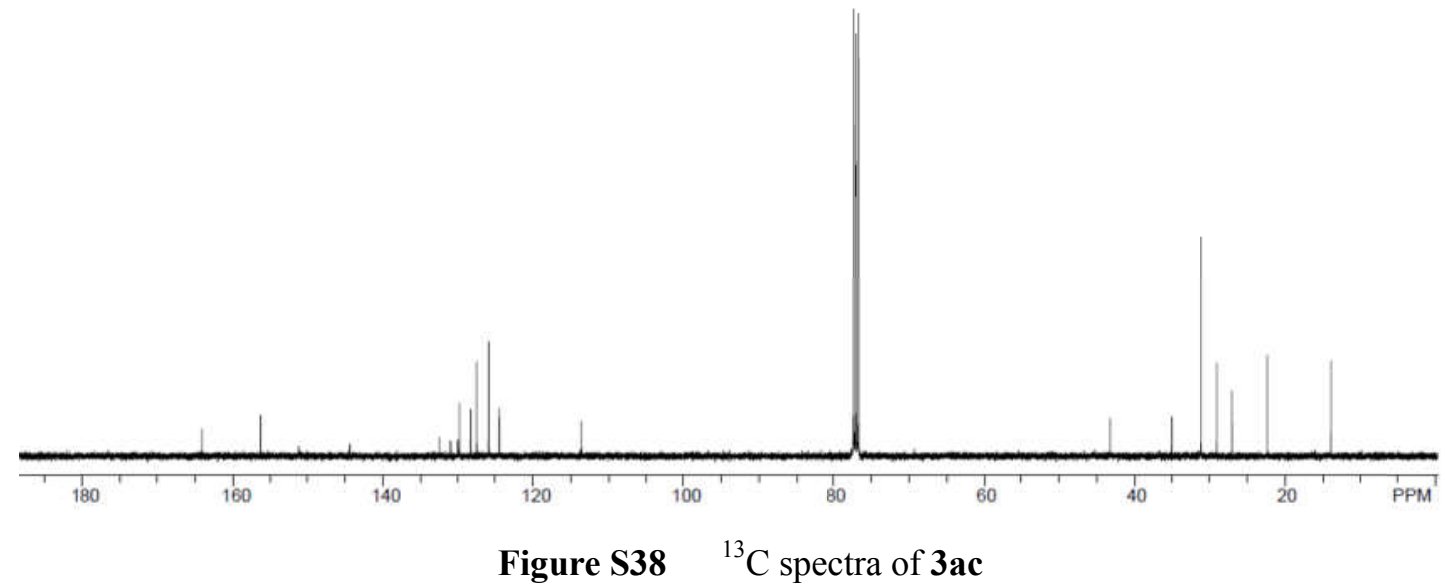


4-methoxy- $N$-(3-oxo-4-pentyl-3,4-dihydroquinoxalin-2-yl)benzamide (3ad)

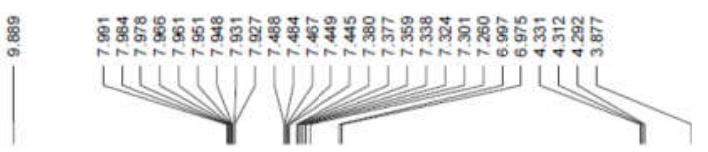

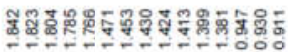
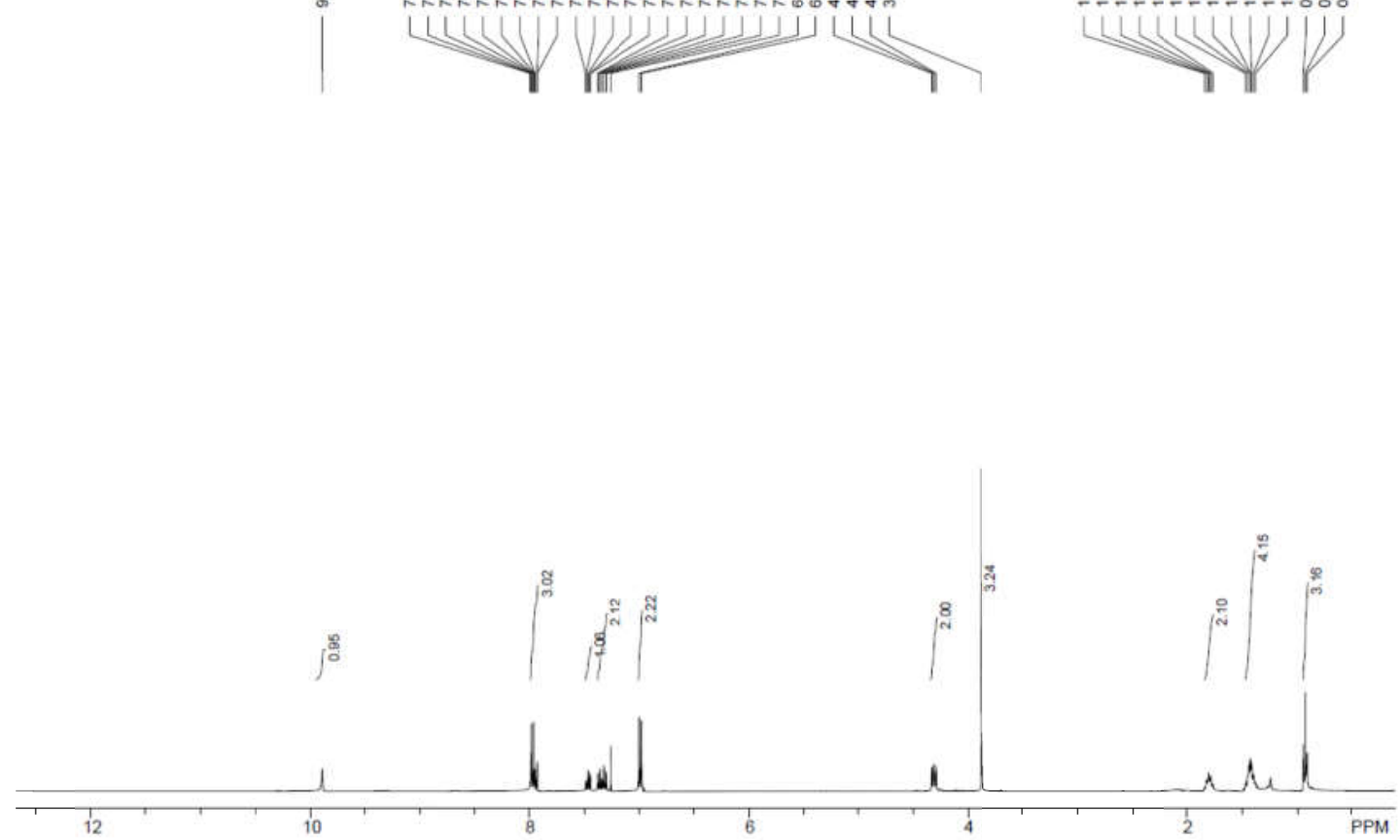

Figure S39 $\quad{ }^{1} \mathrm{H}$ spectra of $\mathbf{3 a d}$
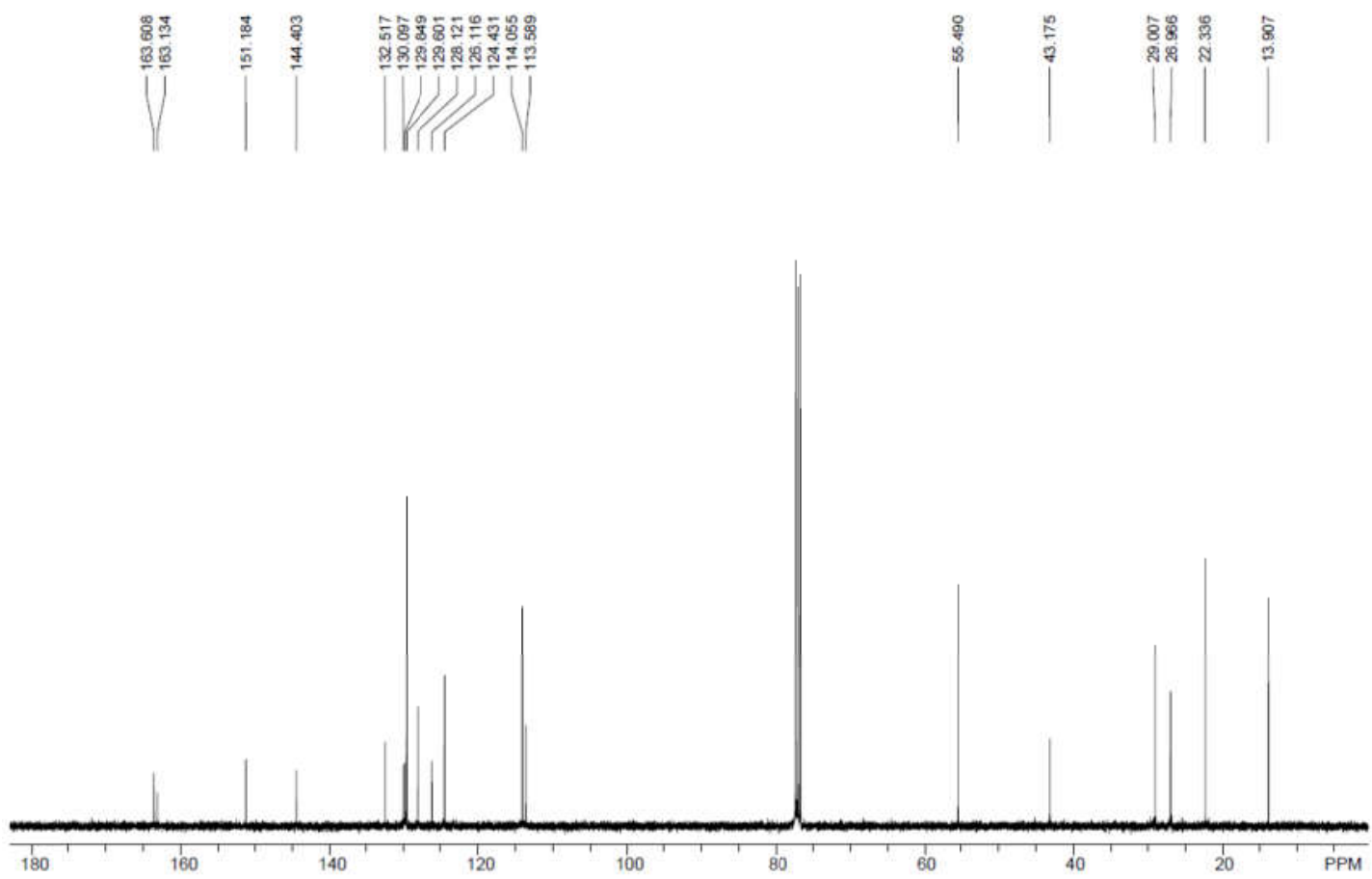

Figure $\mathbf{S 4 0}{ }^{13} \mathrm{C}$ spectra of $\mathbf{3 a d}$ 
4-fluoro- $N$-(3-oxo-4-pentyl-3,4-dihydroquinoxalin-2-yl)benzamide (3ae)

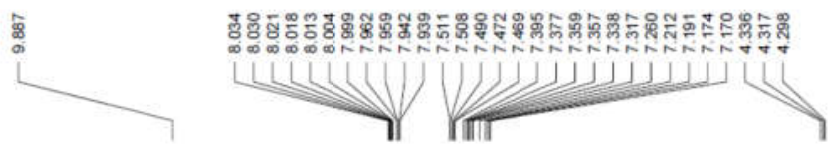

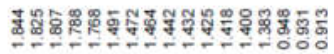
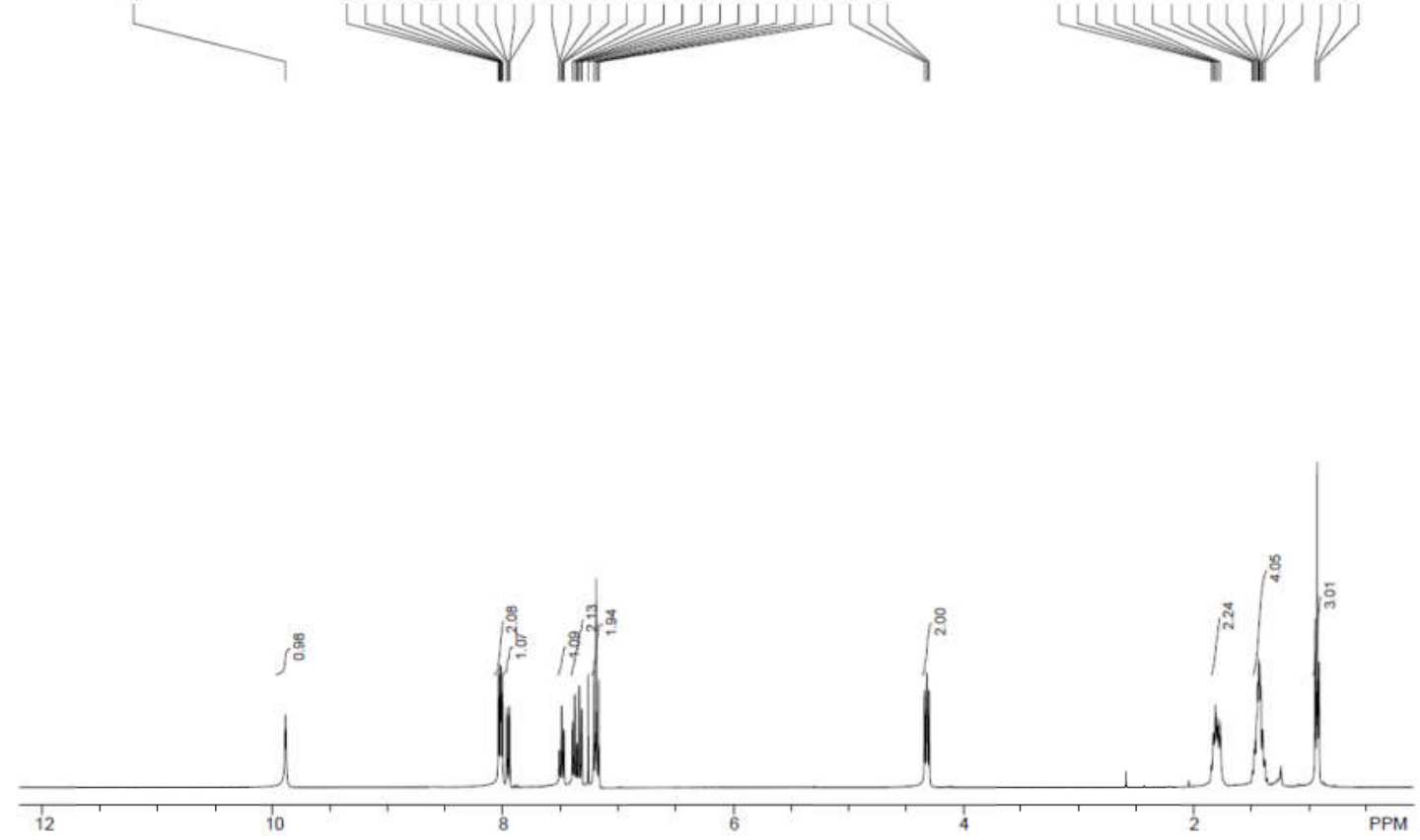

Figure S41 ${ }^{1} \mathrm{H}$ spectra of 3ae

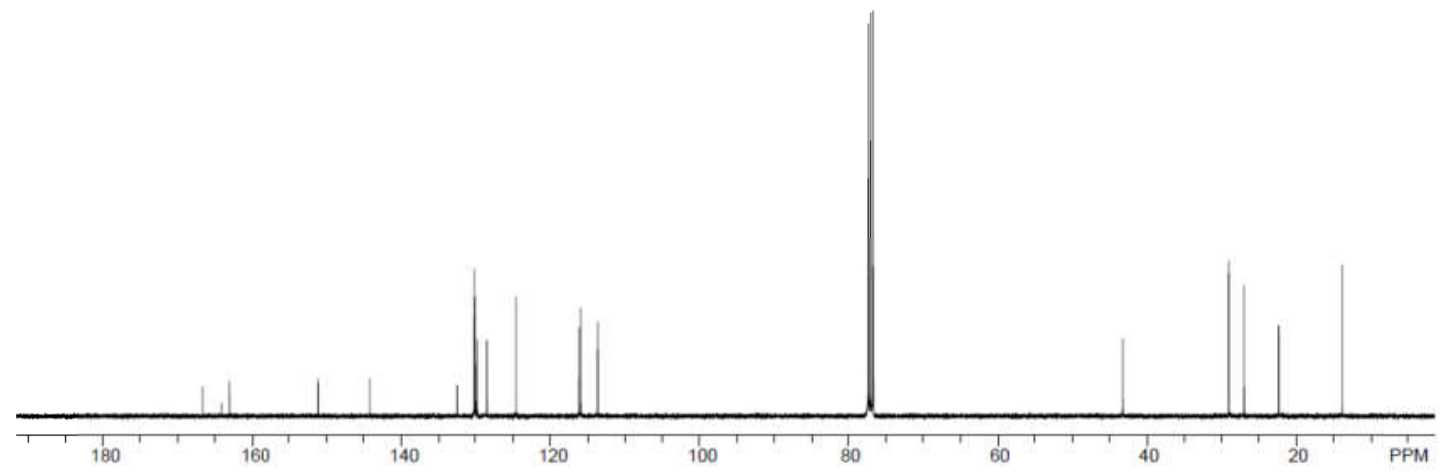

Figure $\mathbf{S 4 2}{ }^{13} \mathrm{C}$ spectra of 3ae 
4-chloro-N-(3-oxo-4-pentyl-3,4-dihydroquinoxalin-2-yl)benzamide (3af)
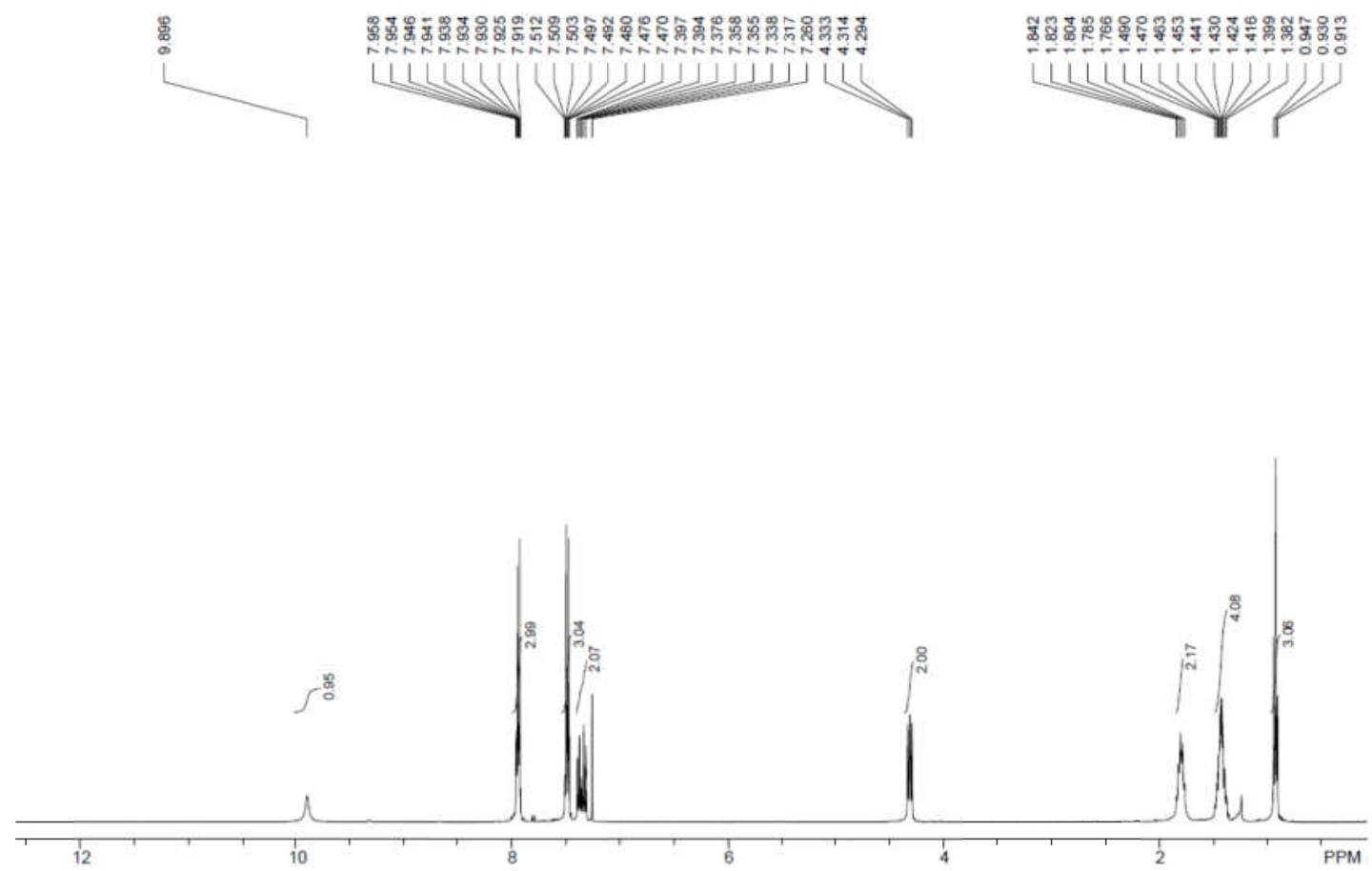

Figure $\mathbf{S 4 3}{ }^{1} \mathrm{H}$ spectra of $\mathbf{3 a f}$

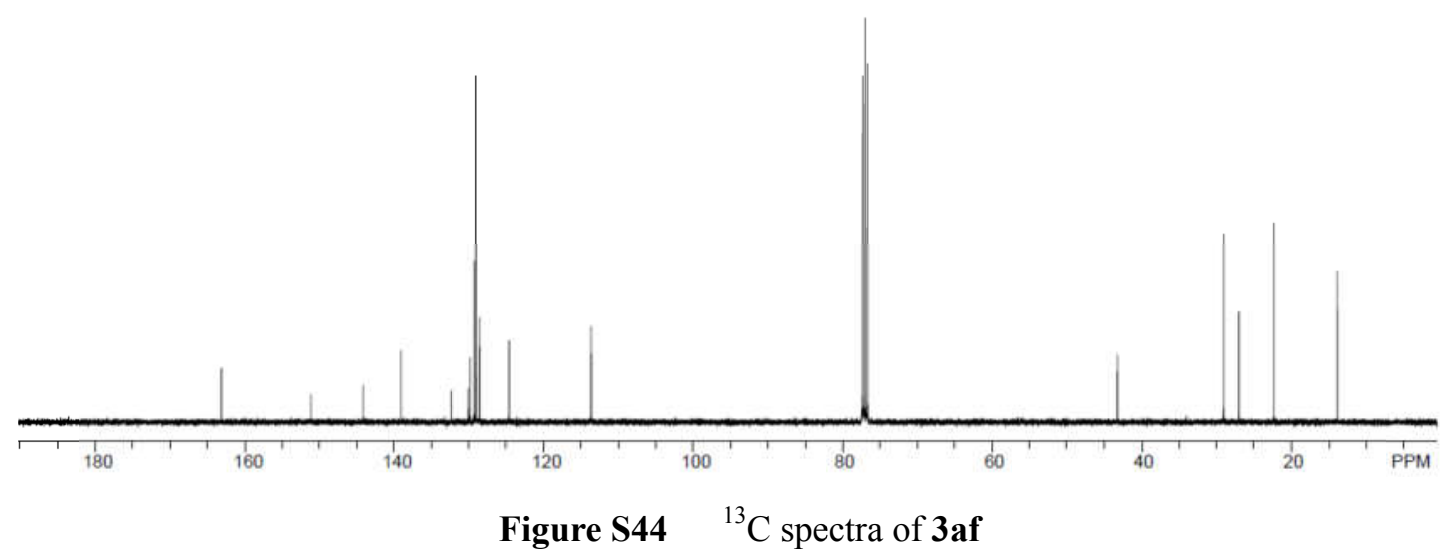


4-bromo- $N$-(3-oxo-4-pentyl-3,4-dihydroquinoxalin-2-yl)benzamide (3ag)
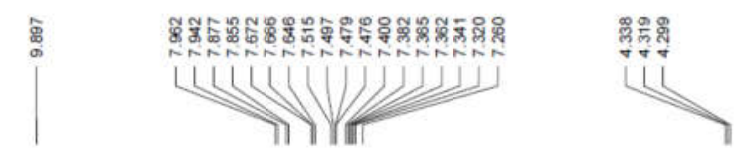

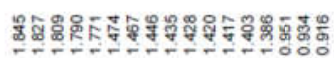
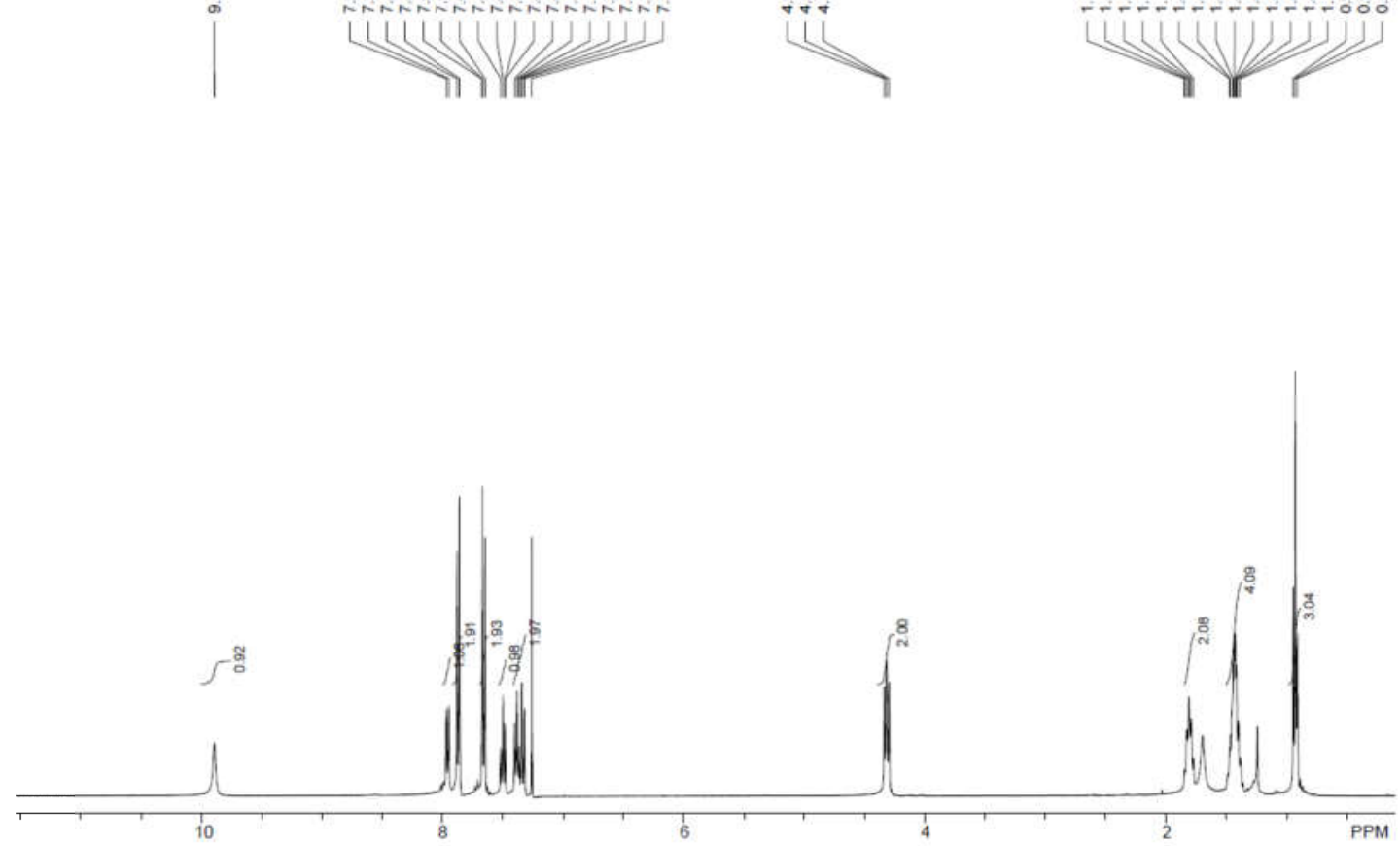

Figure S45 $\quad{ }^{1} \mathrm{H}$ spectra of $\mathbf{3 a g}$
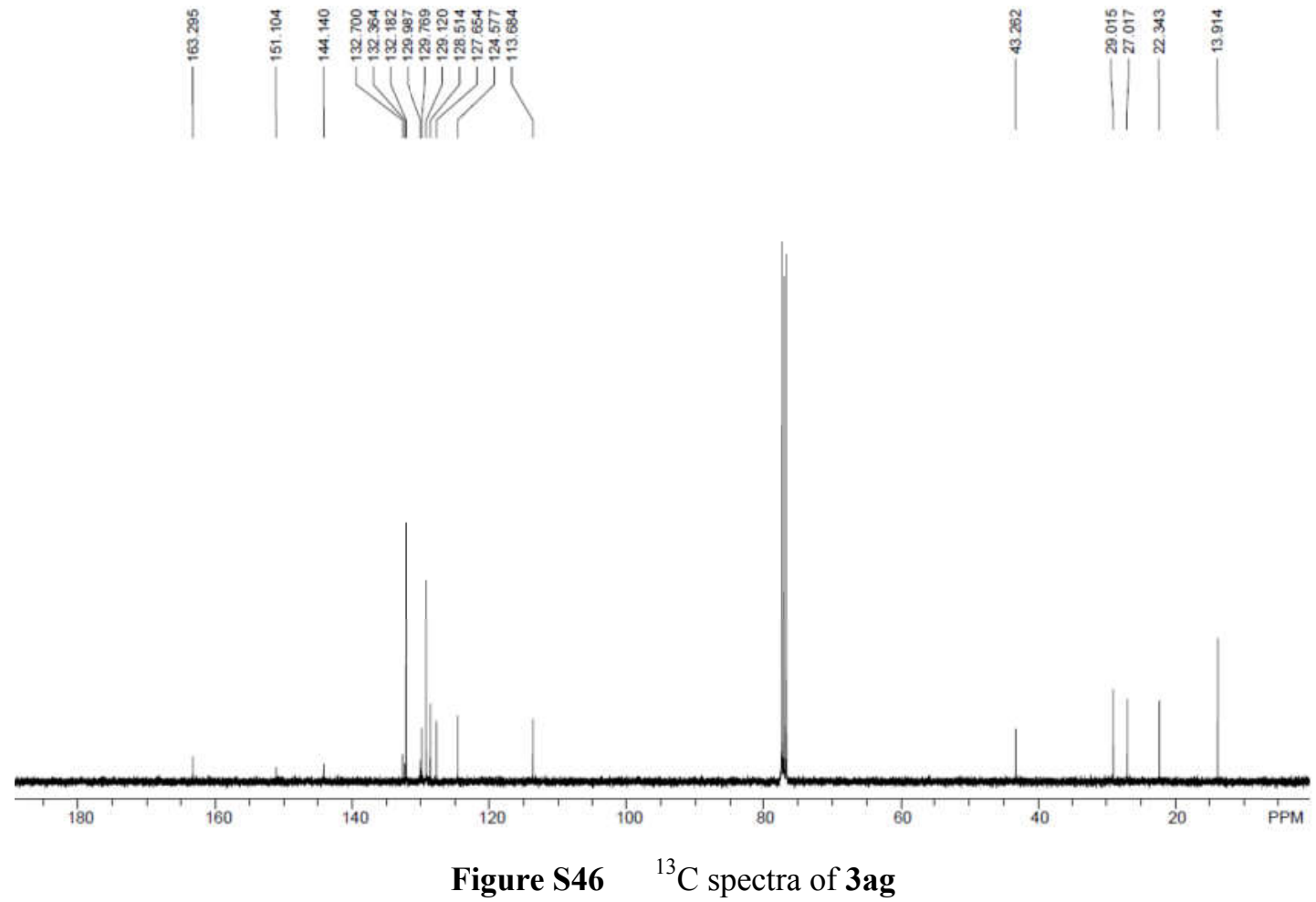
$N$-(3-oxo-4-pentyl-3,4-dihydroquinoxalin-2-yl)-4-(trifluoromethyl)benzamide (3ah)
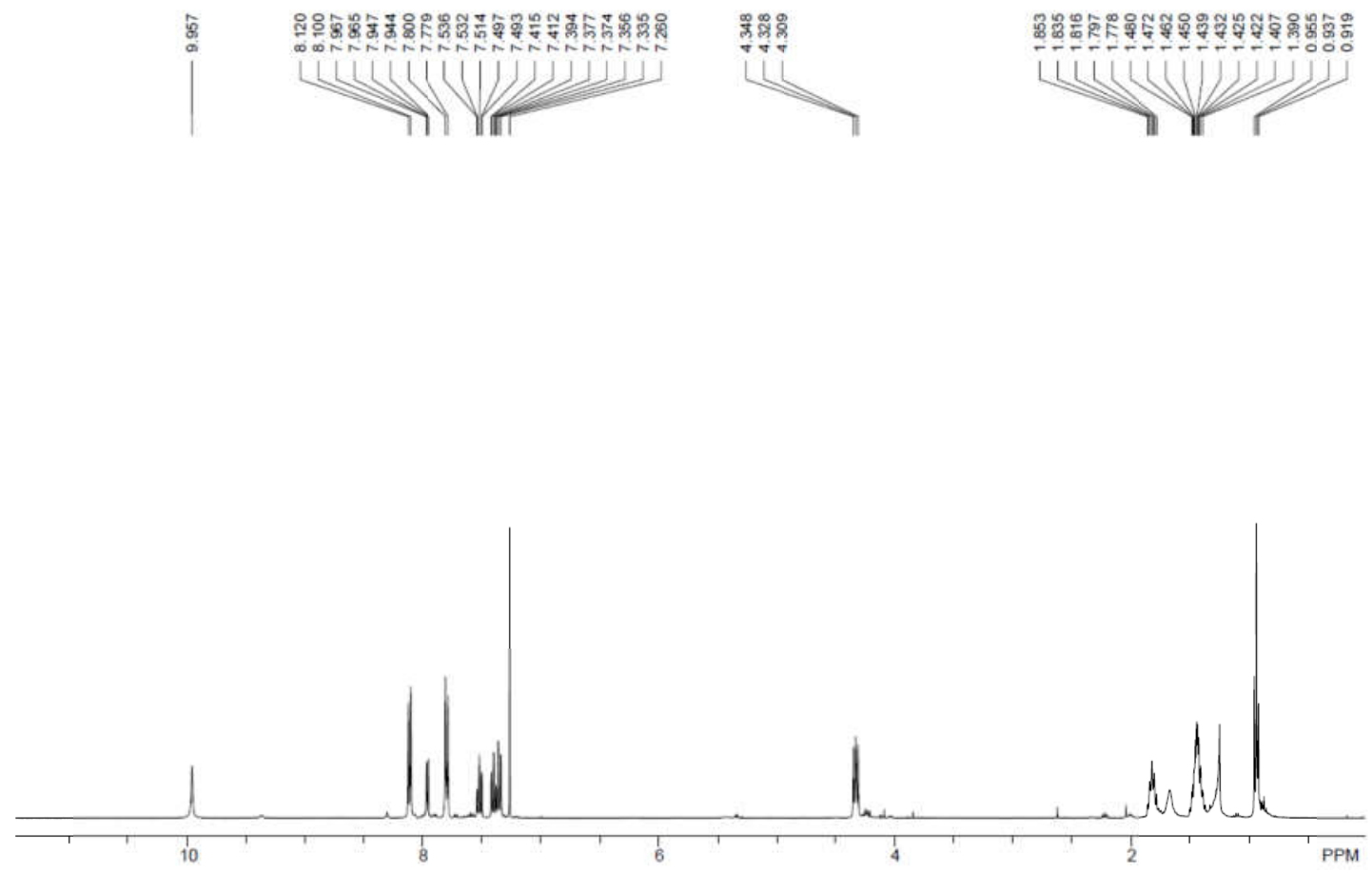

Figure $\mathbf{S 4 7} \quad{ }^{1} \mathrm{H}$ spectra of $\mathbf{3 a h}$

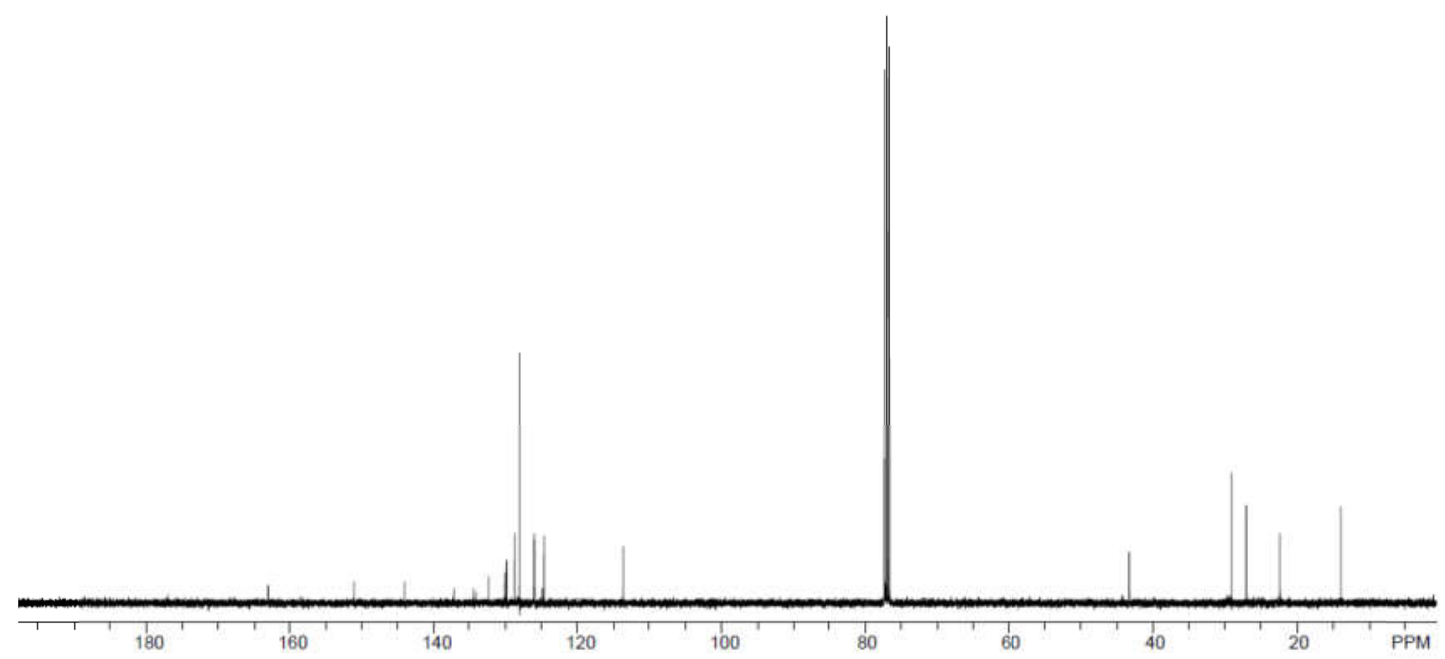

Figure $\mathbf{S 4 8}{ }^{13} \mathrm{C}$ spectra of $\mathbf{3 a h}$ 
4-nitro- $N$-(3-oxo-4-pentyl-3,4-dihydroquinoxalin-2-yl)benzamide (3ai)
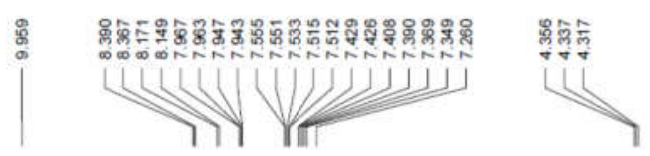

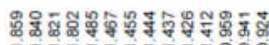
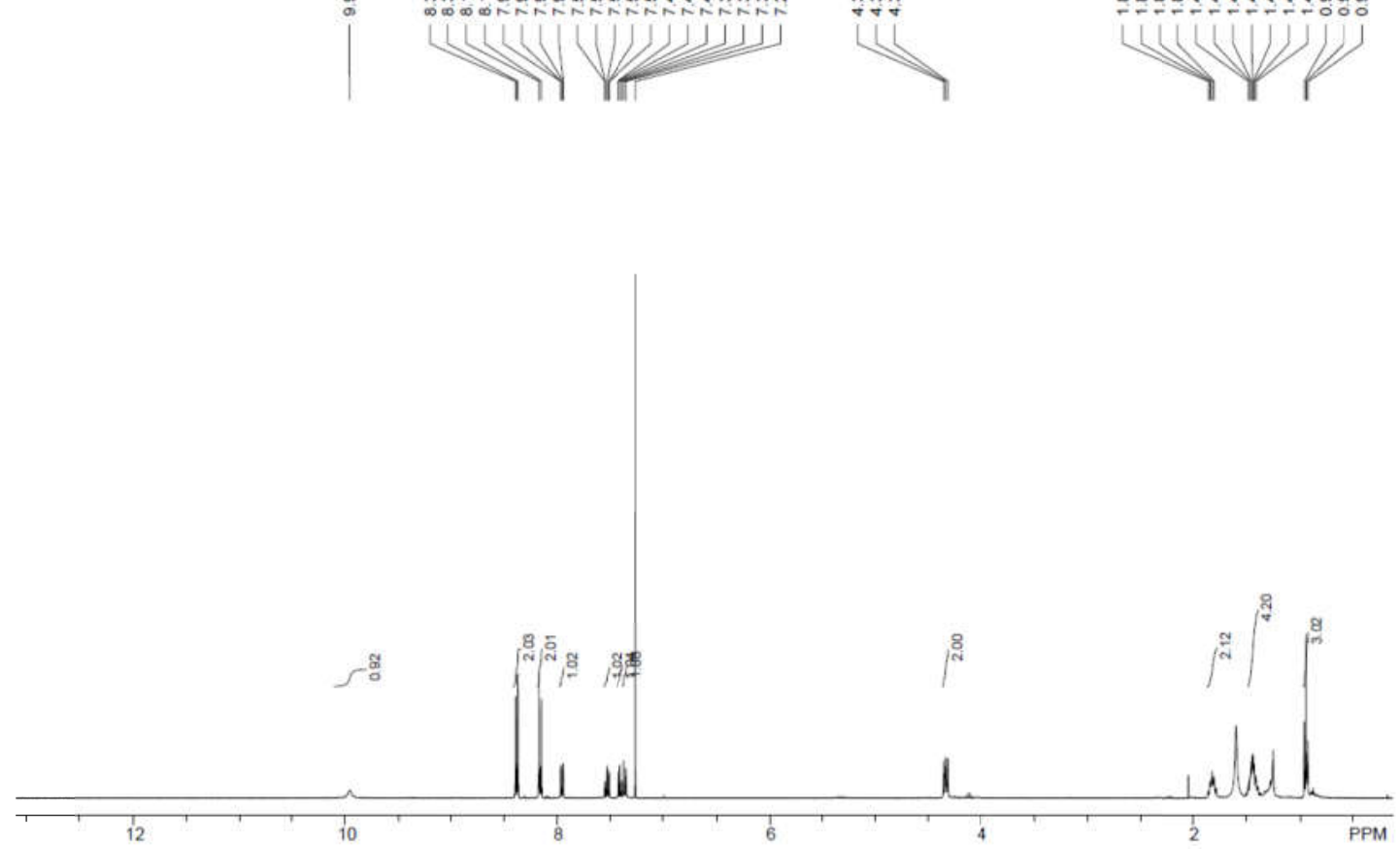

Figure $\mathbf{S 4 9}{ }^{1} \mathrm{H}$ spectra of $\mathbf{3 a i}$

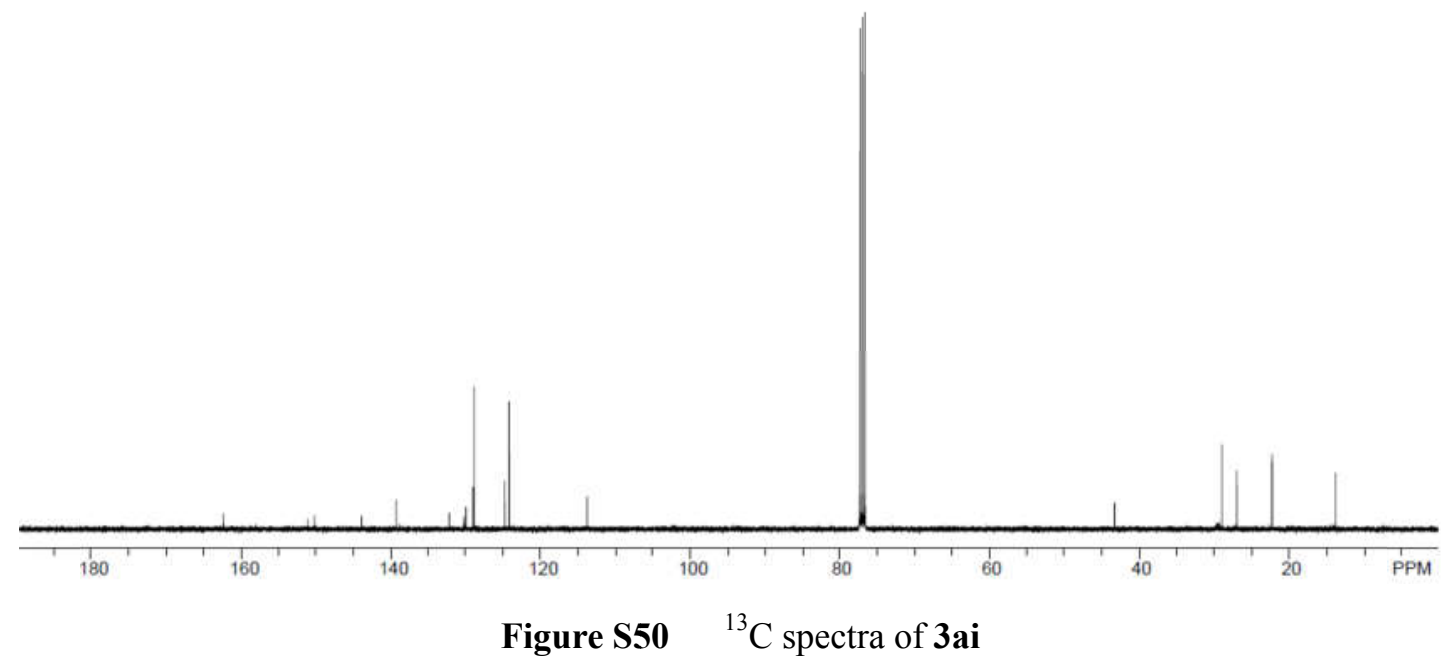


$N$-(3-oxo-4-pentyl-3,4-dihydroquinoxalin-2-yl)-2-naphthamide (3aj)
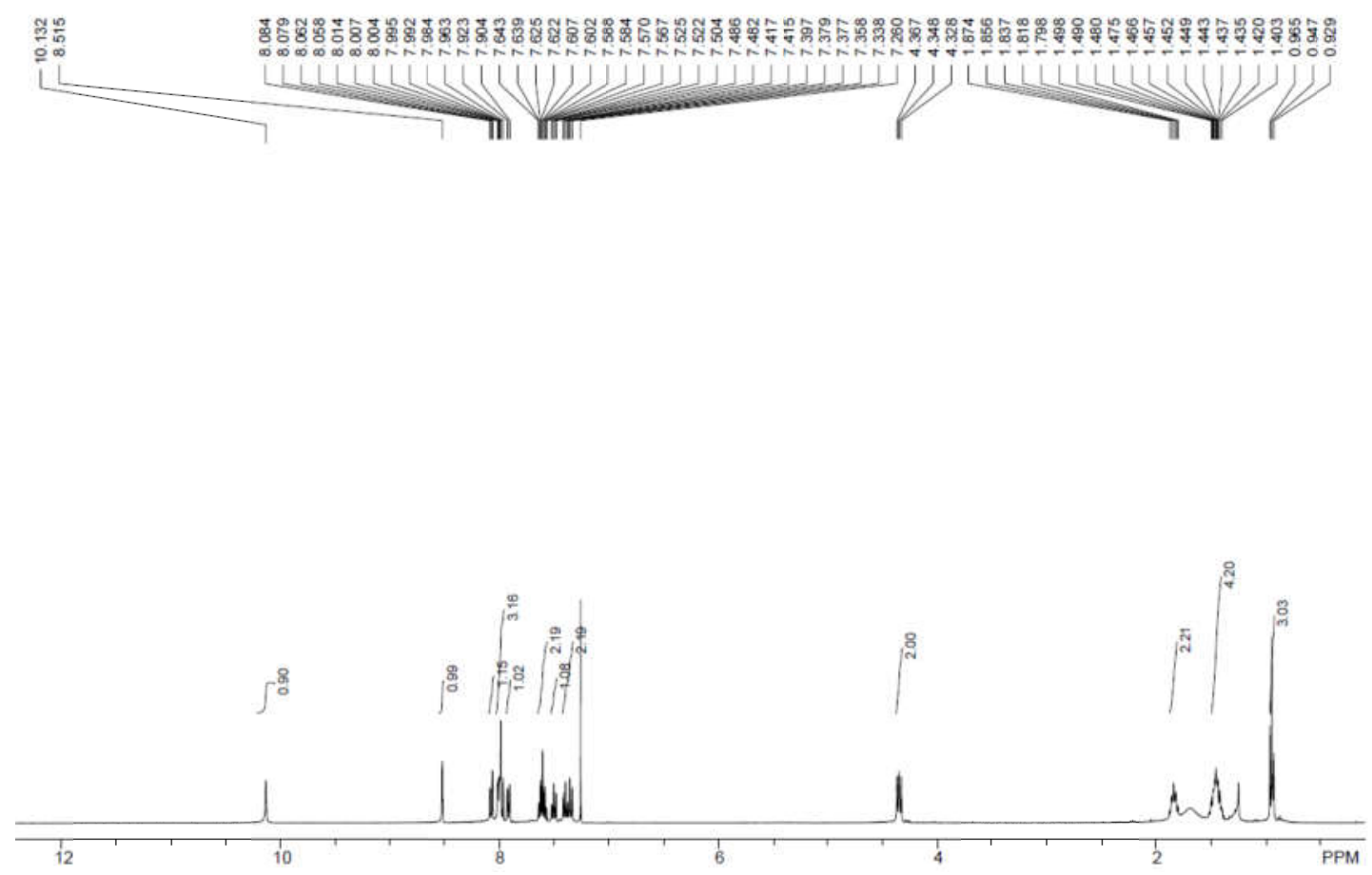

Figure S51 $\quad{ }^{1} \mathrm{H}$ spectra of $\mathbf{3 a j}$

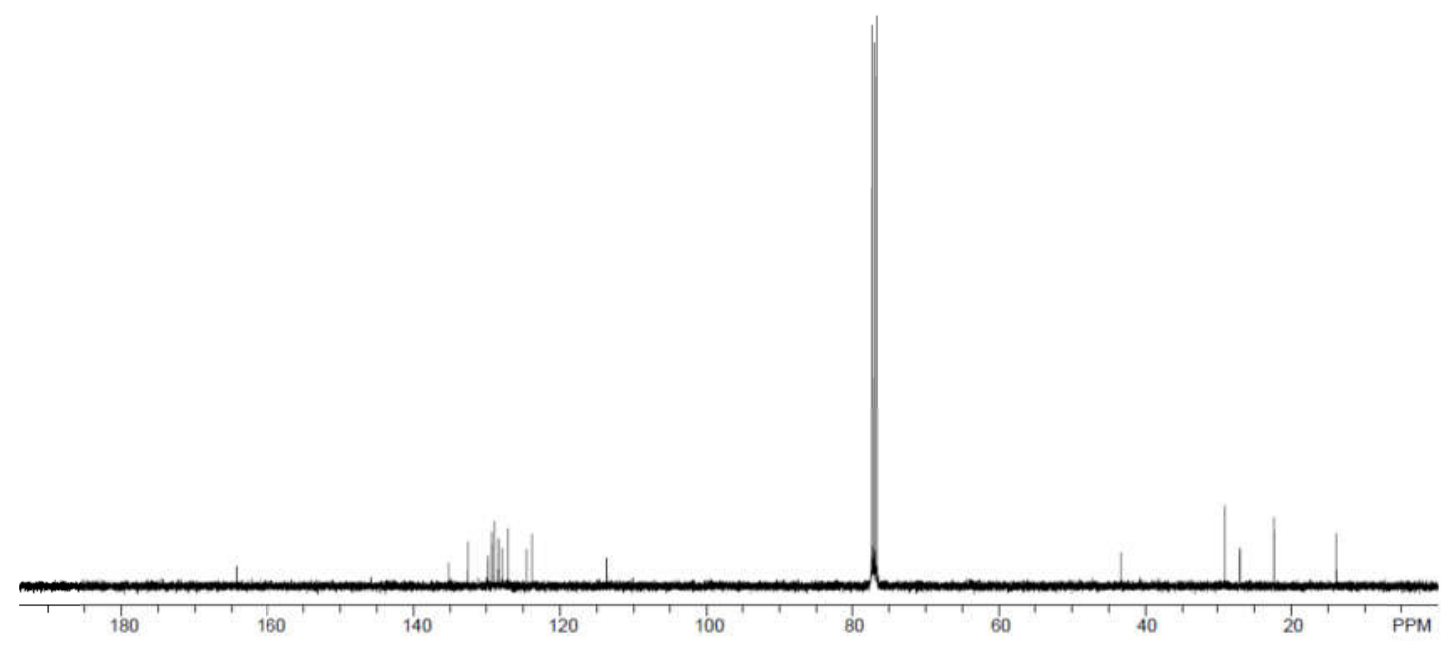

Figure S52 $\quad{ }^{1} \mathrm{H}$ spectra of $\mathbf{3 a j}$ 
$N$-(3-oxo-4-pentyl-3,4-dihydroquinoxalin-2-yl)thiophene-2-carboxamide (3ak)
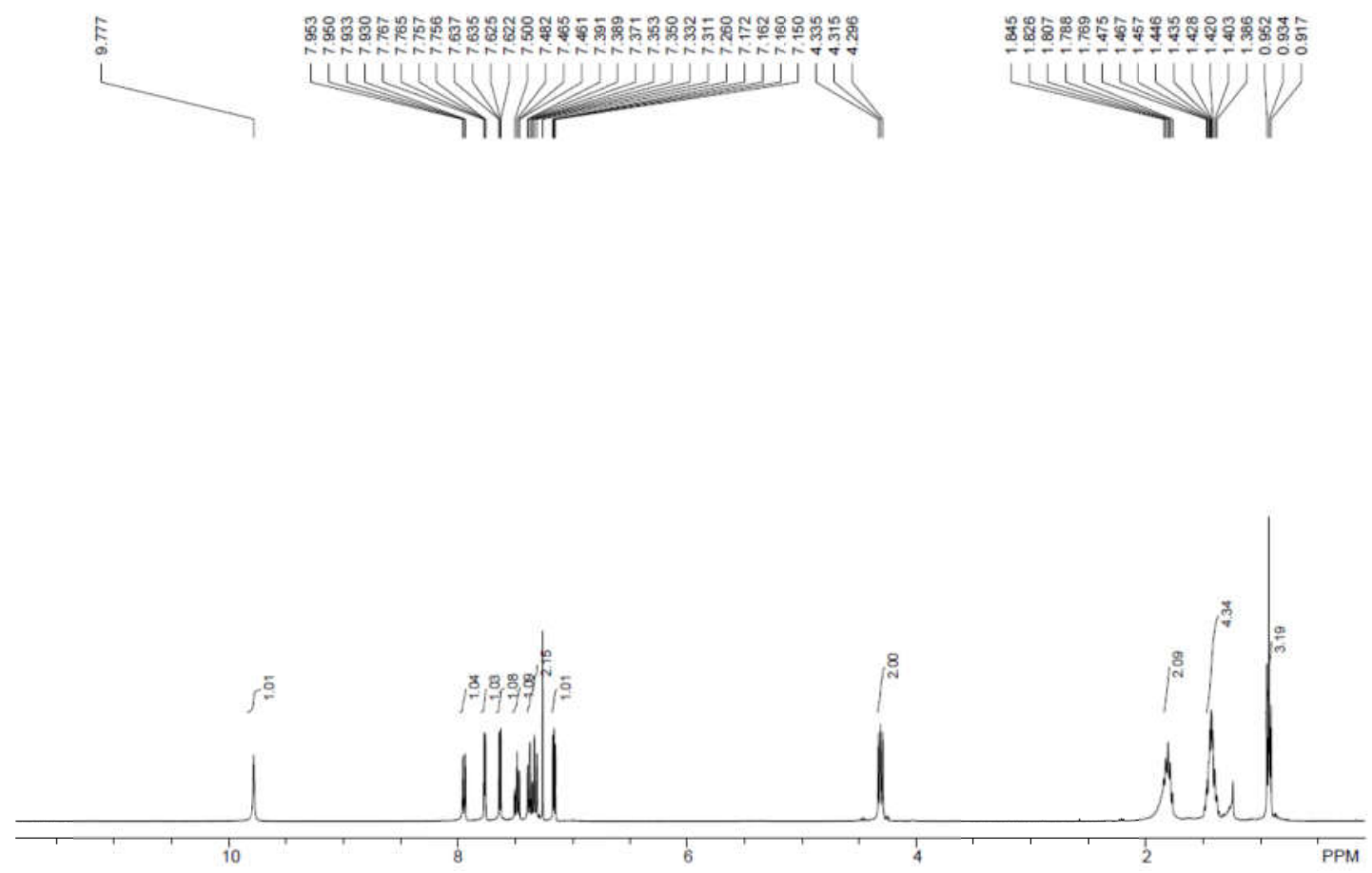

Figure S53 $\quad{ }^{1} \mathrm{H}$ spectra of $\mathbf{3 a k}$

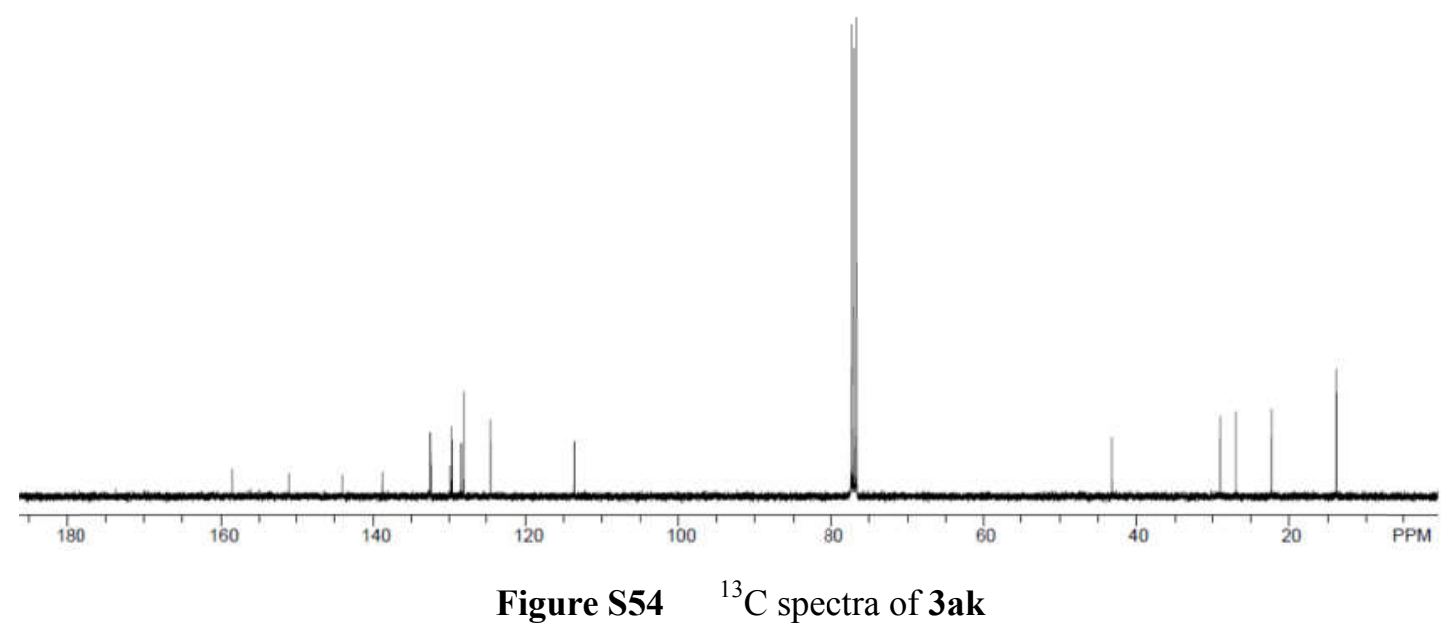


$N$-(3-oxo-4-pentyl-3,4-dihydroquinoxalin-2-yl)butyramide (3al)
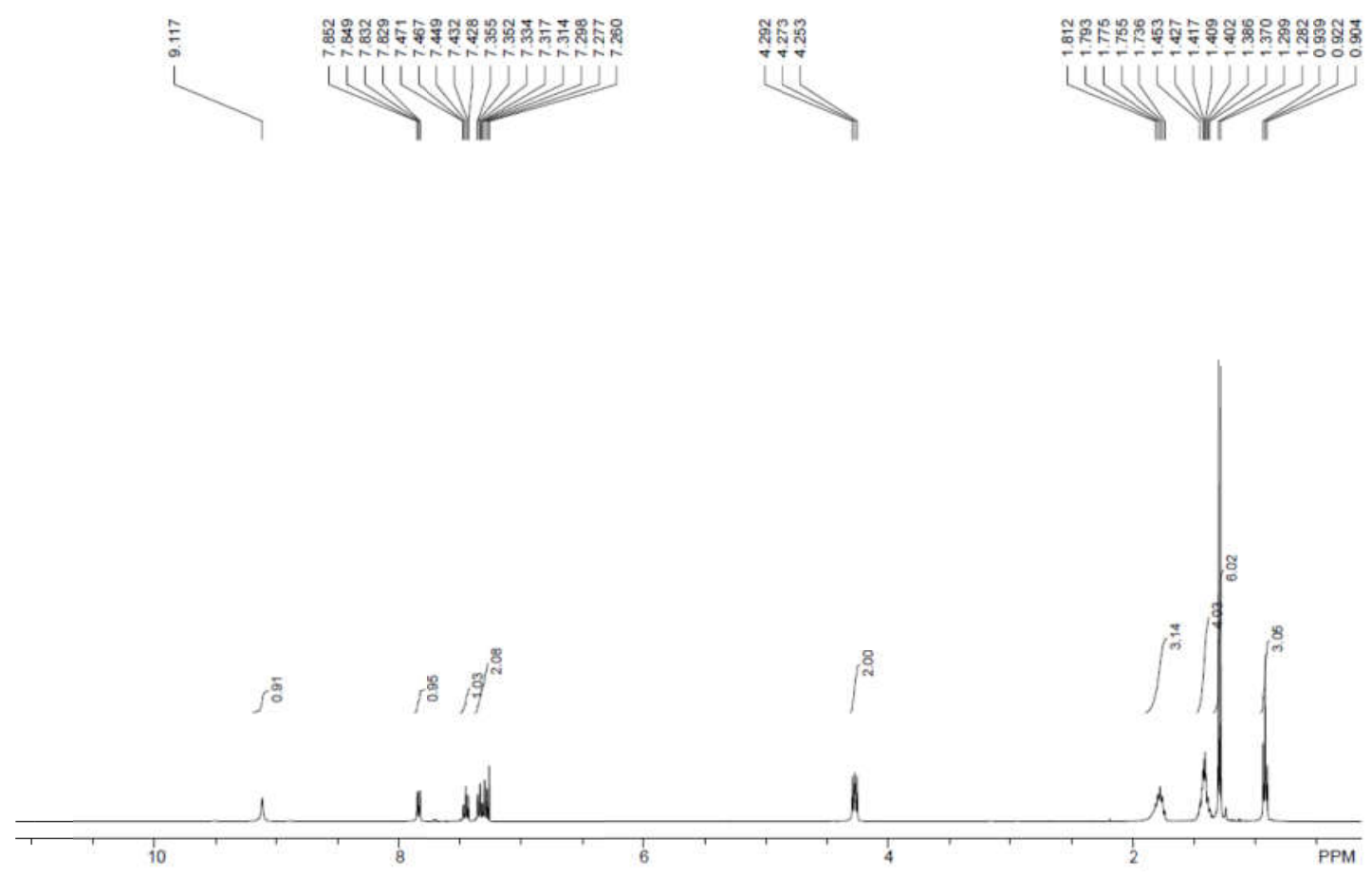

Figure $\mathbf{S 5 5} \quad{ }^{1} \mathrm{H}$ spectra of $\mathbf{3 a l}$

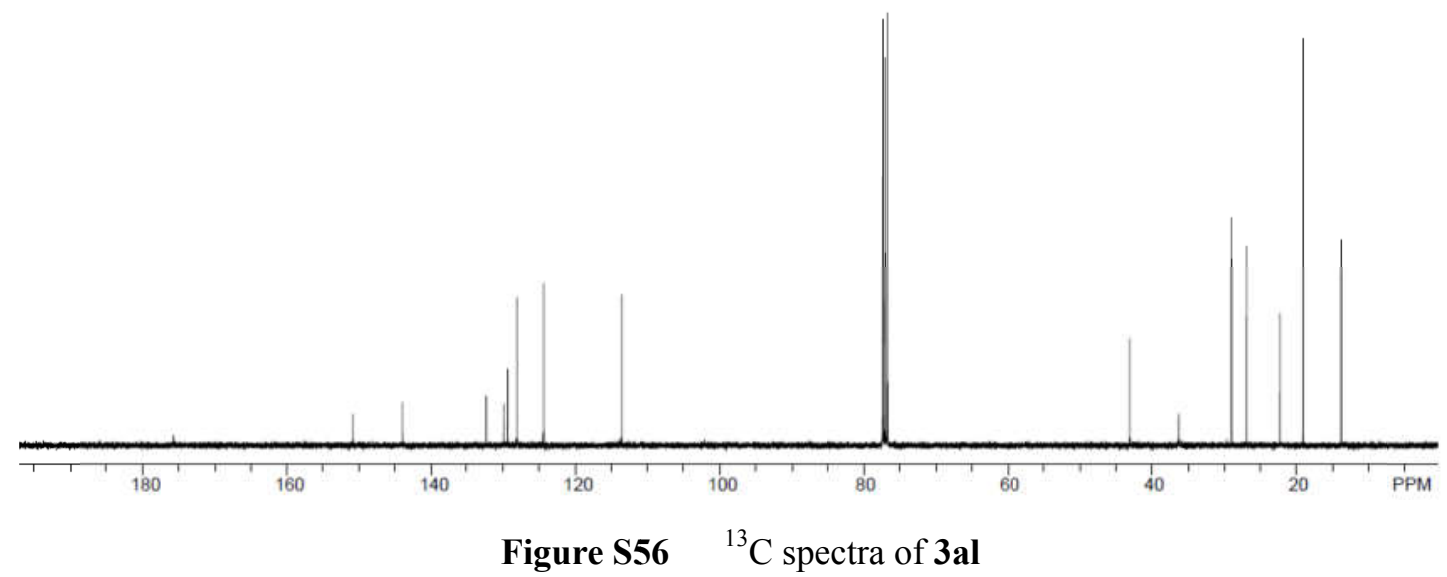


$N$-(3-oxo-4-pentyl-3,4-dihydroquinoxalin-2-yl)isobutyramide (3am)
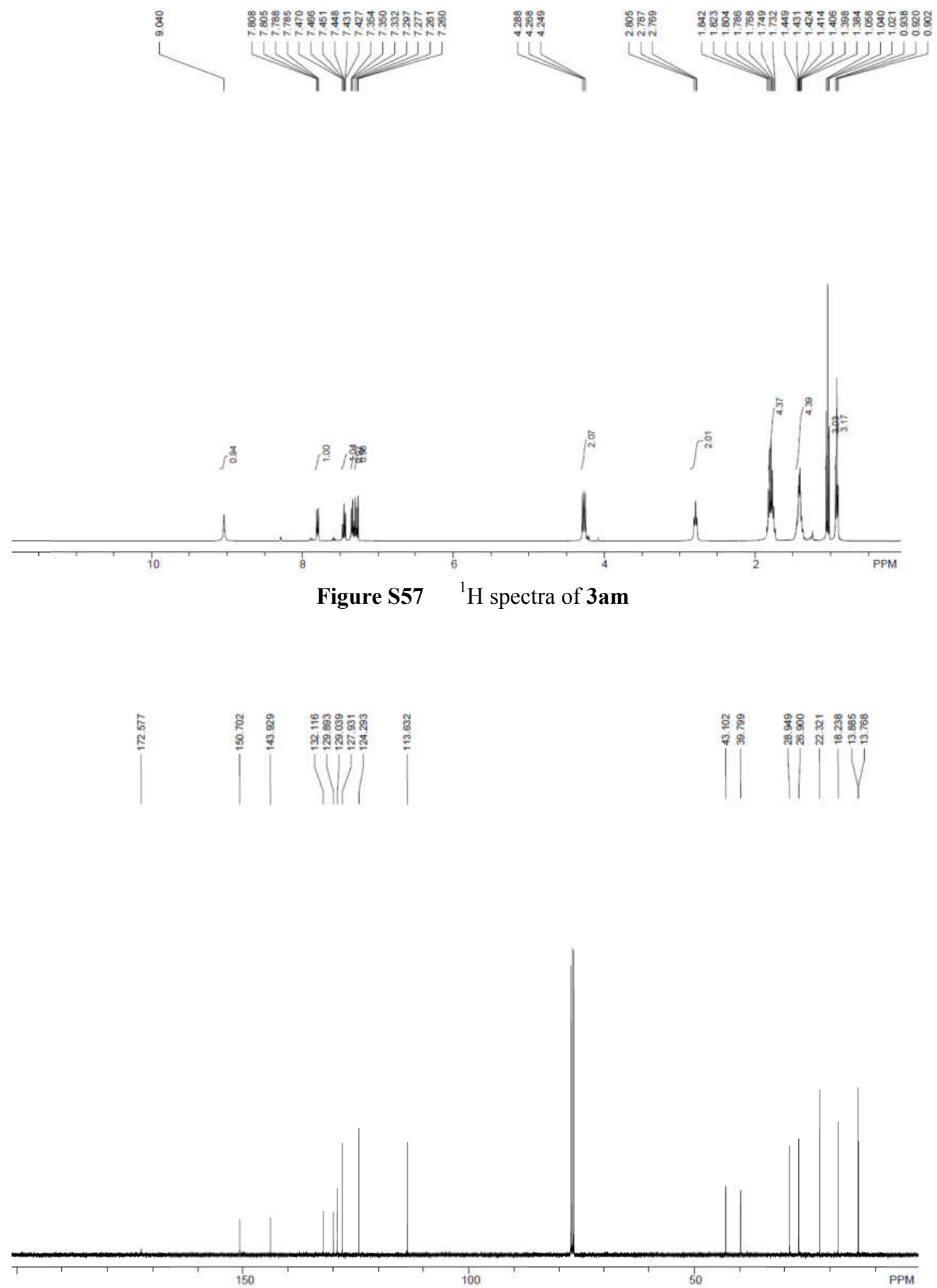

Figure S58 ${ }^{1} \mathrm{H}$ spectra of $\mathbf{3 a m}$ 
$\mathrm{N}$-(3-oxo-4-pentyl-3,4-dihydroquinoxalin-2-yl)pivalamide (3an)

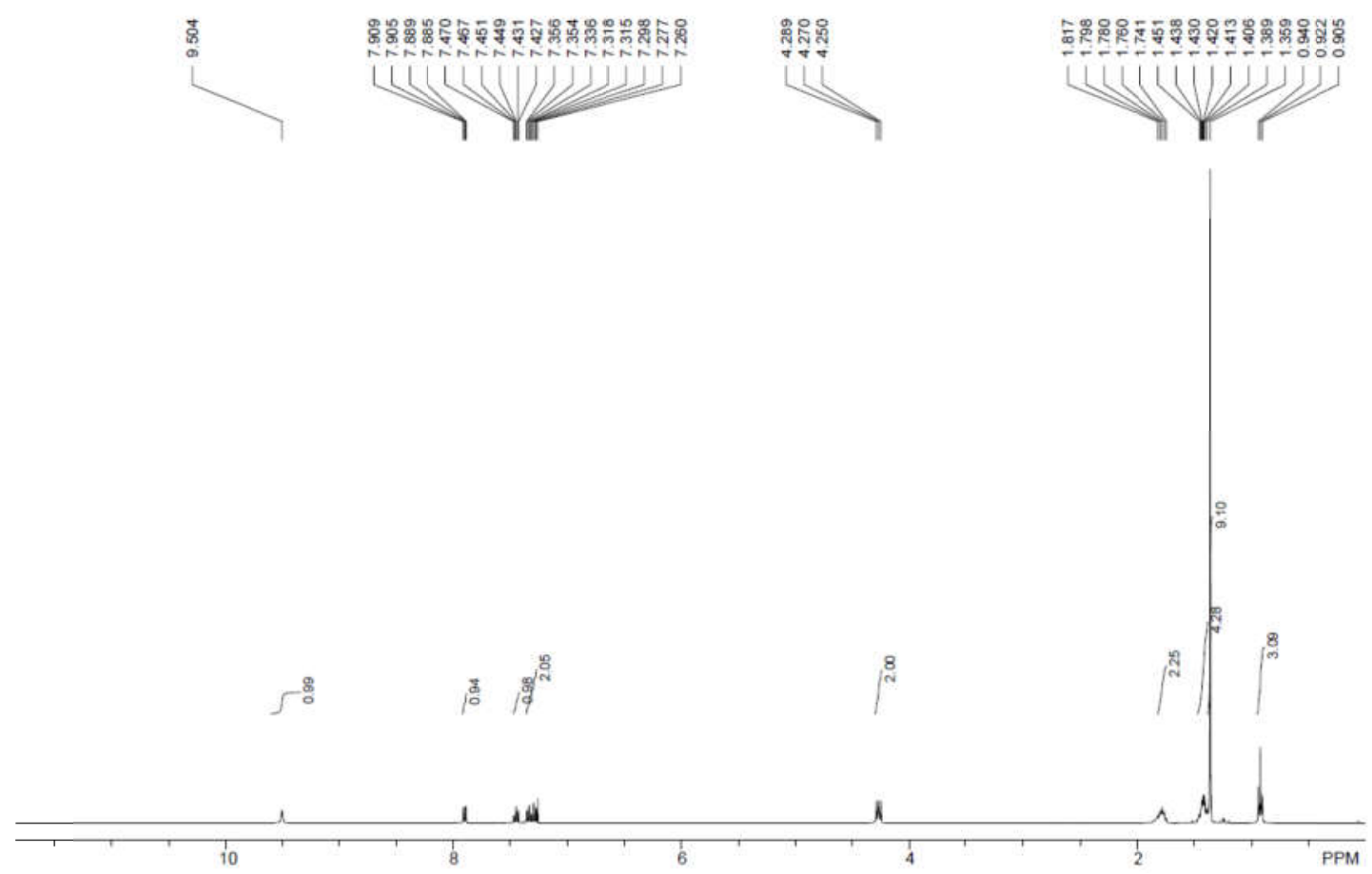

Figure S59 ${ }^{1} \mathrm{H}$ spectra of 3an

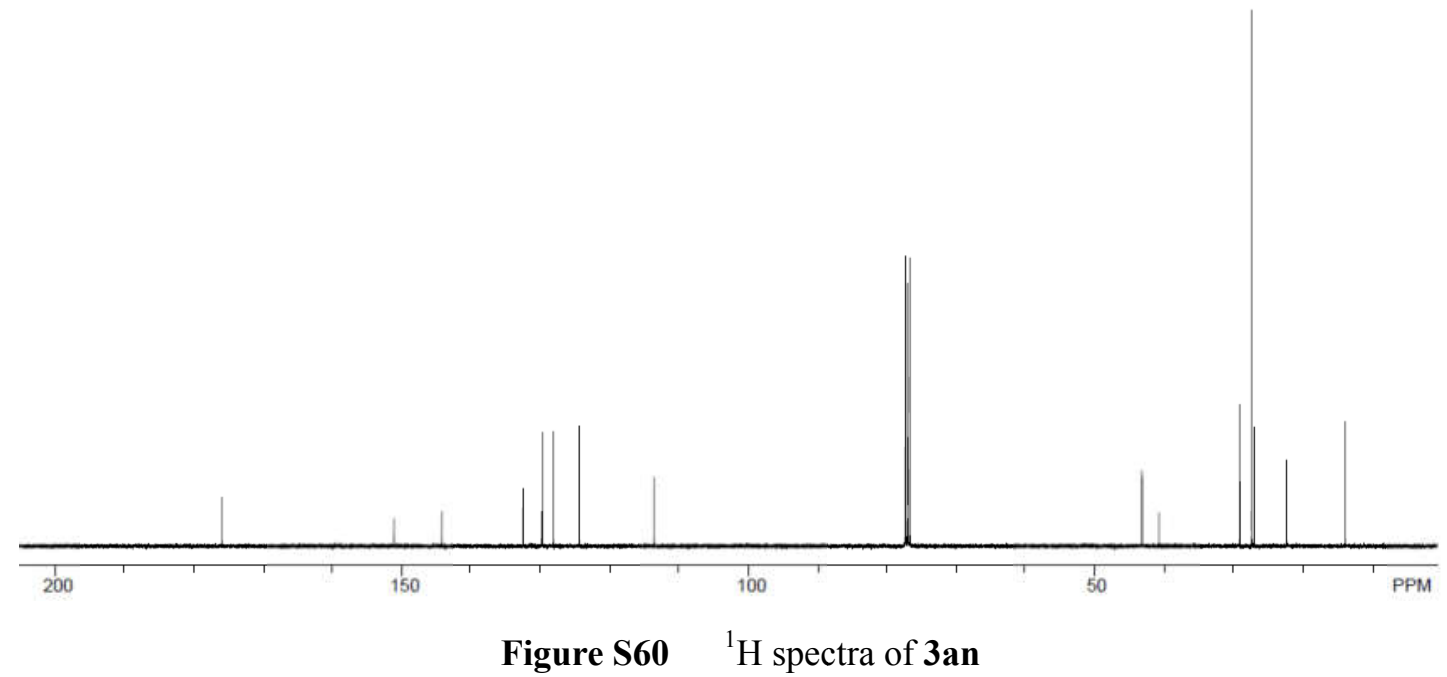


$\mathrm{N}$-(3-oxo-4-pentyl-3,4-dihydroquinoxalin-2-yl)cyclopropanecarboxamide (3ao)
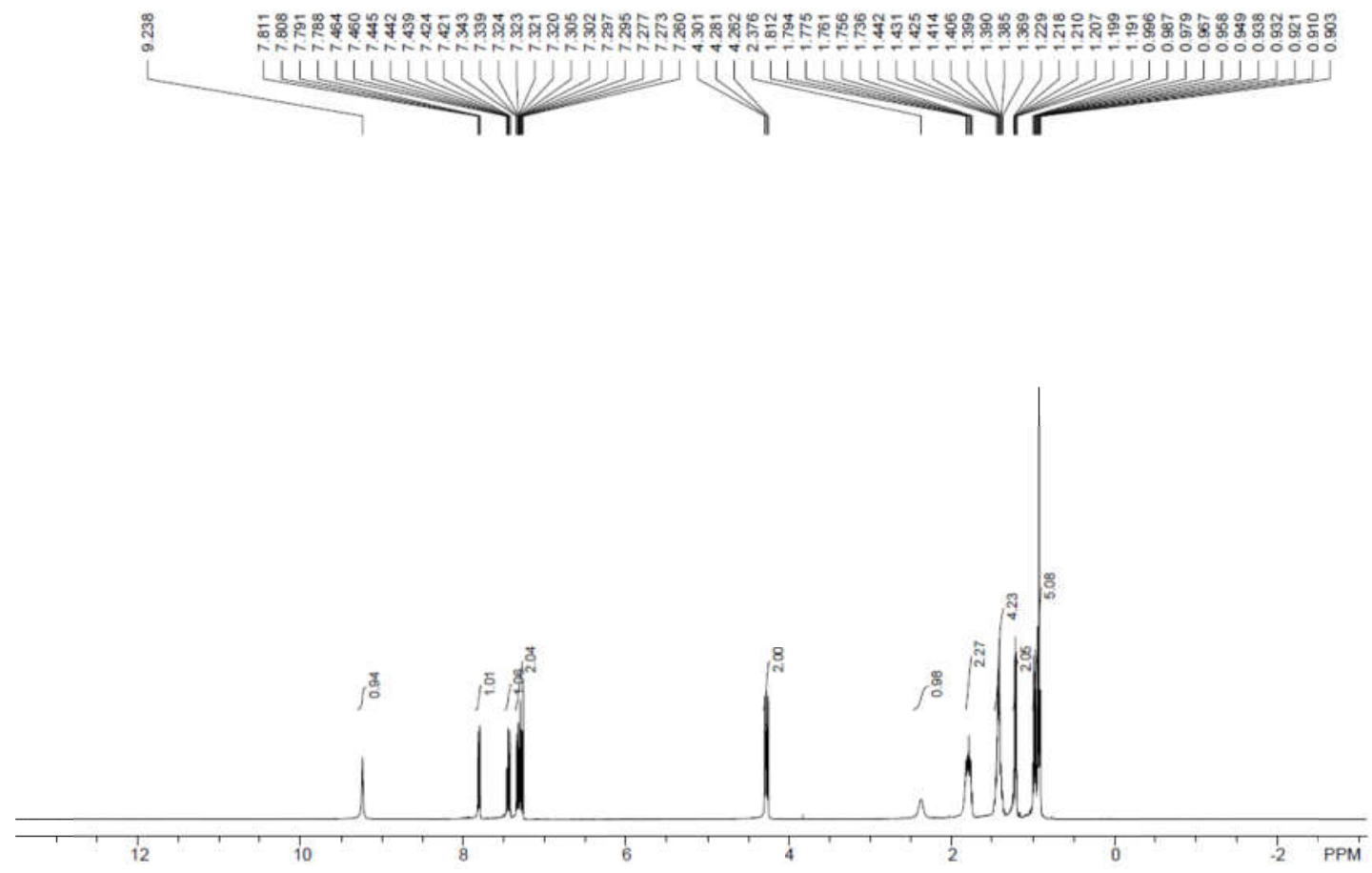

Figure S61 ${ }^{1} \mathrm{H}$ spectra of $\mathbf{3 a o}$

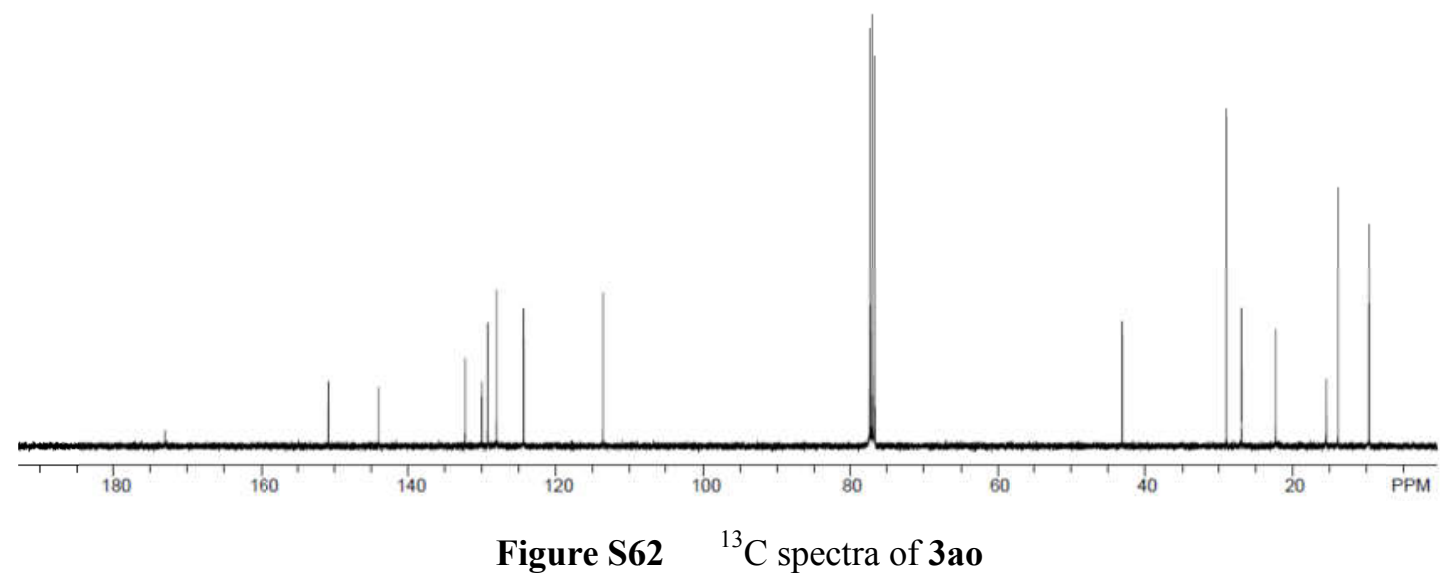


$\mathrm{N}$-(3-oxo-4-pentyl-3,4-dihydroquinoxalin-2-yl)cyclohexanecarboxamide (3ap)
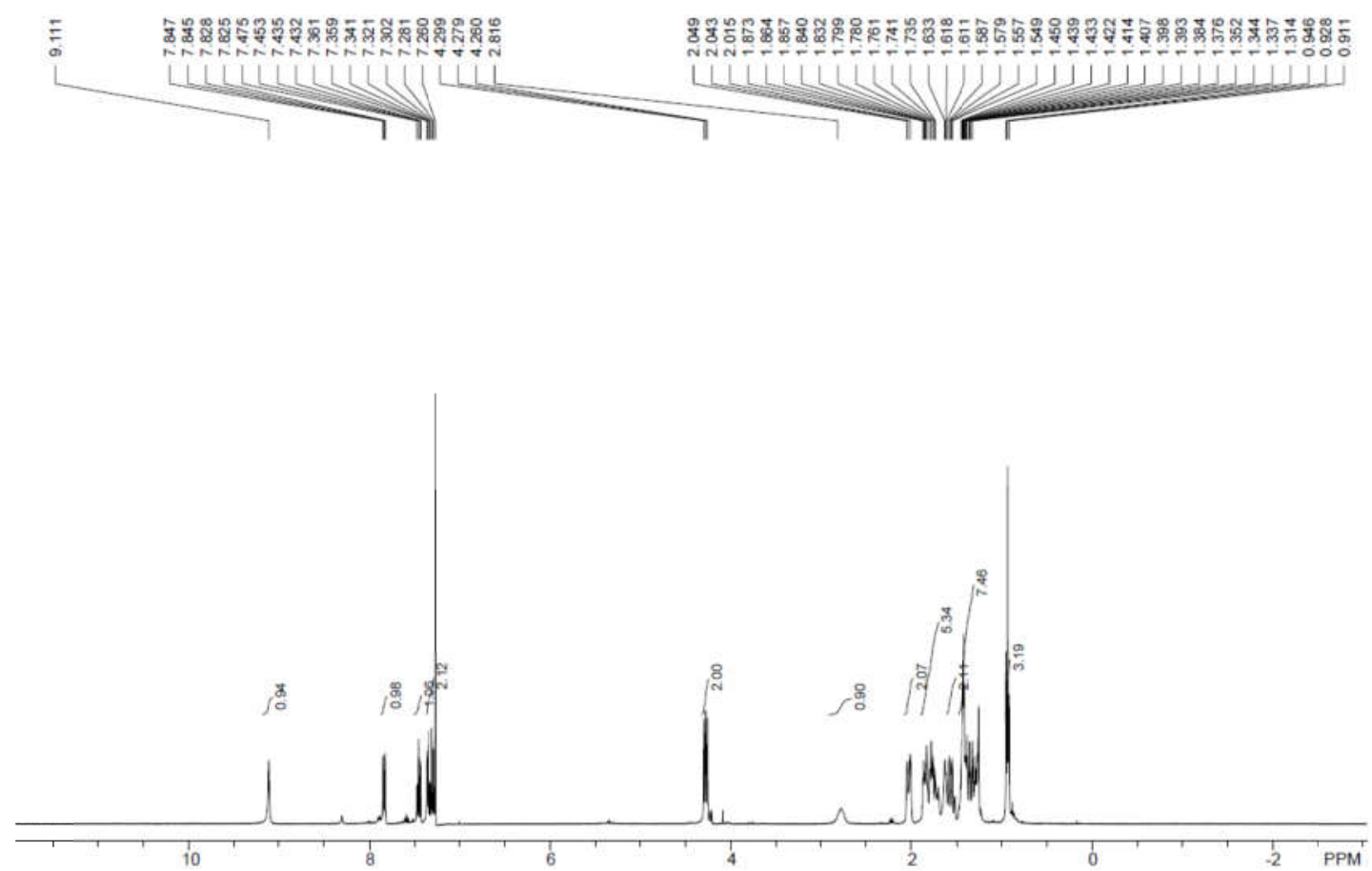

Figure S63 $\quad{ }^{1} \mathrm{H}$ spectra of 3ap

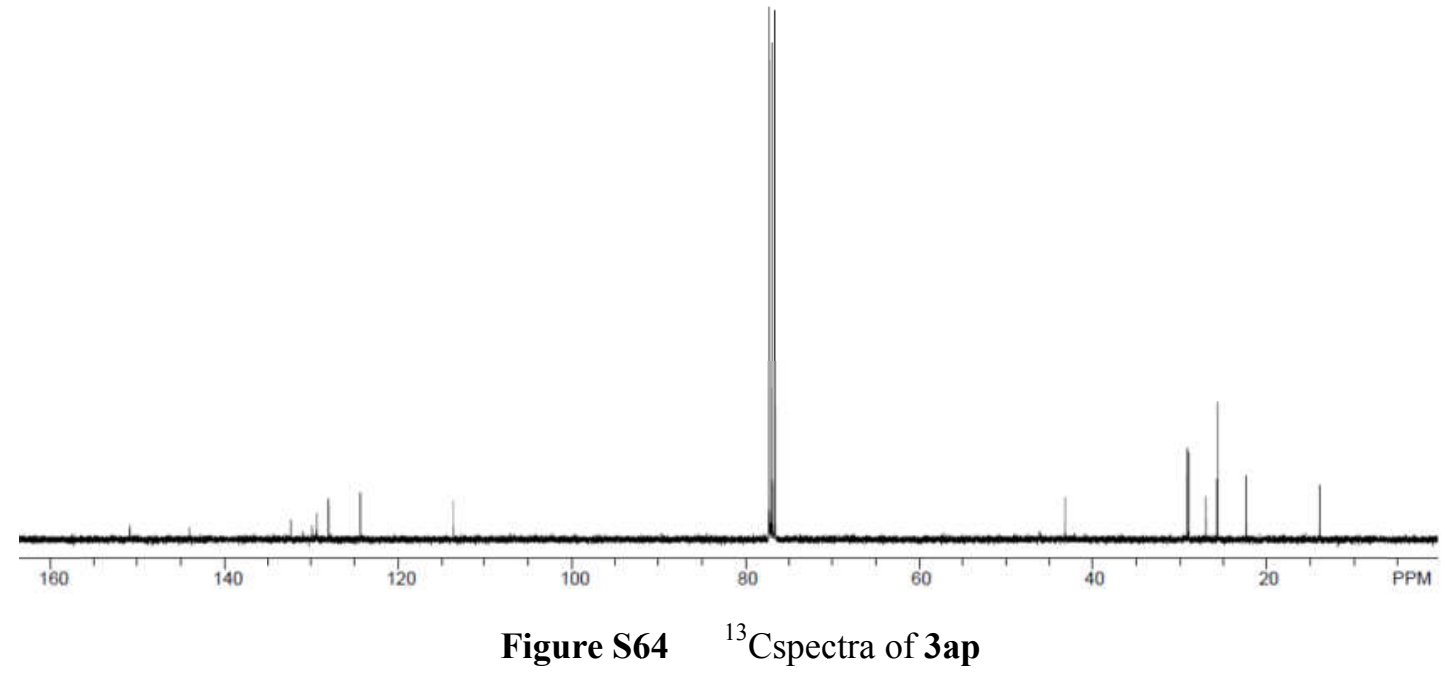

